\title{
Investigation of myelin membrane adhesion and compaction in the central nervous system
}

\author{
Dissertation \\ In partial fulfillment of the requirements for the degree \\ "Doctor rerum naturalium (Dr. rer. nat.)" \\ in the Molecular Medicine Study Program \\ at the Georg-August University Göttingen
}

submitted by

Mostafa Bakhti

born in

Dehloran, Iran

Göttingen 2012 
Members of the Thesis Committee:

Prof. Dr. Mikael Simons, Reviewer

Max Planck Institute of Experimental Medicine

Department of Neurology, University of Göttingen

Prof. Dr. Silvio Rizzoli, Reviewer

STED Microscopy of Synaptic Function, European Neuroscience Institute (ENI)

Dr. Judith Stegmüller, Ph.D.

Max Planck Institute of Experimental Medicine

Date of Disputation: 


\section{Affidavit}

Here I declare that my doctoral thesis entitled "Investigation of myelin membrane adhesion and compaction in the central nervous system" has been written independently with no other sources and aids than quoted.

Mostafa Bakhti

September, 2012

Göttingen, Germany 


\section{Related publications}

Mostafa Bakhti, Christine Winter and Mikael Simons. (2011) Inhibition of myelin membrane sheath formation by oligodendrocyte-derived exosome-like vesicles. J. Biol. Chem.; 286:787-796

Mostafa Bakhti, Nicolas Snaidero, David Schneider, Shweta Aggarwal, Wiebke Möbius, Andreas Janshoff, Matthias Eckhardt, Klaus Armin Nave and Mikael Simons. PLP adhesive property and sialic acid removal increase myelin adhesion and stability in the CNS.

Manuscript under preparation 


\section{Contents}

Contents vii

List of Figures $\ldots \ldots \ldots \ldots \ldots \ldots \ldots \ldots$ xi

List of Tables . . . . . . . . . . . . . . . . . . . . xiii

$\begin{array}{ll}\text { Abbreviations } & \text { Xv }\end{array}$

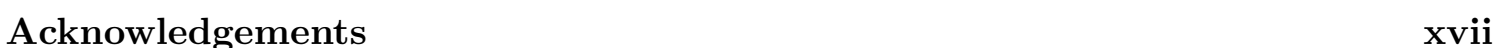

$\begin{array}{ll}\text { Abstract } & \text { xix }\end{array}$

1 Introduction 1

1.1 Central nervous system myelin . . . . . . . . . . . . . . . . 1

1.1.1 CNS myelin structure . . . . . . . . . . . . . . . 1

1.1 .2 Myelin composition . . . . . . . . . . . . . . . 3

1.1.2.1 Myelin proteins . . . . . . . . . . . . . 3

1.1.2.2 Myelin lipids . . . . . . . . . . . . . . . . . . . 4

1.1.3 Oligodendrocyte development . . . . . . . . . . . . . . . 6

1.1.4 Myelin biogenesis and assembly . . . . . . . . . . . . . . 9

1.1.5 Axon-glia communication . . . . . . . . . . . . . 9

1.1.6 Myelin pathology . . . . . . . . . . . . . . . . . . . 10

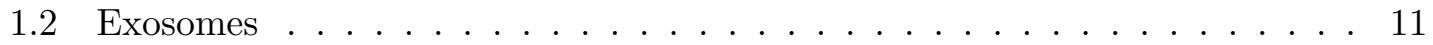

1.2.1 Composition of the exosomes . . . . . . . . . . . . 12

1.2.2 Formation and release of exosomes . . . . . . . . . . . . . . 12

1.2.3 Function of exosomes . . . . . . . . . . . . . . . . . . . . 14

1.3 Cell adhesion and glycocalyx $\ldots \ldots \ldots \ldots$

1.3 .1 Cell adhesion . . . . . . . . . . . . . . . . . 16

1.3 .2 Glycocalyx . . . . . . . . . . . . . . . . . 16

1.3.3 Glycocalyx involvment in cell adhesion . . . . . . . . . . . . . . . 17 
1.3.4 Sialic acid and its polymer PSA . . . . . . . . . . 18

1.4 Aims of the study . . . . . . . . . . . . . . . . . . . . 19

2 Materials and Methods $\quad 21$

2.1 Materials . . . . . . . . . . . . . . . . . . . . . . . . 21

2.1.1 Chemicals and consumables . . . . . . . . . . . . . 21

2.1.2 Antibodies and labeling components . . . . . . . . . . . . . . 22

2.1 .3 Commercial kits . . . . . . . . . . . . . . . . . . . . 23

2.1 .4 Animals . . . . . . . . . . . . . . . . . . . . 23

2.1.5 Plasmids . . . . . . . . . . . . . . . . . . . 24

2.1 .6 Media and sera . . . . . . . . . . . . . . . . . 24

2.1.6.1 Commercial media, sera and solutions . . . . . . . . . 24

2.1.6.2 Meida preparation . . . . . . . . . . . . . 25

2.1 .7 Softwares . . . . . . . . . . . . . . . . 25

2.2 Methods . . . . . . . . . . . . . . . . . . . 26

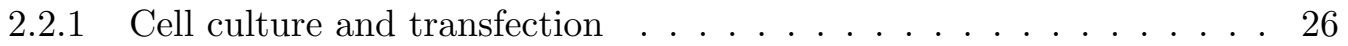

$2.2 .1 .1 \quad$ Primary culture . . . . . . . . . . . . 26

$2.2 .1 .2 \quad$ PtK2 cells . . . . . . . . . . . . . 26

2.2.1.3 Transfection . . . . . . . . . . . . . . 27

2.2.2 Exosomes Preparation . . . . . . . . . . . . . . . . . 27

2.2 .3 Proliferation assay . . . . . . . . . . . . . . . . . 27

2.2 .4 RhoA activity measurements . . . . . . . . . . . . . 28

2.2 .5 Protein biochemistry . . . . . . . . . . . . . . . . . 28

2.2.5.1 Preparation of cell lysates . . . . . . . . . . . . . 28

2.2.5.2 SDS-PAGE . . . . . . . . . . . . . . . 29

2.2.5.3 Western Blot . . . . . . . . . . . . . . . . 29

2.2.6 Myelin membrane analysis . . . . . . . . . . . . . . 30

2.2.6.1 Myelin preparation . . . . . . . . . . . . . 30

2.2.6.2 Myelin particles preparation . . . . . . . . . . 30

2.2.6.3 Myelin particles labelling and binding assay . . . . . . . . . 30

2.2 .7 Lectin staining . . . . . . . . . . . . . . . . . . . . 31

2.2 .8 Neuraminidase (sialidase) treatment . . . . . . . . . . . . 31

2.2.9 Click-iT glycoprotein labeling and detection . . . . . . . . . . . . 32

2.2.10 Exogenous gangliosides incorporation . . . . . . . . . . . . . 32 
2.2 .11 Lipid analysis . . . . . . . . . . . . . . . . . . . . 32

2.2.11.1 Lipid extraction . . . . . . . . . . . . . 32

2.2.11.2 Liposome preparation and aggregation . . . . . . . . 33

2.2 .11 .3 Dot blot . . . . . . . . . . . . . . . . . . 33

2.2 .12 Immunocytochemistry and imaging . . . . . . . . . . . . 33

2.2.12.1 Immunocytochemistry . . . . . . . . . . . . . . . 33

2.2.12.2 Confocal Microscopy . . . . . . . . . . . . . . . . 34

2.2.12.3 Image Analysis . . . . . . . . . . . . . . . . . . . . . . 34

2.2.13 Atomic Force Microscopy (Single particle force spectroscopy) . . . . 34

2.2 .14 Electron microscopy . . . . . . . . . . . . . . . . . 35

2.2.14.1 Conventional embedding . . . . . . . . . . 35

2.2.14.2 High pressure freezing . . . . . . . . . . . 35

2.2.14.3 Freeze substitution and embedding . . . . . . . . . 35

2.2.14.4 Sectioning and electron microscopy . . . . . . . . . . 36

$\begin{array}{llr}3 & \text { Results } & 37\end{array}$

3.1 The function of oligodendrocyte-derived exosome-like vesicles in the CNS . 37

3.1.1 Characterization of oligodendrocyte-derived exosomes . . . . . . 37

3.1.2 The effect of exosomes on myelination . . . . . . . . . . . . . 38

3.1.3 Effect of neuronal signals on exosome release by primary oligodendrocytes . . . . . . . . . . . . . . . . . 4 40

3.1.4 Effect of OL-derived exosomes on oligodendrocytes . . . . . . . 440

3.1.5 RhoA pathway involvement in exosome effect on OLs . . . . . . . 42

3.1.6 Fyn and FAK phosphorylation are involved in exosomes effect on OLs 44

3.2 Mechanism of myelin membrane adhesion and compaction in CNS . . . . 48

3.2.1 A new assay to assess myelin membrane adhesion in vitro . . . . . . 48

3.2.2 The preparation and characterization of myelin particles . . . . . . . 49

3.2.3 Myelin particles preferentially bind to oligodendrocyte membrane

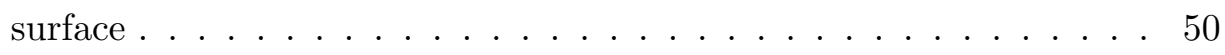

3.2.4 PLP plays a role in the interaction of myelin particles with OLs . . 51

3.2.5 PLP increases physical stability of myelin . . . . . . . . . . . 53

3.2.6 Reduction in glycocalyx during oligodendrocytes maturation correlates with an increased in membrane adhesiveness . . . . . . . . 59 
3.2.7 Reduction of sialic acid from myelin-like membrane increases OLs affinity towards myelin particles . . . . . . . . . . . . . 64

3.2.8 Negative surface charges interfere with myelin membrane adhesiveness 67

3.2.9 Partially glycocalyx reconstitution reduces particle-cell interaction . 69

3.2.10 PLP achieves its function upon sialic acid removal . . . . . . . . 72

4 Discussion $\quad 75$

4.1 The function of oligodendrocyte exosomes in the CNS . . . . . . . . . 75

4.1.1 Regulation of oligodendrocyte development . . . . . . . . . 75

4.1.2 Exosomes secreted by oligodendrocytes inhibit myelination process . 76

4.1.3 Exosomes inhibit terminal differentiation of OLs to become myeli-

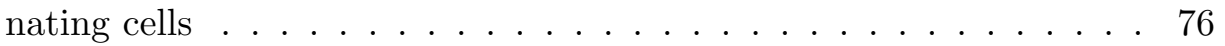

4.1.4 Exosomes influence OLs through a common signalling pathway triggering cell retraction . . . . . . . . . . . . . . 77

4.1.5 Exosomes and myelin debris have the same effect on OLs . . . . . 78

4.2 Myelin adhesion and compaction in central nervous system . . . . . . . . 79

4.2.1 Myelin compaction and a new model system for its assessment . . . 79

4.2.2 The role of PLP in myelin adhesion and compaction . . . . . . . . 79

4.2.3 The involvement of PLP in supporting the physical integrity of CNS myelin . . . . . . . . . . . . . . . . . 80

4.2.4 Oligodendrocyte glycocalyx is reduced during their maturation . . . 82

4.2.5 The mechanisms involved in glycocalyx removal from OLs surface . 82

4.2.6 Down-regulation of sialic acid content is necessary for myelin com-

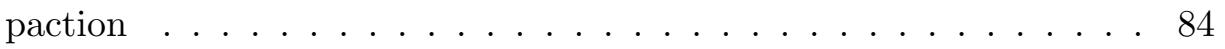

5 Summary and conclusion $\quad 85$

$\begin{array}{lr}\text { Bibliography } & 89\end{array}$

$\begin{array}{ll}\text { Curriculum Vitae } & 107\end{array}$ 


\section{List of Figures}

1.1 The structure of CNS myelin . . . . . . . . . . . . . . 2

1.2 The organization of myelin compact area . . . . . . . . . . 5

1.3 Oligodendrocyte development . . . . . . . . . . . . . . . 8

1.4 Exosome composition and formation . . . . . . . . . . . . . . 13

$2.1 \quad$ Myelin particle preparation and labeling . . . . . . . . . . . . . 31

3.1 Characterization of oligodendrocyte-derived exosomes _ . . . . . . . 38

3.2 Electron microscopic of oligodendrocyte-derived exosomes . . . . . . . . . 39

3.3 Exosomes inhibit myelination . . . . . . . . . . . . . . . 39

3.4 Neuronal conditioned medium reduces exosome release by OLs . . . . . . 40

3.5 Exosome effects on the proliferation, viability, and differentiation of oligodendrocytes . . . . . . . . . . . . . . . . . . . 4 4 41

3.6 Exosomes inhibit cell surface expansion of oligodendrocytes . . . . . . . 42

$3.7 \quad$ Exosomes do not influence the mTOR pathway . . . . . . . . . . . . . . 43

3.8 Inhibition of ROCK prevents the effect of exosome on cell surface size . . 44

3.9 Inhibition of myosin-II reduces the effect of exosome on cell surface size . 45

3.10 Inhibition of actomyosin contractility prevents the effect of exosomes on oligodendrocyte cell surface area. . . . . . . . . . . . . . 46

3.11 NCM increases cell surface area by activation of Src proteins . . . . . . 47

3.12 Exosomes affect phosphorylation of Fyn protein . . . . . . . . . . . . 47

3.13 Exosomes inhibit autophosphorylation of Focal adhesion kinase (FAK) . . 48

3.14 Binding assay . . . . . . . . . . . . . . . . . . . . . . . 49

3.15 Preparation and characterization of myelin particles . . . . . . . 50

3.16 Immunolabeling of myelin particles . . . . . . . . . . . . . . . . 51

3.17 Interaction of myelin particles with different glial cells . . . . . . . . . 53

3.18 Myelin particles interact with the myelin-like membrane surface . . . . . . 54

3.19 PLP increases particle-cell interaction . . . . . . . . . . . . . 55 
3.20 Presence of PLP in one side of interacting membranes, increases the binding of particles to oligodendrocytes . . . . . . . . . . . . 56

3.21 PLP increases the interaction between myelin membrane particles . . . . . 57

3.22 PLP increases aggregation of myelin-derived liposomes . . . . . . . . . . 58

3.23 PLP increases the size of myelin particles _ . . . . . . . . . . . . . . 59

3.24 Single particle force spectroscopy reveals stronger adhesion between particles and cells in the presence of PLP . . . . . . . . . . . . . 60

3.25 The stability of myelin is reduced in the absence of PLP . . . . . . . . . 61

3.26 A reduction in the surface glycocalyx occurs during oligodendrocytes maturation . . . . . . . . . . . . . . . . . . . . 62

3.27 Myelin-like membrane adhesiveness is increased during oligodendrocytes

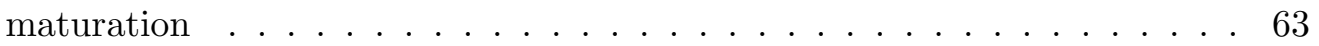

3.28 Sialic acid content is reduced during oligodendrocytes maturation . . . . . 65

3.29 Myelinated area possess low sialic acid content . . . . . . . . . . . . 66

3.30 Enzymatic removal of sialic acid enhances the interaction between oligodendrocyte surface with myelin particles . . . . . . . . . . . . 68

3.31 Cell surface negative charges reduce myelin particle interaction with oligodendrocytes . . . . . . . . . . . . . . . . . . . 69

3.32 Partially reconstitution of glycocalyx into myelin-like membranes decreases their interaction with myelin particles _ . . . . . . . . . 70

3.33 Exogenous administration of gangliosides reduces adhesiveness of myelinlike membrane . . . . . . . . . . . . . . . . . . . . . . 71

3.34 Polysialyl-transferase (ST8SiaIV) is down-regulated during OL maturation 72

3.35 PSA-expressing oligodendrocytes produce less stable myelin . . . . . . . . 73

3.36 PSA expression reduces the interaction between myelin particles . . . . . 73

3.37 PLP expression and sialic acid removal triggers myelin particle binding to non-myelin membranes . . . . . . . . . . . . . . . . . . . 74

5.1 A model for the function of oligodendrocyte-derived exosomes . . . . . . . 86

5.2 A model for myelin adhesion and compaction in CNS . . . . . . . . . . 87 


\section{List of Tables}

2.1 Inhibitors and chemical components . . . . . . . . . . . . . . 21

2.2 Primary antibodies . . . . . . . . . . . . . . . . . . 22

2.3 Secondary antibodies and fluorophore-coupled compounds . . . . . . . . 23

$2.4 \quad$ Commercial kits . . . . . . . . . . . . . . . . . . . . . . . . . 23

2.5 Mouse and rat strains . . . . . . . . . . . . . . . . . . . 24

2.6 Commercial media, sera and solutions . . . . . . . . . . . . . . 24

$2.7 \quad$ Softwares . . . . . . . . . . . . . . . . . . . 25 


\title{
Abbreviations
}

\author{
APC Antigen presenting cell \\ APS Ammonium persulfate \\ CGT UDP-galactose:ceramide galactosyltransferase \\ CNPase 2',3'-cyclic nucleotide 3'-phosphodiesterase \\ CNS Central nervous system \\ CST Cerebroside sulfotransferase \\ ECM Extracellular matrix \\ EDTA Ethylenediaminetetraacetic acid \\ ESCRT Endosomal sorting complex required for transprort \\ GalC Galactosylceramide \\ GDP Guanosine diphosphate \\ GFAP Glial fibrillary acidic protein \\ GSLs Glycosphingolipids \\ GTP Guanosine triphosphate \\ GTPase Guanine triphosphatase (s) \\ HEPES 4-(2-hydroxyethyl)-1-piperazineethanesulfonic acid \\ hr Hour(s) \\ HRP Horse-radish peroxidase \\ IPL Intraperiod line \\ MAG Myelin-associated glycoprotein \\ MAL Myelin and lymphocyte protein \\ MBP Myelin basic protein \\ MDL Major dense line \\ MHC Major histocompatibility complex \\ $\min \quad$ Minute(s) \\ MOG Myelin oligodendrocyte glycoprotein
}


MS Multiple sclerosis

MVB Multivesicular body

NCAM Neural cell adhesion molecule

NCM Neuronal conditioned medium

OL Oligodendrocyte

OPC Oligodendrocyte precursor cells

OMgp Oligodendrocyte myelin glycoprotein

OSP Oligodendrocyte-specific protein

PAGE Polyacrylamide gel electrophoresis

PBS Phosphate buffered saline

PDGFR Platelet derived growth factor receptor

PLL Poly-L-lysine

PLP Proteolipid protein

PNS Peripheral nervous system

PSA Polysialic acid

PST Polysialyltransferase

Rab Ras-like protein in brain

ROCK Rho-associated protein kinase

RT Room temperature

SDS Sodium dodecyl sulphate

SNARE Soluble NSF attachment protein receptor

TEMED N,N,N',N'-Tetramethylethylene-diamine

WGA Wheat germ agglutinin 


\section{Acknowledgements}

I deeply thank my scientific advisor, Prof. Mikael Simons, who initiated and continuously supported the projects. I am grateful for his scientific guidance and technical advisory, which helped me to explore the fields related to this study. I am also grateful to my thesis committee members Prof. Silvio Rizzoli and Dr Judith Stegmüller for their comments and inspiring discussion during the meetings.

I sincerely thank the collaborators who participated in these studies: Nicolas Snaidero for electron microscopic analysis with the help of Wiebke Möbius; David Schneider and Prof. Andreas Janshoff for the performing single particle force spectroscopy; Christine Winter who contributed to the first related publication of the project; Prof. Klaus Armin Nave and Prof. Matthias Eckhardt for providing KPLP and PLP-PST mutant mice, respectively; Shweta Aggarwal for providing different versions of MAG construct and Shima Safaiyan for providing brain sections. I would like to thank Giselheid Schulz and Kirsten Fladung for their technical support. I would also like to acknowledge my colleagues at the Max Planck institutes for experimental medicine for help with equipment, reagents, and providing animals. I want to thank the coordination office of Molecular Medicine Program: Dr. Erik Meskauskas and Dr. Werner Albig.

I am grateful to all colleges and friends in AG Simons lab for their help and support and providing a friendly scientific environment during my PhD thesis: Natalia ManriqueHoyos, Shweta Aggarwal, Chieh Hsu, Aniket Ghosh, Nicolas Snaidero, Sebastian Schmitt, Mareike Schnaars, Katrin Strauss, Holger Budde, Larisa Yurlova, Olena Steshenko, Dirk Fitzner, Xingwen Sun, Tina Kling, Shima Safaiyan, Christina Reetz, Marie-Theres Weil, Schanila Nawaz, Nils Halbsgut, Lauren Haag, Alexander Herholt, Johannes Zimmermann, and karla Hans. 
I would like to thank Rohollah Babaei, Mehdi Pirouz, Ali Shahmoradi, Zohreh Farsi, Atefeh Pooryasin, Tahereh Ajam, Farnaz Shamsi, Mayur Vadhvani, Ahmad Mirkhani and many, many friends who supported and accompanied me during staying in Göttingen.

Finally my special thanks go to my family, especially my parents for their unlimited support and love and for everything that they have done for me. 


\section{Abstract}

Myelin is a multi-layered membrane which enwraps the axons in peripheral (PNS) and central nervous system (CNS). The formation and assembly of this structure is a multistep process that is regulated by a variety of extracellular factors. In the CNS, myelin is produced by oligodendrocytes. During development, the progenitors of these cells differentiate into mature oligodendrocytes that start to enwrap axons by myelin membrane sheaths after receiving the appropriate signal(s) from the microenvironment. However, the responsible signals to initiate this process are unknown. Here, we showed that oligodendrocytes release small microvesicles, exosomes, into the extracellular space that prevent the terminal differentiation of oligodendrocytes and subsequently myelin formation. These inhibitory effects were revealed to be mediated by activity of the RhoA-ROCK signalling cascade. Importantly, the exosome release by oligodendrocytes was significantly reduced when cells were incubated with the conditioned medium from neurons. Our results suggest that exosomes produced by oligodendrocytes maintain the cells in pre-myelinating stage, whereas in the presence of neuronal signals, exosomes secretion by oligodendrocytes is reduced and the autoinhibitory signals are relieved. Thus neurons may regulate the formation and release of oligodendroglial-derived exosomes in order to coordinate myelin membrane biogenesis and assembly.

In the second part of the thesis, the question of how myelin compaction is mediated was addressed. Whereas MBP is known to organize the interaction between myelin membranes from cytoplasmic side, the molecular mechanisms underlying the interaction between the outer leaflets still remain unclear. In general, the interaction between two opposite membranes requires the expression of adhesion molecules and the removal of repulsive components. Therefore, we investigated the role of proteolipid protein (PLP), as a putative adhesive molecule, and the glycocalyx, as a repulsive structure, during myelin compaction in the CNS. We analyzed the adhesion of purified myelin particles with the primary oligo- 
dendrocytes in order to mimic the interaction between myelin layers. Using this system we showed that PLP increases the adhesiveness of myelin membrane. We also found that PLP enhances physical stability of myelin using single particle force spectrometry. In addition, we observed a significant reduction in the glycocalyx during oligodendrocyte maturation which correlated with an increase in their surface affinity towards myelin particles. Further analysis indicated that the negative charge of sugar moieties, mainly sialic acid, is responsible for the reduction in myelin adhesiveness. Therefore, we propose that the adhesive properties of PLP along with the reduction of the glycocalyx, orchestrate myelin membrane adhesion and compaction in the CNS. 


\section{Chapter 1}

\section{Introduction}

\subsection{Central nervous system myelin}

Myelin consists of a multi-layered stack of membranes which spirally enwraps axonal segments in the peripheral (PNS) and central (CNS) nervous system. Schwann cells and oligodendrocytes (OLs) are responsible for myelination in PNS and CNS, respectively. Although myelin has an identical function, the cell biology of its biogenesis by myelinating glia is different in the PNS and CNS (Richardson et al., 2006; Chong and Chan, 2010). Myelin is important as an axonal insulator, which is essential for generating rapid saltatory impulse propagation of neuronal signal. In addition, this structure supports axonal survival and long term integrity by providing trophic factors (Nave and Trapp, 2008; Emery, 2010a). The importance of myelin is evident in pathological conditions such as multiple sclerosis (MS) in which this structure undergoes severe distortion (Franklin and Kotter, 2008).

\subsubsection{CNS myelin structure}

In the CNS, myelin biogenesis starts by the branching of oligodendrocytes into multiple cellular processes. Unlike Schwann cells which generate one myelin fragment, each oligodendrocyte can produce up to 40 myelin segments. Moreover, every axon is myelinated by several fragments (Baumann and Pham-Dinh, 2001). Electron micrographs of cross sections from the optic nerve reveal two main domains in myelin: the tightly opposed outer leaflets of the cell membrane form a less dark intraperiod line (IPL), and the closely condensed cytoplasmic membranes create the dark major dense line (MDL) (Quarles et al., 2006; Aggarwal et al., 2011a). 


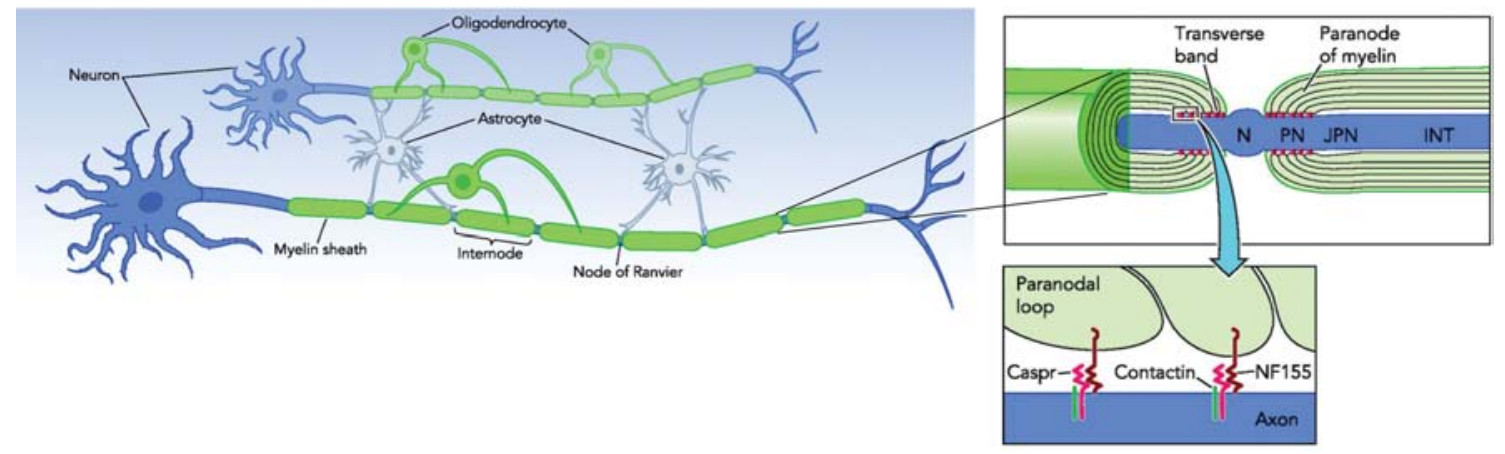

Figure 1.1: The structure of CNS myelin. In a myelinated axon four distinct regions are present; internode (INT), juxtaparanode (JPN), paranode (PN) and nodes of Ranvier (N). The figure is adapted and modified from Jackman et al. (2009). Reprint by permission from The American Physiological Society Ltd: physiology, copyright (2009).

In myelinated axons, however, four distinct domains appear: internode, juxtaparanode, paranodal loop, and nodes of Ranvier (Figure 1.1) (Jackman et al., 2009). The internode is a compacted area of myelin where different membrane layers closely interact with each other. This domain contains several characteristic proteins such as the proteolipid protein (PLP), myelin basic protein (MBP), myelin-associated oligodendrocytic basic protein (MOBP) and myelin and lymphocyte protein (MAL) (Maier et al., 2008). The most inner and outer myelin layers, however, are not compacted. These tongues encapsulate considerable amounts of cytoplasm compared to the compacted layers. The myelin internods are separated by non-myelinated nodal region where sodium channels are highly-dense clustered (Quarles et al., 2006; Baumann and Pham-Dinh, 2001). In the paranodal region the interaction between myelin and axonal surface is organized. Therefore, this domain contains specific adhesion molecules like myelin associated glycoprotein (MAG), contactin, neurofascin 155 (NF-155), neurofascin 186 (NF 186), contactin-associated protein (Caspr), and neural cell adhesion molecule (NCAM)-120 to establish the axon-glia interaction (Sherman and Brophy, 2005). The paranode is separated from the internode by the juxtaparanode domain in which potassium channels are organized (Jackman et al., 2009). Paranodes and juxtaparanodes are regions with a non-compacted myelin membrane, which are separated from the compacted area by proteins such as oligodendrocytespecific protein (OSP)/claudin 11 (Krämer et al., 2001; Maier et al., 2008). 


\subsubsection{Myelin composition}

Compared to other biological membranes, myelin unusually contains high amounts of lipids. The CNS myelin consists of $70-85 \%$ lipids and only $15-30 \%$ proteins in its dehydrated form (Quarles et al., 2006).

\subsubsection{Myelin proteins}

The major proteins in the CNS myelin are PLP, MBP, CNPase (2',3'-cyclic nucleotide 3'phosphodiesterase) and relatively low proportion of various glycoproteins.

PLP, also known as Folch-Lees protein, is a $30 \mathrm{kDa}$ transmembrane protein with a highly conserved sequence (Quarles et al., 2006). It contains four integral helix-spans into myelin membrane, interconnected by three hydrophilic loops and both $\mathrm{N}$ - and C-terminal domains of the protein are oriented towards the cytoplasm. The PLP gene is located on the X-chromosome (Williams and Deber, 1993; Möbius et al., 2008). The less abundant splice isofrom of PLP, lacking a portion of the second loop, is called DM-20 (Quarles et al., 2006). Myelin PLP and DM-20 are highly hydrophobic and by the addition of acyl chains their lipophilicity is increased during their biosynthesis and transport through the cells. This hydrophobic nature results in unusual protein properties which increase their solubility in organic solvents such as a chloroform-methanol mixture (Weimbs and Stoffel, 1992).

Together with PLP, MBP forms the major protein in the CNS myelin (Figure 1.2). Splicing of the MBP mRNA results in the production of different isoforms among which the $18.5 \mathrm{kDa}$ protein is the most abundant in human. However, in rodents $18.5 \mathrm{kDa}$ and 14 $\mathrm{kDa}$ are prominent with an increase in the ratio for $14 \mathrm{kDa}$ isoform during development. In solution, MBP has no tertiary structure, but starts to fold upon the interaction with negatively charged membranes (Quarles et al., 2006). This protein also contains phosphorylation and methylation sites which might regulate its activity (Williams and Deber, 1993). Mice lacking MBP show severe abnormality in the myelin structure especially at the end of the myelination period. The phenotype comprises severe shivering of the animal and, therefore, is called shiverer (Chernoff, 1981). 
CNPase is mainly found in the myelin inner and outer loops and in oligodendrocyte processes. It has two isoforms (46 and $48 \mathrm{kDa}$ ) in the CNS myelin that are associated with both microtubules and microfilaments (Bauer et al., 2009). In CNPase-deficient mice myelination appears normal, however, axonal swelling and neurodegeneration occurs in the adults (Lappe-Siefke et al., 2003).

CNS myelin includes several glycoproteins among which MAG and MOG (myelin oligodendrocyte glycoprotein) are most well-documented. MAG is a $100 \mathrm{kDa}$ glycoprotein and is present in the CNS and PNS. It is a single transmembrane protein with a highly Nglycosylated extracellular domain. This protein is restricted to the periaxonal surface and plays a role in the adhesion and signal transduction between glia and axonal membrane. Although, MAG knockout mice produce myelin normally, they develop periaxonal and paranodal structural deficiencies (Li et al., 1994). MOG, another myelin glycoprotein, contains a single transmembrane domain with one N-glycosylation site on the extracellular domain. This protein appears on the most outer layer of the myelin structure and is considered as a major target antigen in multiple sclerosis (Bernard et al., 1997; Quarles et al., 2006). Another myelin glycoprotein is OMgp (oligodendrocyte myelin glycoprotein) which is a phosphatidylinositol-linked membrane protein and has a molecular weight of $120 \mathrm{kDa}$. This protein plays a role in cell-cell interaction and inhibits axonal regeneration (Baumann and Pham-Dinh, 2001; Quarles et al., 2006).

Other myelin proteins are mainly the members of four membrane-spanning proteins such as MAL, plasmolipin, OSP/claudin 11, CD9 and connexins. MAL and plasmolipin are associated with glycosphingolipid-enriched membrane domains (Magyar et al., 1997; Frank, 2000; Erne et al., 2002), whereas OSP/claudin 11 is found in paranodal region and is involved in myelin radial component formation. In addition, CD9 is important in establishment of paranodal regions since the mice deficient for this protein develop myelin with abnormal paranodal loops (Ishibashi et al., 2004).

\subsubsection{Myelin lipids}

Myelin includes various types of lipids such as phospholipids, cholesterol and glycosphingolipids (GSLs) among which the last two are the main types and together compose $58 \%$ of the total myelin lipids (Jackman et al., 2009). The distribution of lipids in the myelin bi- 


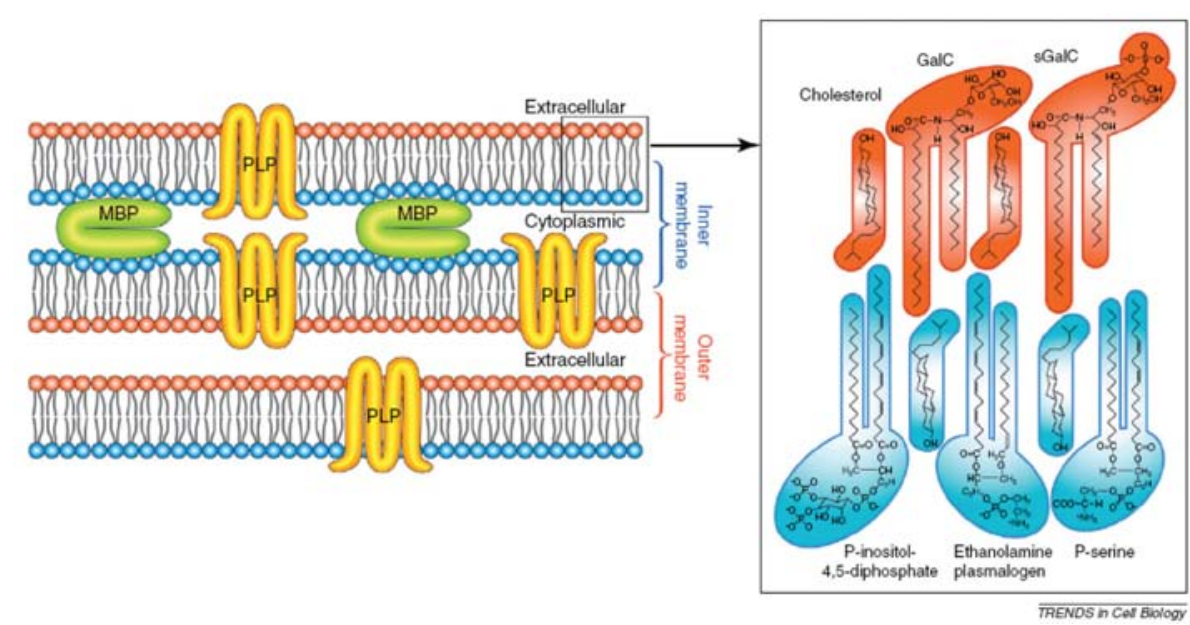

Figure 1.2: The organization of myelin compact area. PLP and MBP are the main proteins in myelin compact area. The lipids are distributed to this region asymmetrically. The figure is adapted from Aggarwal et al. (2011a). Reprint by permission from Elsevier Ltd: Trends in Cell Biology, copyright (2011).

layer is asymmetric (Figure 1.2). Whereas, ethanolamine plasmalogen, phosphatidylserine and phosphatidylinositol 4,5-bisphosphate are mainly located in the cytoplasmic leaflet, cholesterol, galactocerebrosides and phosphatidylcholine are enriched in the extracellular side (Quarles et al., 2006; Aggarwal et al., 2011a).

The main glycolipids in myelin are galactocerebrosides which include galactosylceramide (GalC) and its sulfate-derivative sulfatide. GalC is produced by addition of UDP-galactose to ceramide which is enzymatically catalyzed by UDP-galactose:ceramide galactosyltransferase (CGT). Addition of sulfate to GalC by the cerebroside sulfoteransferase (CST) enzyme produces sulfatide (Jackman et al., 2009). Studies based on antibody reactivity as well as mouse genetics have provided evidence for the role of these lipids in the formation and maintenance of myelin (Dupree et al., 1998). In cgt-null mice the velocity of nerve conduction is severely reduced to the level of unmyelinated axons. These mice develop body tremor and abnormal locomotor activity which finally leads to death at the end of myelination time. Moreover, disruption in paranodal junction and age-dependent neurological abnormalities have been reported in this mutant (Bosio et al., 1996). Interestingly, cst-null mice exhibit similar phenotype as cgt-null. Both mutants contain low levels of axonal Caspr, contactin and paranodal NF-155 in glia (Schafer et al., 2004). This indi- 
cates a role for GalC and sulfatide in the stabilization of axon-glia interaction in paranodal regions and their involvement in supporting the stability and long-term integrity of myelin membrane (Jackman et al., 2009; Aggarwal et al., 2011a).

Gangliosides are present as a minor type of lipids in the adult myelin. They are produced by transforming ceramide to glucocerbroside using the enzyme, glucosyltransferase (GlcT). This product is then converted to lactosylceramide (lacCer) by the galactosyltransferase (galT1). Different gangliosides are generated upon addition of sialic acid to lacCer by sialyltransferases. The production of GM4 is, however, different and it is directly derived from GalC by a sialyltrasnferase (Jackman et al., 2009). This lipid is the major ganglioside in the myelin of certain species, though GM1 is the main type in most vertebrates (Quarles et al., 2006). Mammalian myelin contains 0.1-0.3\% gangliosides. These lipids are important in paranodal junction stability (Maier et al., 2008). The interaction between axonal gangliosides and MAG also supports the axon-glia interaction in the paranodal area (Jackman et al., 2009). Additionally, these lipids are considered as the main myelin lipid antigens under pathological conditions such as Guillain-Barré-Syndrom (Willison, 2005).

\subsubsection{Oligodendrocyte development}

Oligodendrocytes are the responsible cells for myelin production in the CNS. The developmental steps of oligodendroglial cell lineage are tightly controlled by extrinsic and intrinsic factors. During development, oligodendrocyte progenitors are derived from ventricular and subventricular zones in the brain, as well as the ventral midline and dorsal origin in the spinal cord (Miller, 1999; Thomas et al., 2000; Bradl and Lassmann, 2010). These progenitors express PDGFR $\alpha$ (platelet derived growth factor receptor), A2B5-reactive gangliosides and NG2 surface molecules as well as many specific transcription factors (TFs) such as Nkx6.1, Nkx6.2 and Olig2. Among these, Olig2 has been found to be required for the lineage specificity (Jakovcevski et al., 2009; Emery, 2010b,a).

After cell specification, a number of transcription factors (TFs) such as Hes5, Sox5, Sox6, Id2 and Id4 keep the precursor cells (OPCs) in the proliferation stage and prevent them from entering the differentiation phase (Ndubaku and De Bellard, 2008; Emery, 2010a; Miron et al., 2011). Oligodendrocyte expansion is also promoted by growth factors and chemokines like FGF2 (fibroblast growth factor), PDGF-A, neuregulin 1, bFGF, 
neurotrophin-3 (NT3) and CXCL1 (chemokine (C-X-C motif) ligand 1) (Barres et al., 1992; Miller, 2002). Among these cues, the proliferative effect of PDGF on OPCs is well documented. PDGF is secreted by neurons and astrocytes and activates p27kip1, a molecule which enhances proliferation by inhibiting the cell-cycle regulator cyclin-dependent kinase 2 (cdk2) (Casaccia-Bonnefil et al., 1997).

Independent of their place of origin, OPCs have to travel through the brain and spinal cord to reach their final place of destination (De Castro and Bribián, 2005). This process is regulated by molecules from the surrounding environment. Some of the identified molecules responsible for contact-mediated migration of OLs are tenascin-C, PSA-NCAM, and ephrins. The OLs motility is also controlled by secreted factors such as FGF-2, PDGF, EGF (epidermal growth factor), HGF (hepatocyte growth factor), netrins and CXCL1 (Barres et al., 1992; Miller, 2002; De Castro and Bribián, 2005). In general, OPCs migration regulatory molecules include chemoattractant (PDGF and semaphorin 3F), chemorepellants (netrins, semaphorin $3 \mathrm{~A}$ and ephrins) and cues to stop motility (CLCX-1 and tenascin-C) (McTigue and Tripathi, 2008; Miron et al., 2011).

The final population of OLs is determined by a balance between their proliferation and the programmed cell death. Two major signals for oligodendrocyte survival are PDGF and insulin-like growth factors (IGFs) (Barres et al., 1992; Miller, 2002). From these, IGF-1 increases OL survival through the Akt signalling pathway (Flores et al., 2008). Furthermore, Heregulin, belonging to the neuregulin family, activates Akt through erbB receptors. Akt signalling then induces the phosphorylation of the pro-apoptotic molecule BAD (Bcl-2-associated death promoter) and consequently enhances OL survival (Flores et al., 2000). Indeed, the main sources of oligodendrocyte survival are neuronal signals, which modulate the proper number of OLs required for myelination (Bozzali and Wrabetz, 2004).

Oligodendrocyte differentiation is a complex procedure that is mainly regulated by intrinsic factors. This process depends on the inactivation of many TFs which have inhibitory effects on the OL terminal differentiation (Emery, 2010a). On the contrary, the activity of other TFs such as Sox9, Sox10, Nkx2.2 and Olig1 is necessary for OPC differentiation (Ndubaku and De Bellard, 2008; Miron et al., 2011). Also, the expression of cell surface receptors such as GPR17, which inhibit myelination process, need to be down-regulated 


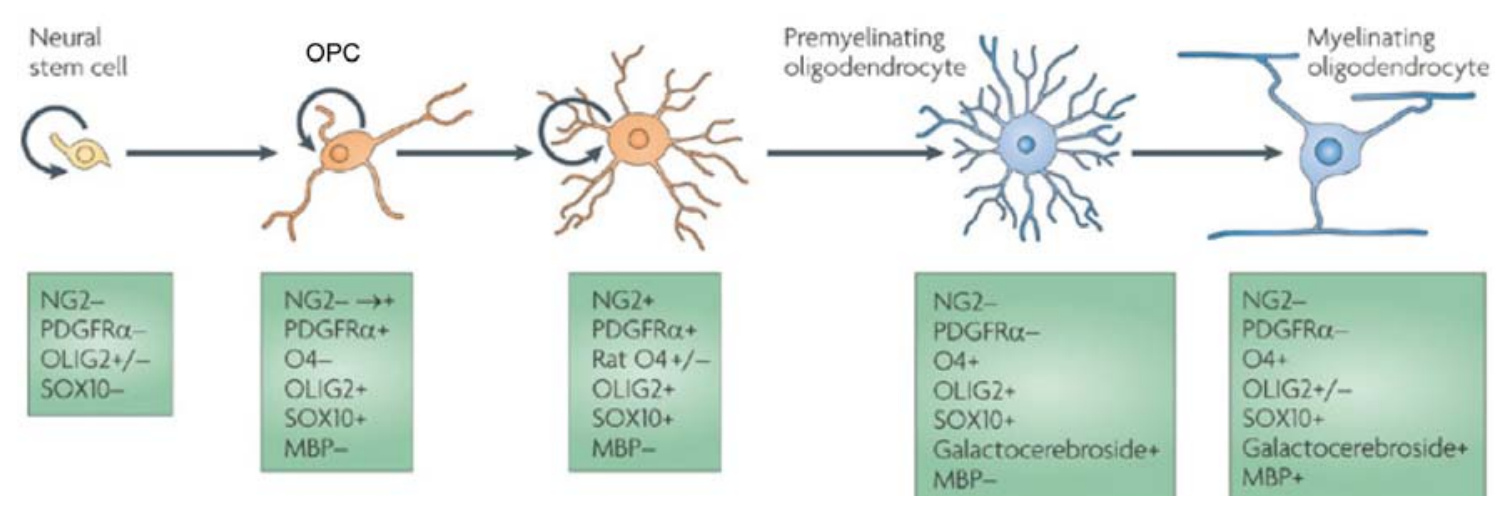

Figure 1.3: Oligodendrocyte development. Oligodendrocytes are derived from precursor cells (OPC) which are specified from neural stem cells. Proliferating OPCs then undergo differentiation to produce premyelinating oligodendrocytes. Finally, these cells become myelinating glia in response to microenvironmental signals. In each stage cells express different markers. The figure is adapted and modified from Nishiyama et al. (2009). Reprint by permission from Nature Publishing Group Ltd: Nature Reviews Neuroscience, copyright (2009).

(Chen et al., 2009).

As OLs enter terminal differentiation, they produce large amounts of myelin membrane. This stage is mainly regulated by extrinsic factors such as Lingo-1 (Leucine-rich repeat and immunoglobulin-like domain-containing nogo receptor-interacting protein-1) and PSANCAM (Rosenberg et al., 2007). Interestingly, myelin itself also inhibits the maturation of OLs (Miller, 1999). This suggests that the communication between OLs prior to myelination might be a mechanism to coordinate their process branching (Rosenberg et al., 2007) (Figure 1.3).

MicroRNAs (miRNAs) are small non-coding RNA molecules which post-transcriptionally suppress the expression of their target RNAs. These small RNAs are involved in the regulation of oligodendrocyte proliferation and differentiation as well as the myelin maintenance (Dugas and Notterpek, 2011). The main reported miRNA involved in the proliferation stage is miR-17-92 cluster. The miR-19b member of this cluster activates Akt phosphorylation through inhibition of PTEN activity and enhances cell proliferation in vitro (Budde et al., 2010). Several miRNAs, including miR-219, miR-338, miR-23a, miR-23b and miR-138, are involved in the regulation of OL differentiation (Dugas and Notterpek, 2011). From these, miR-219 suppresses PDGFR $\alpha$ and the transcription factors Sox6, Hes5, 
ZEP238 and FOXJ3 (Dugas et al., 2010). miR-338 is responsible for the silencing of the TFs Sox6, Hes5, ZEP238 and also the FGFR2 (Zhao et al., 2010). Interestingly, miR-138 promotes the expression of the genes involved in the early stage of differentiation and suppresses the expression of the late differentiation phase genes (Dugas and Notterpek, 2011).

\subsubsection{Myelin biogenesis and assembly}

In order to generate myelin segments, oligodendrocytes synthesize high amounts of lipids and proteins. The production and assembly of these components is a complex process and is initiated as oligodendrocytes enter terminal differentiation, where they express myelin genes extensively. Since myelin is a polarized structure, different biosynthetic mechanisms are involved in the regulation of its biogenesis and assembly.

One of the proposed mechanisms for the delivery of myelin components to their final destination is membrane trafficking through the biosynthetic pathway. For instance, the involvement of vesicle transport such as direct transport, trancytosis and regulated exocytosis in PLP delivery to growing membrane has been described (Krämer et al., 2001; Maier et al., 2008). Neuronal signals have been found to induce the incorporation of PLP into myelin by an increase of cAMP levels and protein kinase A (PKA) activity (Anitei and Pfeiffer, 2006; Trajkovic et al., 2006).

Although the majority of myelin components are delivered via vesicle trafficking, the incorporation of MBP and MOBP into myelin occurs differently. These proteins reach myelin by transportation of their mRNA as granules to the myelin processes where they can be locally translated (Simons and Trotter, 2007). The formation of these granules is regulated by hnRNPs and they are transported via microtubules (Maier et al., 2008; White et al., 2008; Bauer et al., 2009; White et al., 2011).

\subsubsection{Axon-glia communication}

The reciprocal communication between oligodendrocytes and neurons is critical for the coordination of myelination in the CNS (Simons and Trajkovic, 2006). It has been reported that glial cells are recruited to differentiate into myelinating cells upon receiving neuronal signals which are quantitatively related to the axonal size (Nave and Trapp, 2008). Neurons influence the developmental stages of oligodendrocytes to develop into myelinating cells. In turn, oligodendrocytes produce myelin to enable the saltatory conduction of 
action potentials and support long-term integrity of neurons (Simons and Trajkovic, 2006).

The regulatory function of neuronal extrinsic cues during myelination is important from two perspectives. They regulate the precise timing of oligodendrocyte differentiation to ensure the myelination of proper axons at the right time (Barres and Raff, 1999). For instance, neurons release Notch ligands to inhibit OL differentiation and myelination before the proper time for myelination (Barres and Barde, 2000). In addition, they control the final population of the myelinating oligodendrocytes by producing limited survival signals such as neuregulin (Fernandez et al., 2000). Many neuronal-derived growth and trophic factors such as PDGF-A, IGF-1, NT-3, FGF2 and CNTF (ciliary neurotrophic factor) have been reported to influence oligodendrocytes development. Neuregulins are neuron-derived proteins that activate the erbB family receptors in oligodendrocytes. In erbB2-deficient mice a failure in oligodendrocyte terminal differentiation and thereby axonal ensheathment has been shown (Park et al., 2001). Neuronal electrical activity also promotes myelination in the CNS by inducing the neuronal secretion of promyelinating cues like adenosine (Ishibashi et al., 2006; Demerens et al., 1996). This electrical activity also alters the expression pattern of axonal surface proteins such as PSA-NCAM (Coman et al., 2005) which is down-regulated prior to myelination (Charles et al., 2000). Neuronal signals may also regulate myelin biogenesis and assembly, by influencing vesicle trafficking in oligodendrocytes (Anitei and Pfeiffer, 2006; Trajkovic et al., 2006).

On the contrary, myelin regulates the axonal diameter by biochemical alteration of the neuronal cytoskeleton, mainly by accumulation and phosphorylation of neurofilaments (Quarles et al., 2006; McTigue and Tripathi, 2008). OLs also release trophic factors to sustain axonal survival, mediated by PI3K-Akt signalling cascade (Wilkins et al., 2003; Sherman and Brophy, 2005; Franklin and Kotter, 2008). This is supported by the finding that oligodendrocyte-derived IGF1 and GDNF (glial cell line-derived neurotrophic factor) promote axonal survival and outgrowth in cell culture, respectively (Wilkins et al., 2003; Franklin and Ffrench-Constant, 2008).

\subsubsection{Myelin pathology}

Myelin pathology can be induced by gene mutations or inflammatory immune responses targeting myelin and oligodendrocytes (Franklin and Ffrench-Constant, 2008). For ex- 
ample, Pelizaeus-Merzbacher disease (PMD) and X-linked spastic paraplegia (SPG2) are abnormalities related to mutations in the $p l p$ gene (Griffiths et al., 1998a; Readhead et al., 1994). On the other hand, multiple sclerosis (MS) and Guillain-Barré-Syndrom are diseases that arise from inflammatory immune responses targeting myelin in the CNS and PNS, respectively (Quarles et al., 1990; Sospedra and Martin, 2005).

MS is an inflammatory disease of the CNS that can result in the deficiency of sensation and motor function and lead to neurodegeneration (Sospedra and Martin, 2005). Upon inflammation in the CNS, B cells, T cells and macrophages cross the blood brain barrier (BBB) and enter the CNS, where they target OLs and myelin (Sospedra and Martin, 2005; Jessen, 2004). This leads to the production of a high amount of auto-antibodies that trigger the immune response against myelin structure and result in demyelination (Sospedra and Martin, 2005).

In MS, demyelination events are followed by remyelination processes (Franklin and FfrenchConstant, 2008). Upon injury, activated microglia and astrocytes produce growth factors and chemokines that recruit OPCs to the lesions (Franklin and Kotter, 2008; McTigue and Tripathi, 2008; Fancy et al., 2011) and induce a switch from a quiescent to an activated status in precursor cells (Franklin and Kotter, 2008). These OPCs then differentiate to myelinating glia and produce myelin, which is thinner in comparison to the myelin formed during development. However, with time remyelination declines in MS that primarily results from inefficient recruitment and differentiation of oligodendrocytes. The contribution of differentiation impairment to remyelination failure seems to increase with aging (Sospedra and Martin, 2005; Franklin and Ffrench-Constant, 2008).

\subsection{Exosomes}

Most cells release microvesicles into their surrounding space. These vesicles include shedding vesicles, apoptotic bodies and exosomes. Shedding vesicles (SVs) or ectosomes have diameter between 100 to $1000 \mathrm{~nm}$ and bleb from the plasma membrane. Their generation requires the activity of enzymes such as calpain, flippase, floppase and scrablase. Ectosomes contain selectins, integrins and metalloproteinase (Mathivanan et al., 2010) and include serine in their outer leaflet (Mittelbrunn and Sánchez-Madrid, 2012). Apop- 
totic bodies are heterogeneous vesicles with diameter from 50 to $500 \mathrm{~nm}$ and are released during programmed cell death (Mathivanan et al., 2010). Exosomes are vesicles with diameter between 40 to $100 \mathrm{~nm}$ and are released from many cells mainly hematopoietic, neural, epithelial, mast and tumour cells (Piper and Katzmann, 2007; Valadi et al., 2007). The secretion of these vesicles can be either constitutive or regulated (Lakkaraju and Rodriguez-Boulan, 2008) and they contain proteins as well as RNAs such as mRNA and microRNA (Valadi et al., 2007). Two proposed function for exosomes are disposal of surplus materials and transfer signals to the target cells (Frühbeis et al., 2012). The association of exosomes with many physiological and pathological conditions highlights the importance of these vesicles in health and disease.

\subsubsection{Composition of the exosomes}

Independent of their origin, all exosomes contain a specific subset of proteins such as alix, TSG101 (tumor susceptibility gene 101), Rab GTPases, SNAREs (Soluble NSF Attachment Protein Receptors) and annexins which are involved in their biogenesis, transport and fusion (Simons and Raposo, 2009). Further components of exosomes are tetraspanins (CD63, CD9, CD81 and CD82), adhesion molecules (MFG-E8 and integrins), heat shock (Hsc70 and Hsc90), cytoskeletal proteins (actin, cofilin, tubulin and moesin) and raft associated proteins (flotillin, stomatin, lyn and CD55) (De Gassart et al., 2004; Février and Raposo, 2004; Simons and Raposo, 2009). However, depending on their origins, exosomes may contain some specific proteins. For instance, hematopoietic cells-derived exosomes carry antigen presenting proteins such as CD86, MHC I and II (Février and Raposo, 2004). Exosomes are furthermore enriched in raft-lipids, such as cholesterol, sphingolipids and ceramide (Figure 1.4 A) (Simons and Raposo, 2009).

\subsubsection{Formation and release of exosomes}

Exosomes are generated from multivesicular bodies (MVBs) by inward budding of the membranes (De Gassart et al., 2004). MVBs are a part of the endosomal system which includes primary endocytic vesicles, early and late endosomes (EEs and LEs) as well as lysosomes (Denzer et al., 2000). The invagination of the limiting membrane of endosomes results in the formation of intraluminal vesicles (ILVs) of MVBs (Hurley and Hanson, 2010). These MVBs will face two different fates; fusion with the lysosmes to degrade 

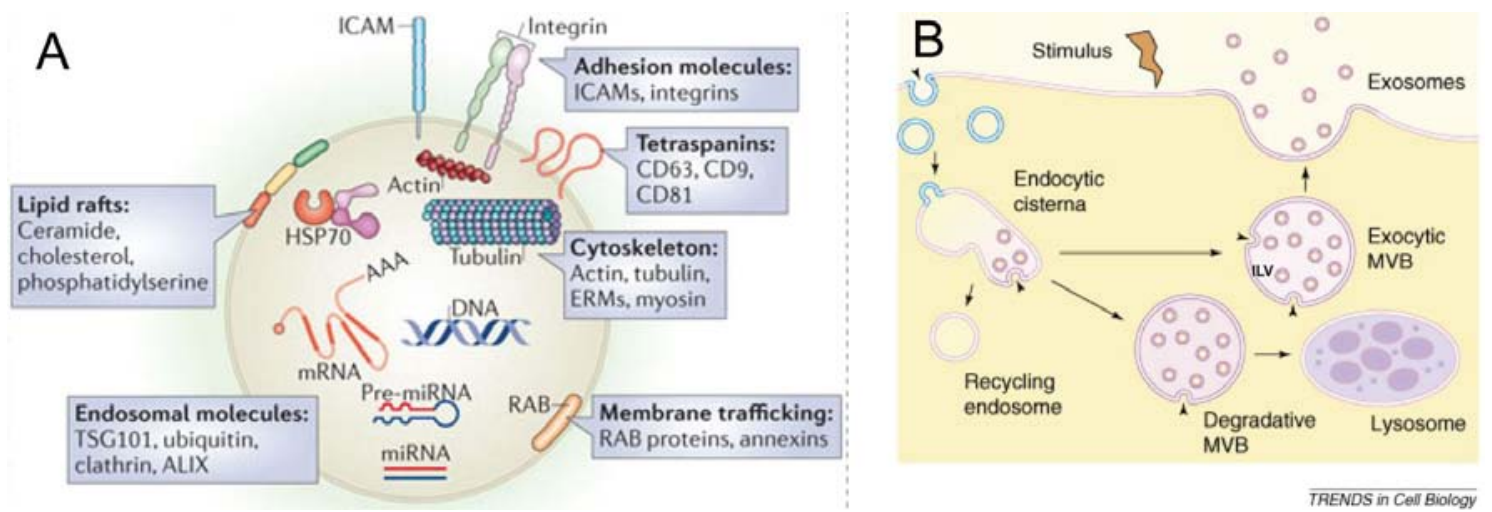

Figure 1.4: Exosome composition and formation. (A) Exosomes contain many proteins involved in their biogenesis and release. They also include specific lipids and carry nucleic acids. The figure is adapted and modified from Mittelbrunn and Sánchez-Madrid (2012). Reprint by permission from Nature Publishing Group Itd: Nature reviews molecular cell biology, copyright (2012). (B) Exosomes are generated from inward budding of multivesicular bodies (MVBs). MVBs are a part of the endosomal system and are generated by the invagination of late endosomes to produce intraluminal vesicles (ILVs). The figure is adapted and modified from Cocucci et al. (2009). Reprint by permission from Elsevier Ltd: Trends in Cell Biology, copyright (2009).

their contents, and fusion with the plasma membrane to release their components into extracellular space (Figure 1.4 B) (Denzer et al., 2000; Keller et al., 2006). Once generated, the destination of MVBs depends on their molecular composition. For instance, ILVs enriched in ubiquitinated proteins and phosphatidylinositol-3-phosphate are delivered to lysosomes, whereas vesicles rich in lysobisphosphatidic acid (LBPA) may undergo nondegradative pathway (Van Der Goot and Gruenberg, 2006; Subra et al., 2007).

The insertion of proteins into MVBs is mediated by two mechanisms; mono-ubiquitination of target protein and non-ubiquitin sorting mechanism, which requires signals such as tetraspanin enrichment or cholesterol enriched domains (Keller et al., 2006; Piper and Katzmann, 2007). The insertion of ubiquitinated proteins into MVBs is carried out by ESCRT (endosomal sorting complex required for transport) machinery which include four complexes and some associated proteins. First, the ESCRT protein Vsp-27 identifies the ubiquitinated cargos and then engages other ESCRT complexes as well as TSG101 to activate AIP/Alix which inserts the target into the budding vesicles (Février and Raposo, 2004; Johnstone, 2006; Keller et al., 2006). Alix is a linker between ESCRT complexes and together with TSG101 is a part of ESCRT I complex (Lakkaraju and Rodriguez- 
Boulan, 2008). While ESCRT I and II recognize ubiquitinated proteins, ESCRT III is involved in membrane budding (Simons and Raposo, 2009). Finally ESCRT complexes release the vesicle into MVBs intraluminal space using their intrinsic budding and scission function (Hurley and Hanson, 2010). After formation, MVBs are transported to lysosomes or plasma membrane by the docking and fusion that is regulated by Rabs and SNAREs (Denzer et al., 2000; Février and Raposo, 2004). Many members of Rab GTPase family are involved in exosomes formation and release. Whereas Rab4, Rab5 and Rab11 are involved in early recycling endosomes, Rab7 and Rab9 participate in the formation of late endosomes (Mathivanan et al., 2010). From these, Rab11 induces exosomes formation in K562 (Fader et al., 2005) and their release in dendritic cells (Lakkaraju and Rodriguez-Boulan, 2008). Other Rabs involved in exosome release are Rab35, Rab27a and Rab27b (Bobrie et al., 2011; Hsu et al., 2010). Moreover $\mathrm{Ca}^{2+}$ is also essential for exosome formation and release (Johnstone, 2006).

\subsubsection{Function of exosomes}

Exosomes are involved in horizontal transfer of proteins and RNAs between cells and therefore are considered as a novel type of communication (Valadi et al., 2007; Lai and Breakefield, 2012). For instance, exosomes from differentiated reticulocytes and platelets possess clearance and aggregatory function, respectively (Rabesandratana et al., 1998; Heijnen et al., 1999). Exosomes also play a role in antigen presentation and development of tolerance (Théry, 2011). They might be also involved in the establishment of cell polarity and tissue patterning (Lakkaraju and Rodriguez-Boulan, 2008). To fulfill these functions exosomes can interact with target cells via three different mechanisms; binding to the plasma membrane surface, fusion with the target membrane, or internalization by the recipient cells (Denzer et al., 2000; Valadi et al., 2007). The best examples of exosomal function have been described in antigen presentation and tumour metastasis (Lakkaraju and Rodriguez-Boulan, 2008).

Exosomes are widely used for communication between immune cells (Keller et al., 2006). They increase the immunological responses by inducing the activation of $\mathrm{T}$ cells (Johnstone, 2006). For instance, antigen presenting cells (APCs) such as B cells and dendritic cells (DCs) release exosomes containing MHC peptides for presenting to specific $\mathrm{T}$ cells to influence adaptive and anti-tumour immune responses, respectively (Théry, 2011). The 
vesicles from dendritic cells also recruit more DCs presenting MHCs (Bobrie et al., 2011).

Several human tumours such as melanoma, colon, ovarian, breast, and prostate carcinoma secrete exosomes. Tumour-derived exosomes affect the immune system by inducing apoptosis in activated anti-tumour $\mathrm{T}$ cells and impair dendritic cell maturation (Iero et al., 2008). They also inactivate $\mathrm{T}$ cells and natural killer cells and induce differentiation of regulator T cells or myeloid cells to suppress immune system (Bobrie et al., 2011; Théry, 2011). In addition, tumour-derived exosomes possess self-promoting effect and induce the proliferation of their cells of origin (Skog et al., 2008). They also contain RNAs and induce angiogenesis in endothelial cells (Mittelbrunn and Sánchez-Madrid, 2012). In melanoma patients, exosomes induce angiogenesis and metastatic invasion which produce a tumour niche by altering ECM (Somasundaram and Herlyn, 2012).

Exosomes play a role in the nervous system during development and in disease (Lai and Breakefield, 2012). MVBs from neurons contain mRNAs and are present in both pre- and post-synaptic structures (Chivet et al., 2012). The functions of exosomes in the neural system under physiological conditions include neural development (such as axon pathfinding), synaptic activity (L1 and GluR2/3 containing MVBs), regeneration, and axon-glia communication. They are also involved in pathological conditions such as neurodegenerative diseases (Lai and Breakefield, 2012).

Recent studies have suggested a contribution of exosomes in the progression of neurodegenerative diseases such as Alzheimer's (AD), Parkinsons's and ALS (amyotrophic lateral sclerosis) diseases (Vella et al., 2008). In Alzheimer's disease (AD), $\beta$-amyloid peptides $(\mathrm{A} \beta)$ are produced by proteolytic cleavage of the amyloid precursor protein (APP) (Cai et al., 1993). Minor fractions of $\mathrm{A} \beta$ have been found to be released by exosomes (Rajendran et al., 2006). Accordingly, an accumulation of the exosomal proteins such as alix and flotillin in the brain plaques from AD patients has been detected (Kokubo et al., 2000; Rajendran et al., 2006). Moreover, exosomes from prion protein-expressing cells carry both naturally $\left(\mathrm{PrP}^{\mathrm{c}}\right)$ and abnormally-folded $\left(\mathrm{PrP}^{\mathrm{sc}}\right)$ protein variants (Fevrier et al., 2004). These proteins are involved in disorders such as CJD, GSS, BSE and scrapie (Chakraborty et al., 2005). Interestingly, exosomes carrying $\mathrm{PrP}^{\mathrm{sc}}$ can induce the conversion of endogenous $\mathrm{PrP}^{\mathrm{c}}$ to $\mathrm{PrP}^{\mathrm{sc}}$ in the non-infected target cells (Vella et al., 2007). As exosomes circulate in body fluids such as serum, urine and CSF (Cerebrospinal fluid) and contain 
disease related proteins, they are considered as potential markers in pathological conditions (Bellingham et al., 2012).

\subsection{Cell adhesion and glycocalyx}

\subsubsection{Cell adhesion}

Cell adhesion influences basic cell functions such as proliferation, survival, motility and differentiation (Vitte et al., 2005). The adhesion between two biological membranes is regulated by different forces including specific interaction between cell surface receptors, non-specific or generic forces, and membrane elasticity. This dynamic process involves a series of steps to modulate the contact between two opposite membranes. To expose the hidden ligand and receptors from the membrane surface, the repulsive molecules, mainly glycocalyx, should be excluded. This leads to homophilic or heterophilic interaction between cell adhesion molecules and thereby formation of a tight interacting zone, a process involving receptor segregation. Moreover, signalling associated with adhesion event modulates the long term interaction between two membranes. (Sackmann and Bruinsma, 2002). The main specific receptors involved in adhesion process are classified into integrins, immunoglobulin superfamily/IgSF (NCAM, CD2 and CD58), selectins (CD62-P, CD62-E), mucine-like molecules (leukosialin/CD43), and cadherins (Vitte et al., 2005).

\subsubsection{Glycocalyx}

Glycocalyx is a carbohydrate-rich layer associated with the cell membrane surface. It comprises of a wide variety of membrane associated macromolecules including glycoproteins, glycolipids, and proteoglycan (Weinbaum et al., 2007; Curry and Adamson, 2011). Glycoproteins contain relatively short oligosaccharide chains (2-15 residues), especially sialic acid with branched carbohydrate sequences. Major components of this group are cell adhesion molecules and coagulation and fibrinolysis elements. Proteoglycans are proteins with a covalently associated sulfated glycosaminoglycans (GAGs) side chain which provide a net negative charge due to the polyanionic nature of its components. GAGs are classified into five groups; heparin sulfate (HS), keratin sulfate, dermatan sulfate, chondroitin sulfate (CS), and hyaluronan or hyaluronic acid (Reitsma et al., 2007; Weinbaum et al., 2007). The glycocalyx also involves soluble components that are connected to the plasma 
membrane via proteoglycans and glycoproteins (Reitsma et al., 2007). Other components of this structure are enzymes, enzyme inhibitors, growth factors, cytokines, cationic amino acids, and cations (Weinbaum et al., 2007). The composition and interaction of glycocalyx elements is highly dynamic. Therefore, removal of an individual constituent of the layer influences its properties, highlighting the importance of the interactions within the intact architecture (Reitsma et al., 2007). Cells can modulate the glycocalyx to adjust their adhesion under physiological state (Vitte et al., 2005). Many observations also indicate a role for the glycocalyx in mechano-transduction. This occurs by transmitting fluid shear stress into cells through glycocalyx components which in turn induces rearrangement of actin cytoskeleton and generates signalling events (Tarbell and Pahakis, 2006).

\subsubsection{Glycocalyx involvment in cell adhesion}

Glycocalyx contains a huge density of repeller molecules, which modulate the contact between two adhering cells (Sackmann and Bruinsma, 2002). Besides the participation of glycan structures in interaction between cells as a simple bridge, they can also regulate adhesion by non-specific activity (Taylor and Drickamer, 2007). Living cells control their adhesive capacity by adjusting the amount of glycocalyx, which generates non-specific repulsion and impairs adhesion more efficiently under dynamic conditions (Sabri et al., 1995, 2000). In the presence of glycocalyx, despite the expression of adhesion molecules the interaction between two membranes may not occur due to negative regulation by nonspecific repulsive forces (Foa et al., 1996). Thus, relative length of adhesion elements together with surface repeller density, are the key parameters for determination of steric (physical) stabilization between different cells. For instance, elimination of a glycocalyx component, CD43, from THP-1 cells membrane surface, increases their adhesiveness under physiological condition, which might be an expedient way for cells to induce rapidly the activity of many existed receptors on their membranes (Foa et al., 1996; Sabri et al., 2000). Furthermore, glycocalyx attenuates the interaction between blood cells and vessel wall. It also prevents the interaction of platelets to endothelium under physiological condition, whereas partial removal of glycocalyx increases platelet adhesion to vessel wall (Reitsma et al., 2007).

The pericellular matrix is composed of a variety of carbohydrates that include anionic groups such as sialic acid and sulfate group which consequently give the living cells a net negative charge surface. This electrically charged cell-coat produce a dynamic repulsive 
barrier preventing cell-cell interaction efficiently (Sabri et al., 1995). The steric repulsion relies on the ionic strength. The restoring of the inhibitory effect on the cell interaction by monovalent salt indicates the involvement of negative charge in the contact process (Johnson et al., 2005). For example, endothelial glycocalyx permeability is dependent on size, steric hindrance, and electrostatic charge. An increase in albumin uptake and labeled dextran internalization has been reported upon neutralization of the glycocalyx, underscoring the involvement of electrostatic charges in the process (Reitsma et al., 2007).

\subsubsection{Sialic acid and its polymer PSA}

It has been proposed that sialic acid is the main molecule to prevent the intermembrane contact by steric inhibition (Fujimoto et al., 2001). Consistent with this hypothesis the electrostatic repulsion from this molecule, incorporated into glycoproteins, induces the separation of endothelial cell surface and thereby lumen formation. This mechanism may apply to initiate lumen development in other organs (Strilić et al., 2010). However, the main form of sialic acid, which is involved in preventing membrane adhesion, is polysialic acid (PSA). PSA is a long, negatively charged, linear carbohydrate polymer which is produced by $\alpha 2,8$-linked interaction between $\mathrm{N}$-acetylneuraminic acid (Neu5Ac) residues. This linear polymer is post-translationally attached to proteins such as NCAM (Johnson et al., 2005). The regulatory function of PSA on membrane adhesion is dependent on its abundance, massive volume in hydrated form, and slippery nature. This sialic acid polymer has been shown to have a direct effect on distance between membranes by generating a physical repulsion rather than a receptor binding activity (Rutishauser, 2008). Moreover, PSA influences the adhesion properties of other proteins in the membrane surface. This effect is a general characteristic of PSA rather than the interfering with a specific interaction (Taylor and Drickamer, 2007). The biological function of adding PSA to NCAM has been revealed to influence global cell interaction rather than NCAM activity (Fujimoto et al., 2001). On the other hand, the main function of polysialylation is to prevent NCAM-mediated adhesion that consequently inhibits the interaction between other protein receptors due to large volume of hydrated PSA (Taylor and Drickamer, 2007; Rutishauser et al., 1990). For instance, the cadherin-dependent adhesion has been reported to be attenuated upon increase in the ratio of PSA-NCAM to this protein (Johnson et al., 2005). 


\subsection{Aims of the study}

Exosomes are microvesicles which are considered as a novel system for cell communication. Oligodendrocytes, as the myelinating cells in the central nervous system, are known to produce exosomes. The function of these vesicles in oligodendrocytes, however, has not been elucidated yet. In the first part, we investigated the role of oligodendrocytederived exosomes during axon-glia interaction in a cell culture model system. We also studied the molecular mechanisms used by these vesicles to exert their effect. Moreover, we explored the possible regulatory effect of neuronal signals on exosomes release by oligodendrocytes. The answers to these questions could provide evidence for the physiological role of oligodendrocyte-derived exosomes in the CNS.

In the second part, we investigated the mechanisms involved in the adhesion and compaction in the extracellular leaflets of the myelin membrane. We explored the putative role of proteolipid protein (PLP) in the adhesion of myelin extracellular side. We also analyzed glycocalyx alteration during oligodendrocyte maturation and investigated whether the removal of this carbohydrate-rich layer influences myelin adhesiveness. Addressing these questions might provides more insight into the assembly and stability of myelin membrane structure in the central nervous system. 


\section{Chapter 2}

\section{Materials and Methods}

\subsection{Materials}

\subsubsection{Chemicals and consumables}

All the chemicals were purchased from Sigma-Aldrich GmbH (Munich, Germany), AppliChem GmbH (Darmstadt, Germany) or Merck KGaA (Darmstadt, Germany) unless stated. All media, supplements, antibiotics and sera for cell culture were obtained from PAA Laboratories GmbH (Pasching, Austria) or Gibco/Invitrogen GmbH (Darmstadt, Germany). The consumable materials for cell culture, biochemical analysis, and molecular biology were supplied by Eppendorf AG (Hamburg, Germany), Falcon (Becton Dickinson Labware Europe, Le Pont De Claix, France) and Greiner Bio-One (Greiner Bio-One GmbH, Frickenhausen, Germany). The tubes for ultracentrifugation were purchased from Beckman (Beckman Coulter GmbH, Krefeld, Germany).

The chemical components and inhibitors listed in table 2.1 were purchased from SigmaAldrich, Munich, Germany.

Table 2.1: Inhibitors and chemical components

\begin{tabular}{lll}
\hline Name & Application & Solvent \\
\hline \hline Y27632 & $10 \mu \mathrm{M}$ & DMSO \\
Belbbistatin & $50 \mu \mathrm{M}$ & DMSO \\
PP2 & $1 \mu \mathrm{M}$ & DMSO \\
Dextran & $5 \mathrm{mg} / \mathrm{mL}$ & Water \\
Dextran sulfate & $5 \mathrm{mg} / \mathrm{mL}$ & Water \\
Protamine sulfate & $50 \mu \mathrm{g} / \mathrm{mL}$ & Water \\
\hline
\end{tabular}




\subsubsection{Antibodies and labeling components}

Primary antibodies which are listed in table 2.2 were used in this study. Biotinylated Maachika amurensis lectin II (MAL II) were obtained from Vector lab., Burlingame, CA, USA.

Table 2.2: Primary antibodies

\begin{tabular}{|c|c|c|c|}
\hline Target & Host species & "Application" 1 & Resource \\
\hline A2B5 & Mouse IgM & IF $(1: 200)$ & Millipore \\
\hline Actin (AC-40) & Mouse IgG2a & WB (1:500) & Sigma-Aldrich \\
\hline Akt (pan) & Rabbit & WB $(1: 1000)$ & Cell Signaling \\
\hline Akt (p-Ser-473) & Rabbit & WB $(1: 1000)$ & Cell Signaling \\
\hline Alix/AIP & Mouse IgG1 & WB $(1: 1000)$ & BD Biosciences \\
\hline Calnexin & Rabbit & WB $(1: 1000)$ & Stressgen Bioreag. \\
\hline CNPase & Mouse IgG1 & IF (1:200), WB (1:500) & Sigma-Aldrich \\
\hline FAK & Mouse IgG1 & WB $(1: 1000)$ & BD Transd. Lab. \\
\hline FAK (p-Tyr-397) & Rabbit & WB $(1: ! 000)$ & Invitrogen $\mathrm{GmbH}$ \\
\hline Flotillin-2 & Mouse IgG & WB $(1: 1000)$ & BD Biosciences \\
\hline Fyn $(H-80)$ & Rabbit & WB $(1: 200)$ & Santa Cruz Biot. \\
\hline GFAP & Mouse IgG & IF $(1: 100)$ & Santa Cruz Biot. \\
\hline Iba1 & Rabbit & IF $(1: 200)$ & Wako Chem. GmbH \\
\hline MAG (clone 513) & Mouse IgG1 & WB (1:500) & (Poltorak et al., 1987) \\
\hline MBP & Mouse IgG1 & IF $(1: 1000)$ & Sternberger \\
\hline MBP & Rabbit & IF $(1: 400)$, WB $(1: 1000)$ & DakoCytomat. \\
\hline MLC2 & Rabbit & WB $(1: 1000)$ & Cell Signaling \\
\hline MLC2 (p-Ser-19) & Rabbit & WB $(1: 1000)$ & Cell Signaling \\
\hline MOG (clone 8-18-C5) & Mouse IgG1 & IF (1:100), WB (1:500) & Millipore \\
\hline Myc & Rabbit IgG & IF $(1: 500)$ & Sigma-Aldrich \\
\hline NogoA (11C7) & Mouse IgG & WB $(1: 10000)$ & From M. Schwab \\
\hline $\mathrm{O} 1$ & Mouse IgM & IF $(1: 50)$, DB (1:100) & (Sommer and Schachner, 1981) \\
\hline $\mathrm{O} 4$ & Mouse IgM & IF $(1: 50)$ & (Kuhlmann-Krieg et al., 1988) \\
\hline PLP (clone 3F4) & Mouse IgG & WB (1:100) & (Greer et al., 1992) \\
\hline PLP (P6) & Rabbit & IF $(1: 300)$ & (Linington and Waehneldt, 1990) \\
\hline PLP (AA3) & Mouse IgG & WB $(1: 150)$ & Clone from K. Nave \\
\hline RhoA & Rabbit & WB (1:1000) & Cell Signaling \\
\hline Ribosomal S6 (p-Ser-235/236) & Rabbit & WB $(1: 1000)$ & Cell Signaling \\
\hline SFK (p-Ty r-529) & Rabbit & WB $(1: 1000)$ & Calbiochem/Merck KGaA \\
\hline SFK (p-Tyr-418) & Rabbit & WB $(1: 1000)$ & Calbiochem/Merck KGaA \\
\hline ST8SIA4 & Rabbit & WB $(1: 1000)$ & Sigma-Aldrich \\
\hline TSG-101 & Mouse IgG1 & WB $(1: 1000)$ & GeneTex Inc. \\
\hline Tubulin, $\beta$-III & Mouse IgG1 & IF $(1: 3000)$ & Promega \\
\hline
\end{tabular}

\footnotetext{
${ }^{1}$ IF: immunocytochemistry, WB: western blot, DB: dot blot
} 
The secondary antibodies and fluorophore-coupled compounds used in this study are listed in table 2.3 .

Table 2.3: Secondary antibodies and fluorophore-coupled compounds

\begin{tabular}{ll}
\hline Name & Obtained from \\
\hline \hline HRP-anti mouse IgG & Dianova, Hamburg, Germany \\
HRP-anti mouse IgM & Dianova, Hamburg, Germany \\
HRP-anti rabbit & Dianova, Hamburg, Germany \\
Alexa Fluor 488 anti rabbit & Invitrogen GmbH,Darmstadt, Germany \\
Alexa Fluor 488 anti mouse IgG & Invitrogen GmbH, Darmstadt, Germany \\
Alexa Fluor 488 anti mouse IgM & Invitrogen GmbH, Darmstadt, Germany \\
Cy3 anti mouse IgG & Dianova, Hamburg, Germany \\
Cy3 anti mouse IgM & Dianova, Hamburg, Germany \\
Cy5 anti mouse IgG & Dianova, Hamburg, Germany \\
Cy5 anti rabbit & Dianova, Hamburg, Germany \\
DyLight anti rabbit IgG & Dianova, Hamburg, Germany \\
Alexa Fluor 647 anti mouse IgG & Invitrogen GmbH, Darmstadt, Germany \\
Alexa Fluor 488 streptavidin & Invitrogen GmbH, Darmstadt, Germany \\
Alexa Fluor 647 ConA & Invitrogen GmbH, Darmstadt, Germany \\
Cell mask orange & Invitrogen GmbH, Darmstadt, Germany \\
\hline
\end{tabular}

\subsubsection{Commercial kits}

The commercial kits used in this investigation are listed in table 2.4.

Table 2.4: Commercial kits

\begin{tabular}{lll}
\hline Kit & Application & Producer \\
\hline \hline BrdU in situ detection kit & Cell proliferation & BD Biosciences \\
ConA isolation kit & Glycoprotein isolation & Thermo scientific \\
Fluorescein lectin kit I & Lectin staining & Vector lab. \\
PKH26 Red Fluorescent Cell Linker & General Cell Membrane Labeling & Sigma-Aldrich \\
PKH67 Green Fluorescent Cell Linker & General Cell Membrane Labeling & Sigma-Aldrich \\
Rhotekin RBD, agarose & RhoA-GTP pull-down & Millipore \\
Click-iT@ Cell Reaction Buffer Kit & Metabolic labeling & Invitrogen GmbH \\
\hline
\end{tabular}

\subsubsection{Animals}

The following mouse and rat strains were utilized in this study. 
Table 2.5: Mouse and rat strains

\begin{tabular}{lll}
\hline Strain & Genetic alteration & Origin \\
\hline \hline NMRI WT mice & No mutation & Animal core facility, MPI of Experimental Medicine \\
C57/N WT mice & No mutation & Animal core facility, MPI of Experimental Medicine \\
KPLP mice & PLP knockout & K.-A. Nave (Klugmann et al., 1997) \\
Shiverer mice & MBP knockout & (Chernoff, 1981) \\
PLP-PST (tg246) & ST8SiaIV overexpression & M. Eckhardt (Fewou et al., 2007) \\
WT Rat & No mutation & Animal core facility, MPI of Experimental Medicine \\
\hline
\end{tabular}

\subsubsection{Plasmids}

The following expression plasmids which have been previously published, were used: PLPmyc from Trajkovic et al., 2006, pcDNA3.1-S-MAG N ter (truncated) and pcDNA3.1-SMAG N terC36 (full length) from Aggarwal et al., 2011b.

\subsubsection{Media and sera}

\subsubsection{Commercial media, sera and solutions}

Table 2.6: Commercial media, sera and solutions

\begin{tabular}{ll}
\hline Media, sera, and solution & Manufacturer \\
\hline \hline B-27 supplement & Gibco/Invitrogen GmbH, Darmstadt, Germany \\
Basal Medium Eagle (BME) & Gibco/Invitrogen GmbH, Darmstadt, Germany \\
Dulbecco's Modified Eagle Medium (DMEM) & Gibco/Invitrogen GmbH, Darmstadt, Germany \\
Dulbecco's Modified Eagle Medium (DMEM) & PAA Laboratories GmbH, Pasching, Austria \\
Fetal Calf Serum (FCS) & PAA Laboratories GmbH, Pasching, Austria \\
GlutaMAX ${ }^{T M}$ supplement & Invitrogen GmbH, Darmstadt, Germany \\
HEPES buffer & AppliChem GmbH,Darmstadt, Germany \\
Horse Serum (HS) & PAA Laboratories GmbH, Pasching, Austria \\
Insulin-Transferrin-Selenium - A supplement 100× & Invitrogen GmbH, Darmstadt, Germany \\
LB medium and LB agar plate & AppliChem GmbH, (Darmstadt, Germany \\
L-Thyroxine & Calbiochem/Merck KGaA, Darmstadt, Germany \\
Opti-MEM + GlutaMAX ${ }^{\top M}$ & Invitrogen GmbH, Darmstadt, Germany \\
Phosphate Buffered Saline (PBS) & PAA Laboratories GmbH, Pasching, Austria \\
Penicillin/Streptomycin (Pen/Strep) 100× & Invitrogen GmbH, Darmstadt, Germany \\
Sodium Pyruvate & Gibco/Invitrogen GmbH, Darmstadt, Germany \\
Triiodothyronine & Calbiochem/Merck KGaA, Darmstadt, Germany \\
Trypsin-EDTA & Gibco/Invitrogen GmbH, Darmstadt, Germany \\
Mowiol & Calbiochem/Merck KGaA, Darmstadt, Germany \\
\hline
\end{tabular}




\subsubsection{Meida preparation}

\section{PTK2 cells medium}

$110 \mu \mathrm{g} / \mathrm{ml}$ Pyruvate

$$
\begin{aligned}
10 \% & \text { FCS } \\
1 \times & \text { Pen/Strep } \\
1 \times & \text { GlutaMAX }{ }^{\top M}
\end{aligned}
$$

in DMEM with high glucose and without glutamine from PAA.

\section{BME mixed medium}

$$
\begin{aligned}
& 10 \% \text { HS } \\
& 1 \times \text { Pen/Strep } \\
& 1 \times \text { GlutaMAX } \\
& \text { in BME medium. }
\end{aligned}
$$

\section{Super SATO medium}

$$
\begin{aligned}
2 \%(50 \times) & \text { B27-supplement } \\
1 \% & \text { Horse serum } \\
110 \mu \mathrm{g} / \mathrm{ml} & \text { Pyruvate } \\
500 \mathrm{pM} & \text { Tri-iodo-thyronine } \\
520 \mathrm{nM} & \text { L-thyroxine } \\
1 \times & \text { Pen/Strep } \\
1 \times & \text { GlutaMAX }
\end{aligned}
$$

in DMEM with high glucose and without glutamine from Gibco/Invitrogen.

\subsubsection{Softwares}

Table 2.7: Softwares

\begin{tabular}{ll}
\hline Softwares & Source \\
\hline \hline Adobe Illustrator CS 3 & Adobe Systems Incorporated,California, USA \\
GraphPad Prism & GraphPad Software, Inc., California, USA \\
ImageJ & http://rsbweb.nih.gov/ij/ \\
Leica Confocal Software, 2.61 & Leica, Mannheim, Germany \\
Meta Imaging Series 6.1 & Universal Imaging Corp., USA \\
\hline
\end{tabular}




\subsection{Methods}

\subsubsection{Cell culture and transfection}

\subsubsection{Primary culture}

Primary cultures of rat or mouse oligodendrocytes from postnatal day 1 were prepared as described previously (Trajkovic et al., 2006). Neonatal brain hemispheres were stripped free of meninges, digested and cultured in Eagle 's basal medium with $10 \%$ horse serum on poly-L-lysine-coated flask at $37{ }^{\circ} \mathrm{C}$. After 8-10 days, oligodendrocytes were harvested from mixed glia culture using mechanical dissociation. Isolated cells were then cultured in DMEM containing B-27 supplement, 1\% horse serum, triiodothyronine, L-thyroxine, pyruvate, glutamine, and penicillin/streptomycin on poly-L-lysine-coated glass coverslips or dishes.

Microgilal and astrocyte cells were isolated from the same mixed glia culture, used for OLs purification. After 10 days of culture and removing of oligodendrocytes, the remaining cells were trypsinized and plated on poly-L-lysine-coated glass coverslips in DMEM containing 10\% FCS, glutamine, and penicillin/streptomycin. After 3 days, the cells were used for further analysis.

Primary neuronal cultures were obtained by preparing a mixed brain culture from E16 (embryonic day 16) mice (Fitzner et al., 2006). The cells were plated and cultured for 2 weeks in super sato medium. The neuronal conditioned medium was obtained from these cultures, collected after culturing for 2 weeks, centrifuged for $10 \mathrm{~min}$ at 1,500 rpm, and used. Coculture of neurons and oligodendrocytes were prepared by adding of 30,00050,000 OLs to 2 week old neuronal cells cultured on coverslips.

\subsubsection{PtK2 cells}

The cells were maintained in DMEM medium with 10\% FCS in flasks with regular passaging (Eggeling et al., 2009). For experimental procedure, cells were trypsinized and then plated on glass coverslips. After obtaining desired confluency, cells were used for assays. 


\subsubsection{Transfection}

Primary oligodendrocytes transfection was performed using lipofectamine transfection reagent (Gibco/Invitrogen GmbH, Darmstadt, Germany). $1.6 \mu \mathrm{g}$ of plasmid DNA and $4 \mu \mathrm{l}$ of lipofectamine were added to $100 \mu \mathrm{l}$ Opti-MEM separately and incubated for $5 \mathrm{~min}$ at room temperature. The fractions were then mixed and incubated further for $25 \mathrm{~min}$ and then added to 4-day-old primary oligodendrocytes for $16 \mathrm{hr}$.

For PtK2 cell line, Trans-IT transfection reagent (Mirus Biologicals LLC, Madison, WI, USA) was applied to deliver mammalian expression vector into the cells. The cells were cultured till 60-75\% confluency on the day of transfection. $3 \mu \mathrm{l}$ of transfection reagent and $1 \mu \mathrm{g}$ of plasmid DNA were added subsequently to $100 \mu \mathrm{l}$ Opti-MEM and the mixture was incubated at room temperature for 25-35 min. The mixture was then introduced to the cells with gentel shaking and the cells were incubated further for 16 to $24 \mathrm{hr}$.

\subsubsection{Exosomes Preparation}

Exosomes were prepared using differential centrifugation steps (Hsu et al., 2010) which were carried out by a Beckman XL-70 Ultracentrifuge (Beckman Coulter GmbH, Krefeld, Germany). After 6 to 8 day of OLs culturing, the medium was collected, and then centrifuged for $10 \mathrm{~min}$ at $3,000 \times \mathrm{g}$ and two times for $10 \mathrm{~min}$ at 4,000 $\times \mathrm{g}$ before subjecting it for ultracentrifugation at 10,000 $\times \mathrm{g}$ for $30 \mathrm{~min}$ and at 100,000 $\times \mathrm{g}$ for $1 \mathrm{hr}$. The collected pellets were finally resuspended in PBS, medium or sample buffer.

\subsubsection{Proliferation assay}

Proliferation of OLs was assessed using BrdU in situ detection kit. Briefly, one day after shaking, OLs were incubated with $10 \mu \mathrm{M}$ Bromodeoxyuridine (BrdU) and proliferation assay was performed after $24 \mathrm{hr}$. Cells were fixed, washed in PBS, and permeabilized. After blocking of endogenous peroxidase activity, cells were incubated with biotinylated anti-BrdU antibody, washed in PBS, followed by incubation with Streptavidin-HRP. The samples were then subjected to BAD substrate for 5 min to develop the desired colour intensity. After washing, cells were stained for A2B5 antibody as an oligodendrocyte progenitors marker. BrdU incorporation was defined as the percentage of A2B5-positive cells 
that were positive for BrdU.

\subsubsection{RhoA activity measurements}

RhoA activity was evaluated by affinity precipitation of active Rho (GTP-bound). Oligodendroglial cells were cultured for 4 days and RhoA activity was measured using Rho assay reagent. Cells were lysed $\left(\mathrm{Mg}^{2+}\right.$ lysis/wash buffer with "Complete" protease inhibitor cocktails) according to the manufacturer's instructions. After removing of the cell debris, the lysates were incubated with Rho assay reagent, which specifically binds to the active form of Rho (Rho-GTP), for 45 min at $4{ }^{\circ} \mathrm{C}$. Beads were then washed 3 times with $\mathrm{Mg}^{2+}$ lysis/wash buffer and once with PBS. The final pellets were then resuspended in sample buffer, boiled for $10 \mathrm{~min}$, and subjected to $12 \%$ SDS-PAGE for western blot analysis using anti-RhoA antibody.

\subsubsection{Protein biochemistry}

\subsubsection{Preparation of cell lysates}

Oligodendrocytes were cultured in PLL-coated dishes for few days. After removing the culture medium, cells were washed with PBS and scraped on ice into lysis buffer $(2 \%$ NP40, 0.2\% SDS, 1 mM EGTA in PBS supplied with "Complete" protease and phosphatase inhibitor cocktail from Roche) or (100 mM Tris-HCl, pH 7.5, $300 \mathrm{mM} \mathrm{NaCl,} 2 \mathrm{mM}$ EDTA, $2 \%$ Triton X-100 supplied with "Complete" protease and phosphatase inhibitor cocktails). The lysates were centrifuged at 10,000 g for $10 \mathrm{~min}$. The protein concentration was determined using Bradford assay. A fraction of the supernatant was mixed with the same amount of sample buffer (20\% glycerol, $4 \mathrm{mM}$ EDTA, 4\% SDS, 4\% 2-mercaptoethanol, $0.05 \%$ Bromophenol blue and $100 \mathrm{mM}$ Tris- $\mathrm{HCl} \mathrm{pH}$ 6.8) and kept at $-20{ }^{\circ} \mathrm{C}$ for further analysis. For p-SKF, Fyn and FAK analysis, cells were lysed using the RIPA buffer (10 mM Tris-HCl pH 7.4, $100 \mathrm{mM} \mathrm{NaCl}, 1 \mathrm{mM}$ EDTA, 1 mM EGTA, $1 \mathrm{mM} \mathrm{NaF}, 20 \mathrm{mM} \mathrm{Na} \mathrm{P}_{2} \mathrm{O}_{4}$, $2 \mathrm{mM} \mathrm{Na} \mathrm{VO}_{4}, 0.1 \%$ SDS, $0.5 \%$ Sodium deoxycholate, $1 \%$ Triton X-100 supplied with "Complete" protease and phosphatase inhibitor cocktails). 


\subsubsection{SDS-PAGE}

Sodium dodecyl sulfate ployacrylamide gel electrophoresis (SDS-PAGE) was performed under denaturing condition to analyse proteins expression and modification. Using a BioRad Mini-PROTEAN 3 casting system, a two-layer (resolving and stacking) gel was set up with the folowing components:

\section{Stacking gel}

4\% Acrylamide/bis-acrylamide (37.5:1) solution

$125 \mathrm{mM} \quad$ Tris-HCl pH 6.8

$0.1 \%$ SDS

$0.05 \%$ Ammonium persulfate (APS)

$0.005 \% \quad(\mathrm{v} / \mathrm{v}) \mathrm{N}, \mathrm{N}, \mathrm{N}^{\prime}, \mathrm{N}^{\prime}-$ Tetramethylethylene-diamine (TEMED)

\section{Resolving gel (8-15\%)}

8-12\% Acrylamide/bis-acrylamide (37.5:1,w:w) solution

$325 \mathrm{mM}$ Tris- $\mathrm{HCl} \mathrm{pH} 6.8$

$0.1 \%$ SDS

$0.05 \%$ APS

$0.005 \% \quad(\mathrm{v} / \mathrm{v})$ TEMED

Separation of protein samples was performed in vertical electrophoresis systems in Trisglycine electrophoresis buffer (25 mM Tris, $192 \mathrm{mM}$ glycine, $0.1 \%$ SDS) for $1 \mathrm{hr}$ at 100 V.

\subsubsection{Western Blot}

After separation with SDS-polyacrylamide gels, the proteins were transferred to a nitrocellulose membrane (Whatman GmbH, Dassel, Germany) using the Bio-Rad Mini-Protein System. The transfer procedure was carried out for $1 \mathrm{hr}$ at $100 \mathrm{~V}$ in a transfer buffer (25 mM Tris, $192 \mathrm{mM}$ glycine, 20\% methanol). After transferring, the membranes were incubated in blocking solution (5\% skim milk powder in PBS) for 30 min at room temperature and afterward incubated with primary antibody in PBST (0.1\% Tween-20 in PBS) at $4{ }^{\circ} \mathrm{C}$ overnight. After washing $3 \times 5$ min at RT with PBST, the membranes were incubated with the appropriate secondary antibodies conjugated to horseradish peroxidase (HRP) for $40 \mathrm{~min}$ at RT, and washed again $3 \times 5 \mathrm{~min}$ at RT with PBST. The proteins 
were detected by enhanced chemiluminescence (Pierce/Thermo Scientific) and bands were quantified using ImageJ Software.

\subsubsection{Myelin membrane analysis}

\subsubsection{Myelin preparation}

The Preparation of myelin membrane was performed as described (Larocca and Norton, $2007)$ previously. The brains from 2 to 6 -month-old mice were homogenized in $0.32 \mathrm{M}$ sucrose solution (containing $5 \mathrm{mM}$ EDTA, $10 \mathrm{mM}$ HEPES pH 7.4 and protease inhibitors) by tip sonication. The homogenates were applied to a two-step sucrose gradients $(0.32 \mathrm{M}$ and 0.85 $\mathrm{M}$ sucrose in HEPES-EDTA buffer). The gradients were centrifuged at 74,000 $\times$ $\mathrm{g}$ for $30 \mathrm{~min}$, and the interfaces (crude myelin) were collected, diluted with $\mathrm{H}_{2} \mathrm{O}$ and centrifuged at 74,000 $\times \mathrm{g}$ for $15 \mathrm{~min}$. The pellet fraction was washed twice with ice-cold $\mathrm{H}_{2} \mathrm{O}$ and recovered by $10 \mathrm{~min}$ centrifugation at $12,500 \times \mathrm{g}$ and the procedure was repeated further to obtain purified myelin. The pure myelin fraction was resuspended in HEPESEDTA buffer and stored at $-20{ }^{\circ} \mathrm{C}$ for further analysis.

\subsubsection{Myelin particles preparation}

Myelin particles were purified using ConA glycoprotein isolation kit. A fraction of myelin membrane was washed and resuspended with $\mathrm{H}_{2} \mathrm{O}$. After passing through 27-gauge needle and sonication for $3 \mathrm{~min}$, the myelin membrane was then applied to ConA beads for 30 min at RT. The flow through was collected using centrifugation at $1,000 \times \mathrm{g}$ for $1 \mathrm{~min}$. To remove MBP from particles, they were resuspended in the following buffers with ratio 2:1; $10 \mathrm{mM}$ Tris-HCl pH 7.4 and $450 \mathrm{mM}$ Tris- $\mathrm{HCl} \mathrm{pH}$ 7.4, $6 \mathrm{mM} \mathrm{CaCl}_{2}, 6 \mathrm{mM} \mathrm{MgCl}_{2}$, for $3 \mathrm{hr}$ at $37^{\circ} \mathrm{C}$ with gentle shaking (Glynn et al., 1987; Earl et al., 1988). The final pellet was resuspended in PBS and kept at $4{ }^{\circ} \mathrm{C}$ for further analysis.

\subsubsection{Myelin particles labelling and binding assay}

Purified myelin particles were fluorescent labeled using two lipophilic dyes (PKH26; red and PKH67; green). Breifly, $100 \mu \mathrm{l}$ of myelin particles were mixed with $900 \mu \mathrm{l}$ of diluent $\mathrm{C}$ and $1 \mu \mathrm{l}$ of PKH dyes. The mixture was vigorously vortexed and incubated for $5 \mathrm{~min}$ at RT. $200 \mu \mathrm{l}$ of FCS was added to the samples to stop the reaction and the mixture was 


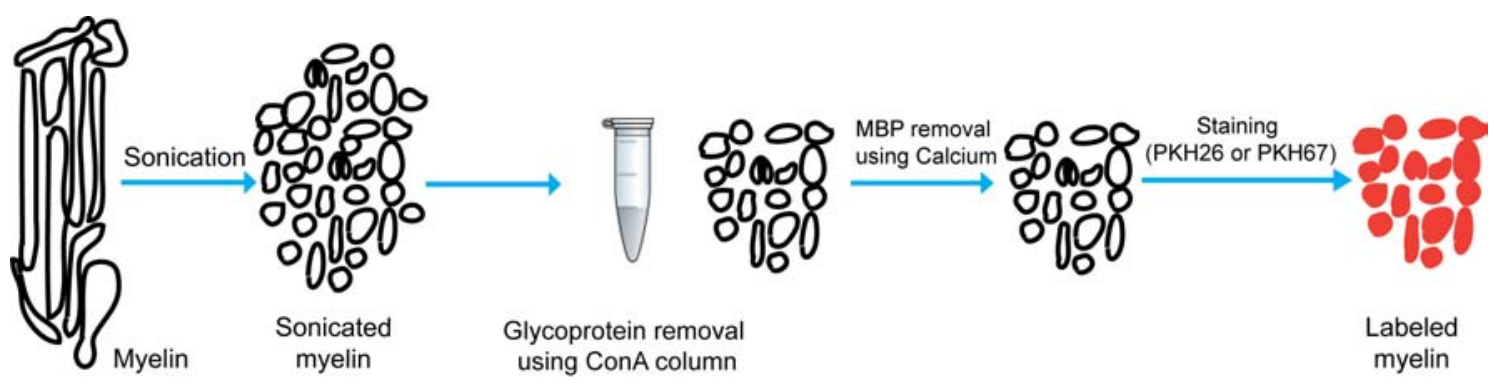

Figure 2.1: Myelin particle preparation and labeling. Preparation and labeling of particle from purified myelin membrane is presented.

applied to centrifugation for $10 \mathrm{~min}$ followed by washing twice with PBS. The final pellet was resuspended in the cell medium and kept at $4{ }^{\circ} \mathrm{C}$ (Figure 2.1).

For binding assay, $100 \mu \mathrm{l}$ of labelled particles were added on top of the cells cultured on coverslips. The binding procedure was carried out for $30 \mathrm{~min}$ at $37^{\circ} \mathrm{C}$ and the cells were directly fixed and processed for immunolabeling. Using confocal imaging the number of myelin particles per cell area was calculated.

\subsubsection{Lectin staining}

All the lectins were obtained from Vector laboratories. Fixed cells were incubated with 5-10 $\mu \mathrm{g} / \mathrm{ml}$ lectins for $10 \mathrm{~min}$ at RT and then mounted on glass slides. For MAL II lectin staining, cells were incubated with $10 \mu \mathrm{g} / \mathrm{ml}$ biotinylated MAL II for $30 \mathrm{~min}$ at RT, followed by washing with PBS and incubating with fluorescent-streptavidine for $10 \mathrm{~min}$ before mounting.

Brain sections prepared by free-floating approach were incubated with $15 \mu \mathrm{g} / \mathrm{ml}$ MAL II at $4{ }^{\circ} \mathrm{C}$ overnight. The samples were then stained by fluorescent-streptavidine for $5 \mathrm{hr}$ at RT and mounted. For WGA staining, sections were incubated with $10 \mu \mathrm{g} / \mathrm{ml}$ fluorescentlectin for $5 \mathrm{hr}$ at RT before mounting. The brain slices were also co-stained with antibody against MOG to label myelinated areas.

\subsubsection{Neuraminidase (sialidase) treatment}

Neuraminidase (Sigma) from Clostridium perfringens was dissolved in PBS pH 6.5 containing $0.05 \%$ BSA (Finkelstein et al., 2007). $5 \mathrm{U} / \mathrm{ml}$ enzyme was added to cells for $1 \mathrm{hr}$ 
at $37{ }^{\circ} \mathrm{C}$ before performing particles binding assay. The efficiency of sialic acid removal by sialidase was assessed using WGA lectin staining.

\subsubsection{Click-iT glycoprotein labeling and detection}

Tetraacetylated azido-mannosamine (Ac4ManNAz) was used to measure the synthesis of sialic acid. Cells were incubated with $50 \mu \mathrm{M}$ Ac4ManNAz for $24 \mathrm{hr}$ at $37^{\circ} \mathrm{C}$. The azidomannosamine was metabolically incorporated into glycoproteins and glycolipids through the permissive feature of the oligosaccharide synthesis. The methabolically incorporation of modified sugar into sialic acid biosynthesis was detected using fluorophore-conjugated alkyne which specifically interacts with the azido-sugar. The amount of sialic acid incorporation into cells was analyzed using ImageJ software.

\subsubsection{Exogenous gangliosides incorporation}

Stock solution of total brain gangliosides was prepared in PBS containing $7 \mathrm{mM}$ defatted BSA. $12 \mu \mathrm{l}$ of solution was loaded to $1 \mathrm{ml}$ culture medium to obtain $80 \mu \mathrm{M}$ concentration of gangliosides and BSA. After incubating for $1 \mathrm{hr}$ at $37^{\circ} \mathrm{C}$, cells were washed extensively with PBS containing $2 \mathrm{mg} / \mathrm{ml}$ fatty acid free-BSA to remove excess lipids (Schwarzmann et al., 1995; Simons et al., 1999). The cells were immediately used for myelin particles binding assay.

\subsubsection{Lipid analysis}

\subsubsection{Lipid extraction}

Lipids from myelin membrane were extracted using chloroform-methanol approach (Bligh and Dyer, 1959). $350 \mu \mathrm{l}$ of myelin was added to $1050 \mu \mathrm{l}$ chloroform-methanol (ratio 2:1). The mixture was then vortexed vigorously and centrifuged at 6,000 rpm for $5 \mathrm{~min}$ at RT. The organic phase (lower fraction) was recovered and added to $70 \mu \mathrm{l}$ methanol and $475 \mu \mathrm{l}$ $50 \mathrm{mM} \mathrm{NaCl}$ followed by mixing and centrifuging at 6,000 rpm for $5 \mathrm{~min}$. The lower organic phase containing lipids was collected and applied to speed vacuum for 45 min at $37^{\circ} \mathrm{C}$ to obtain a dry lipid fraction. 


\subsubsection{Liposome preparation and aggregation}

Liposomes from myelin lipids with or without exogenous lipids were prepared as described previously (Boggs et al., 1994; Ter Beest et al., 1994). Lipids (including proteolipid proteins), extracted from myelin membrane, were dissolved in 2-chloroethanol to obtain the total concentration of $1 \mathrm{mg} / \mathrm{ml}$ lipid/protein. The protein/lipid suspensions were briefly centrifuged and dialyzed at RT for $6 \mathrm{hr}$ against 2 litres of reconstitution buffer $(5 \mathrm{mM}$ HEPES, $1 \mathrm{mM}$ EDTA, and $10 \mathrm{mM} \mathrm{NaCl}$, pH 5.0) using Slide-A-Lyzer MINI dialysis device (Thermo scientific, Schwerte, Germany) with cut-off approximately $10 \mathrm{kDa}$. After three times changing of dialysis buffer, the samples were collected and analyzed. To measure liposome aggregation, the optical density of samples was monitored at $405 \mathrm{~nm}$ using a Biophotometer Plus (Eppendorf) (Johnson and Chapman, 2010).

\subsubsection{Dot blot}

In order to immunolable the lipids in myelin liposomes, dot blot assay was performed. $8 \mu \mathrm{l}$ of liposomes was applied and dried into a nitrocellulose membrane. The membrane was then incubated in blocking solution (5\% skim milk powder in PBS) for 30 min at RT and incubated with primary antibody in PBS at $4{ }^{\circ} \mathrm{C}$ overnight. After washing with PBS, the membrane was incubated with the appropriate HRP-secondary antibodies for $40 \mathrm{~min}$ at $\mathrm{RT}$ and the corresponded lipids were detected by enhanced chemiluminescence.

\subsubsection{Immunocytochemistry and imaging}

\subsubsection{Immunocytochemistry}

For immunoflourescence, cells were fixed with $4 \%$ paraformaldehyde (PFA) for 15 min at RT and were permeabilized with $0.1 \%$ Triton X-100 in PBS for 1 min. Fixed cells were then incubated with blocking solution ( $2 \%$ BSA, $0.2 \%$ Fish gelatine, and $2 \%$ FSC in PBS) for $30 \mathrm{~min}$ at room temperature. Afterward, cells were incubated with primary antibodies diluted in blocking solution for $1 \mathrm{hr}$, followed by three washing steps in PBS and then incubated with secondary antibodies for another $1 \mathrm{hr}$. The glass coverslips were finally mounted onto a glass slide with a drop of mowiol solution (2.4 g Mowiol, 6 g Glycerol, $6 \mathrm{~mL} \mathrm{H} \mathrm{H}_{2} \mathrm{O}, 12 \mathrm{~mL} 0.2 \mathrm{M}$ Tris- $\mathrm{HCl} \mathrm{pH} 8.5$ ) and dried overnight in the dark condition. 


\subsubsection{Confocal Microscopy}

The morphological analysis of fixed cell samples was performed using laser scanning confocal microscopy. Fluorescence images were acquired with Leica DMIRE2 microscope and a Leica TCS SP2 AOBS confocal laser scanning setup (Leica Microsystems, Mannheim, Germany).

\subsubsection{Image Analysis}

Quantification of the cell surface area of individual cells was approximated by measuring the projected area (the area projected by a single cell onto a single optical plane) using Meta Imaging software Series 6.1.

Quantification of myelin particles binding to cell surface area was assessed using ImageJ and Leica Confocal software, 2.61.

Quantification of fluorescence intensities between different confocal images was performed using ImageJ software. The mean intensity of fluorescent signal from different cell areas was calculated and analyzed.

\subsubsection{Atomic Force Microscopy (Single particle force spectroscopy)}

Gold-coated cantilevers (Arrow TL2Au, Nanoworld) were used for single particle force spectroscopy measurements. The cantilevers were washed twice in isopropanol and ultrapure water for 1 min each. Thereafter, cantilevers were cleaned using a plasma cleaner for $1 \mathrm{~min}$. For binding of the myelin particles, the cantilevers were incubated in a $10 \mu \mathrm{g} / \mathrm{mL}$ poly-D-lysine solution for $10 \mathrm{~min}$ and washed in ultra-pure water afterwards. Measurements were performed using a CellHesion 200 setup (JPK Instruments, Berlin, Germany) combined with an Olympus IX81 microscope (Hamburg, Germany) and a petridish heater assuring a constant temperature of $37{ }^{\circ} \mathrm{C}$ throughout the measurement. A contact time of $30 \mathrm{sec}$ and a force of $500 \mathrm{pN}$ were used to bind the particles to the poly-D-lysine coated cantilever. For investigation of the myelin-oligodendrocytes interaction, the time of particle-cell contact (5 sec), the force of approach $(500 \mathrm{pN})$ and the velocity of cantilever retraction $(5 \mu \mathrm{m} / \mathrm{s})$ were kept constant throughout the measurements. To quantify 
the interaction between myelin particles and oligodendrocytes, maximum adhesion force (minimum in force during cantilever retraction) from the recorded force retraction curves was analyzed. To evaluate the statistics of the obtained data, a non-parametric statistical hypothesis test, namely Wilcoxon rank-sum test, was employed.

\subsubsection{Electron microscopy}

\subsubsection{Conventional embedding}

Sample preparation for electron microscopy analysis was performed as described previously (Möbius et al., 2010). Briefly, the dissected nerves were chemically fixed by immersion fixation in $4 \% \mathrm{PFA}$ and $2.5 \%$ glutaraldehyde for $4 \mathrm{hr}$. The samples were then washed with $0.1 \mathrm{M}$ phosphate buffer, postfixed with $2 \% \mathrm{OsO}_{4}$ for $4 \mathrm{hr}$ at $4{ }^{\circ} \mathrm{C}$. They were afterward dehydrated in a graded series of ethanol followed by propylene oxide and were infiltrated with Epon-propylene oxide mixtures and pure Epon. The nerves were finally transferred into embedding molds and polymerized for $24 \mathrm{hr}$ at $60{ }^{\circ} \mathrm{C}$.

\subsubsection{High pressure freezing}

The high pressure-freezing (HPF) of freshly extracted optic nerves was performed with HPM100 (Leica, Vienna, Austria). The samples were placed in a $2 \mathrm{~mm}$ inner diameter specimen carrier with $0.2 \mathrm{~mm}$ depth (Microscopy Services, Flintbek, Germany) surrounded by $20 \%$ PVP (polyvinylpyrrolidone) in PBS to exclude the air inclusion around the samples and then frozen at 2000 bar for few milliseconds (Möbius et al., 2010).

\subsubsection{Freeze substitution and embedding}

Freeze substitution was carried out for high-pressure frozen samples using a Leica AFS2 unit (Leica, Vienna, Austria). First the samples were placed in tannic acid $(0.1 \%$ in acetone) for $100 \mathrm{hr}$ at $-90{ }^{\circ} \mathrm{C}$, then washed with acetone at $-90{ }^{\circ} \mathrm{C}$ and subsequently incubated with new substitution solution containing $\mathrm{OsO}_{4}\left(2 \% \mathrm{OsO}_{4}\right.$ in acetone, $\left.-90{ }^{\circ} \mathrm{C}\right)$ and $0.1 \%$ uranyl acetate. Afterward the temperature was increased from $-90{ }^{\circ} \mathrm{C}$ to $-20{ }^{\circ} \mathrm{C}$ in increments of $5{ }^{\circ} \mathrm{C} / \mathrm{hr}$, and the samples were kept for $16 \mathrm{hr}$ at $-20{ }^{\circ} \mathrm{C}$. The temperature was raised further from $-20{ }^{\circ} \mathrm{C}$ to $+4{ }^{\circ} \mathrm{C}$ in increments of $10{ }^{\circ} \mathrm{C} / \mathrm{hr}$. The samples were 
next washed with acetone at $4{ }^{\circ} \mathrm{C}$, allowed to adjust to room temperature for $1 \mathrm{hr}$ and were then transferred into Epon (50\% Epon in acetone for $3 \mathrm{hr}$, 90\% Epon in acetone overnight, and 100\% Epon for $6 \mathrm{hr}$ ). Finally, the samples were placed in embedding molds and polymerized for $24 \mathrm{hr}$ at $60{ }^{\circ} \mathrm{C}$ (Möbius et al., 2010).

\subsubsection{Sectioning and electron microscopy}

The ultrathin sections for imaging were prepared using a Leica Ultracut S ultramicrotome set and sections with $50 \mathrm{~nm}$ thick were cut. The sections were incubated in $2 \%$ aqueous uranyl acetate for $30 \mathrm{~min}$ for contrasting, and then washed in water followed by lead citrate for 5 min and again washed in water and dried. The imaging was performed using an electron microscope LEO EM 912 AB (Zeiss, Oberkochen, Germany) used at $80 \mathrm{kV}$ and images recorded with a CCD camera 2048 x2048 (Proscan, Scheuring, Germany) (Möbius et al., 2010).

Negative staining electron microscopy was performed as described (Trajkovic et al., 2008). 


\title{
Chapter 3
}

\section{Results}

\subsection{The function of oligodendrocyte-derived exosome-like vesicles in central nervous system}

Most of the results in Section 3.1 have been published in:

\author{
Inhibition of myelin membrane sheath formation by oligodendrocyte-derived \\ exosome-like vesicles \\ Mostafa Bakhti, Christine Winter and Mikael Simons \\ J. Biol. Chem. 286(1):787-796 (2011)
}

\subsubsection{Characterization of oligodendrocyte-derived exosomes}

Exosomes from rat oligodendrocytes were collected using differential centrifugation from the medium of cultured cells between 6 to 8 days after shaking. The amount of PLP was evaluated in the different centrifugation steps to confirm the enrichment of this protein in the exosomes fraction (P100) (Figure $3.1 \mathrm{~A}$ ). Next, we explored the exosomes fraction and cell lysate for different protein markers using western blot analysis. PLP, CNPase, MAG and MOG were found as positive control proteins in exosomes, whereas there was no signal for calnexin and Nogo A, considered as the negative markers to control the presence of oligodendrocytes endoplasmic reticulum (ER) (Figure 3.1 B). The secretion of exosomes by oligodendrocytes was also evaluated by electron microscopy. Negative staining of oligodendrocytes-derived exosomes exhibited the presence of 30 to $100 \mathrm{~nm}$ vesicles corresponding to exosome structures (Figure 3.2). 
A

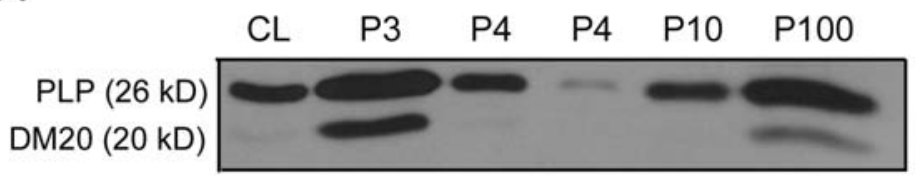

B
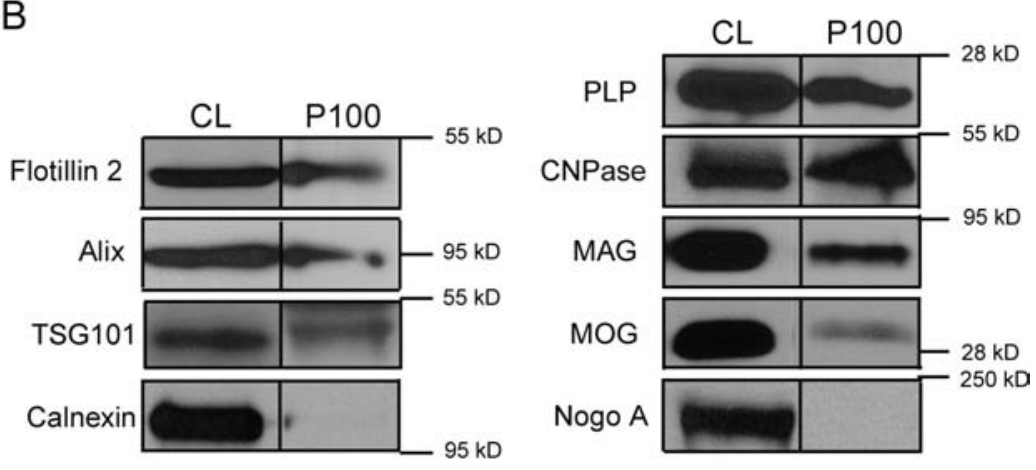

Figure 3.1: Characterization of oligodendrocyte-derived exosomes. (A) A 6 to 8-day-old medium from OLs was collected and submitted to different centrifugation steps as indicated. The resulting pellets of each centrifugation step were analyzed by Western blotting for PLP. (B) Cell lysate (CL) and exosomes (P100) were analyzed for different markers.

\subsubsection{The effect of exosomes on myelination}

To define the function of OL exosomes, their effect on the myelination process was examined in cell culture system. We used a primary oligodendrocyte-mixed brain co-culture to assess the role of exosomes on myelination. Exogenous exosomes $(0.1 \mathrm{\mu g} / \mathrm{ml})$ were added to one-day-old-co-culture and myelination was monitored after 4 days. MBP and $\beta$-III-tubulin were used as the markers of oligodendrocytes and neurons, respectively. Surprisingly, we found that exosomes significantly inhibited myelination in co-culture model system (Figure $3.3 \mathrm{~A}$ ). As shown in figure $3.3 \mathrm{~B}$ the number of MBP positive cells with parallel structures are decreased in exosomes-treated sample as compared to the control. These MBP positive cells with parallel structures have been previously described as myelinating OLs (Stevens et al., 2002; Laursen et al., 2009). 


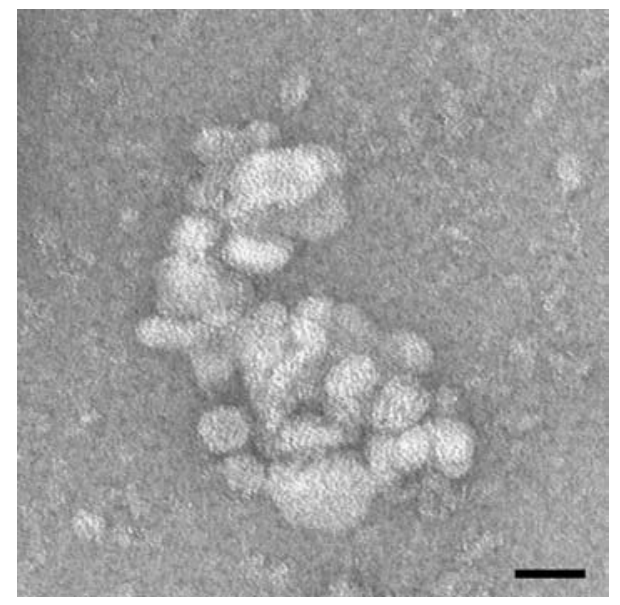

Figure 3.2: Electron microscopic of oligodendrocyte-derived exosomes. Electron microscopic negative staining of P100 $(100,000 \times \mathrm{g})$ pellet showed the presence of 30 to $100 \mathrm{~nm}$ vesicles. Scale bar, $50 \mathrm{~nm}$.

A

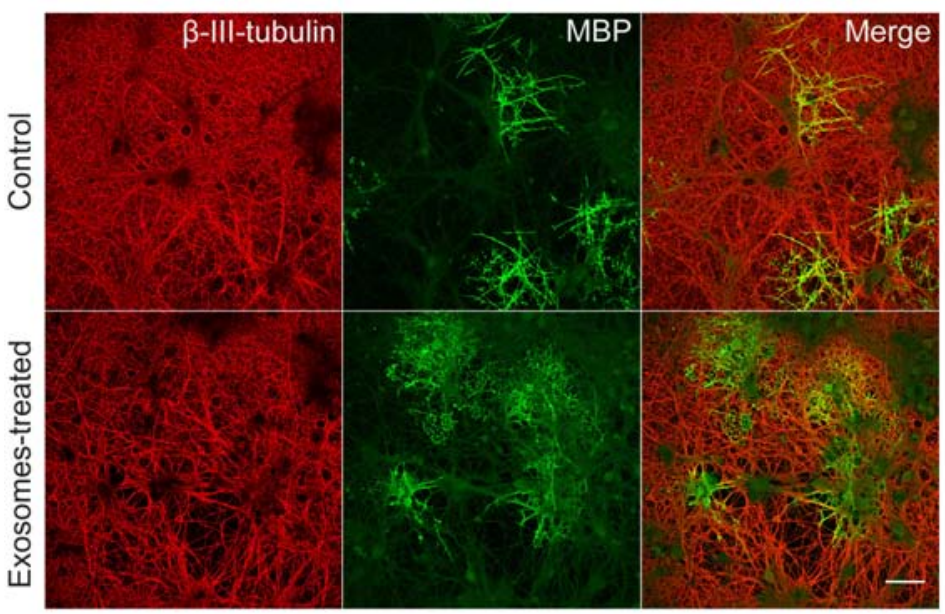

B
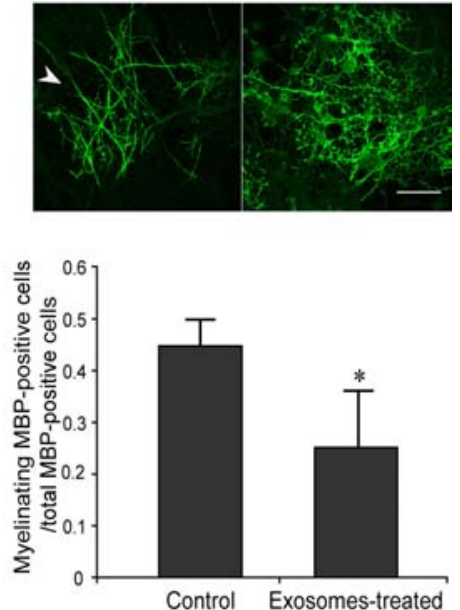

Figure 3.3: Exosomes inhibit myelination. (A) OLs-neurons coculture was treated with exosomes for 4 days. The cells were immunolabled for $\beta$-III-tubulin (red) and MBP (green). Scale bar, $100 \mu \mathrm{m}$. (B) ) The level of myelination was measured by counting the number of MBP cells with multiple parallel processes (arrowhead) as a percentage of total MBP-positive cells $\left(n=3,{ }^{*} p\right.$ $<0.05$; $t$-test). Scale bar, $50 \mu \mathrm{m}$. 


\subsubsection{Effect of neuronal signals on exosome release by primary oligodendro- cytes}

After observing a regulatory role of exosomes in myelination process, we investigated whether the secretion of exosomes was influenced by neuronal signals. It has been shown that the treatment of oligodendroglial cells with conditioned medium from mixed brain cultures (NCM) reduces the late endosomal/lyososomal pool of PLP (Trajkovic et al., 2006). Therefore, a 14-day-old conditioned medium from mixed brain culture was used as the source of neuronal signals. The medium of OLs on day 6 was substituted by fresh medium or neuronal conditioned medium (NCM) and exosome release was monitored after $24 \mathrm{hr}$ by measuring the amount of PLP in P100 fraction. Treatment of OLs by NCM resulted in a significant decrease of exosome production by these cells (Figure 3.4 A). More than 60 percent reduction in exosomal PLP level was detected during treatment of OLs by NCM as compared to the control. Neuronal conditioned medium also reduced the secretion of CNPase and Alix (Figure 3.4 B).

A
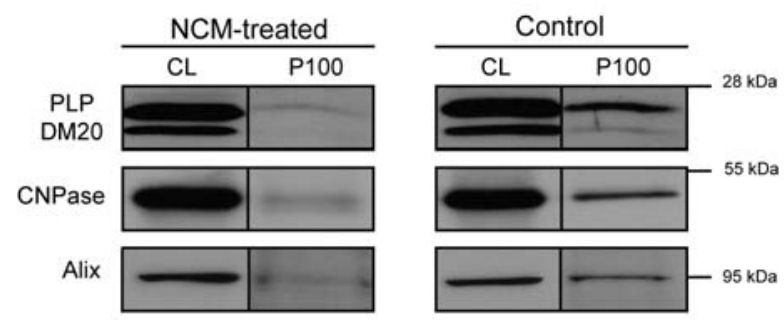

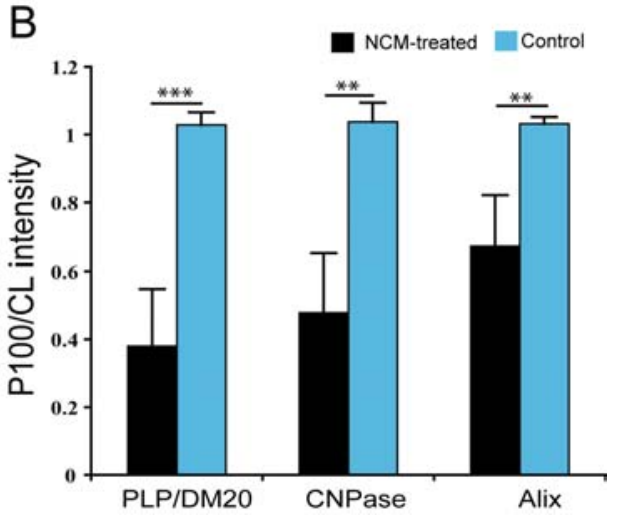

Figure 3.4: Neuronal conditioned medium reduces exosome release by OLs. (A) The medium of OLs in day 6 was substituted by NCM and the exosomes preparation was performed after $24 \mathrm{hr}$. (B) The amount of PLP, CNPase, and Alix from cell lysate and exosomes fraction was quantified $\left(\mathrm{n}=3,{ }^{* *} p<0.01 ;{ }^{* * *} p<0.001 ; t\right.$-test $)$.

\subsubsection{Effect of OL-derived exosomes on oligodendrocytes}

We found that exosomes secreted by OLs inhibits myelination. Since the presence of neurons reduced exosomes release by OLs, we proposed that exosomes may have some 
A

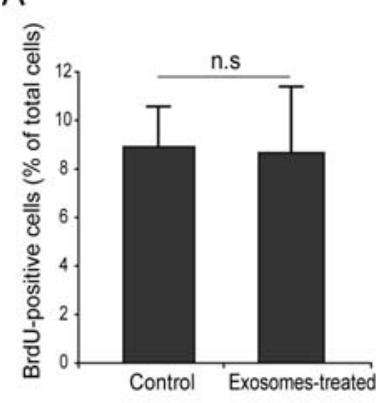

B

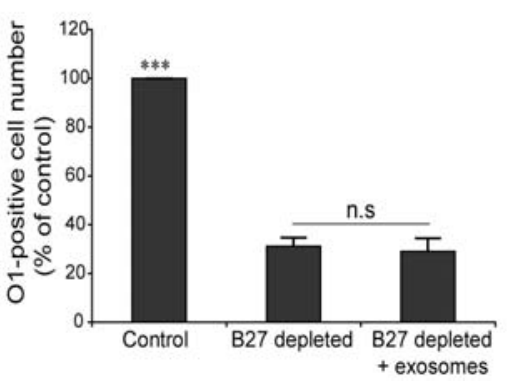

C

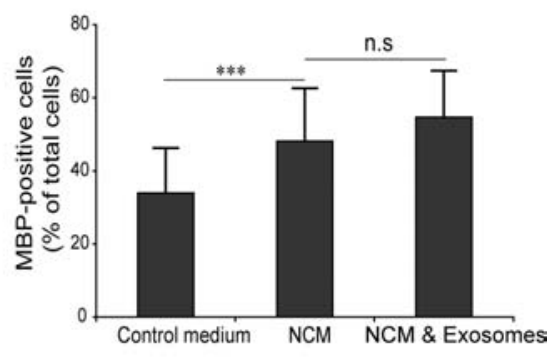

Figure 3.5: Exosome effects on the proliferation, viability, and differentiation of oligodendrocytes. (A) Cells were treated by $10 \mu \mathrm{M}$ BrdU after one day of seeding. The proliferation assay was performed after $24 \mathrm{hr}(\mathrm{n}=3)$. (B) B-27 supplement depletion was used to induce OLs cell death. Treatment with exosomes did not prevent oligodendrocytes mortality ( $n=50$ confocal images from two different experiments). (C) Treatment of OLs by NCM for 3 days induced their differentiation, which was measured by increase in the percentage of MBP positive cells. Exosomes did not change this process ( $n=3,{ }^{* * *} p<0.001$; one-way ANOVA).

autocrine effects on oligodendrocytes. Therefore, we assessed the effect of exosomes on different stages of oligodendrocyte development. First, we examined whether exosomes modulate oligodendrocyte proliferation by performing a bromodeoxyuridine (BrdU) assay on freshly plated cells. No significant alteration in BrdU incorporation was observed in exosome-treated oligodendrocytes when compared to the controls (Figure 3.5 A). We next investigated the possible effects of exosome-like vesicles on oligodendrocyte survival by applying a growth factor depletion assay (Laursen et al., 2009). Removal of B-27 supplement induced cell death, which was not affected by the treatment with exosomes (Figure 3.5 B). We also explored the effect of exosomes on oligodendrocyte differentiation. When oligodendrocytes were cultured in normal medium or in NCM and analyzed 2-3 days later, we observed that NCM promoted oligodendrocyte differentiation as detected by an increase in the fraction of MBP-positive cells. Treatment of oligodendrocytes with exosome-like vesicles, however, did not affect this process (Figure 3.5 C). Differentiated oligodendrocytes undergo the final step of their development to produce a large amount of myelin membrane. This stage correlates with expansion of the cell surface area and production of large membrane sheets required for myelin biogenesis. Many different factors have been reported to affect the OL surface branching which subsequently influence their myelinating properties (Rajasekharan et al., 2009; Laursen et al., 2009; Baer et al., 2009). We next 
studied whether the inhibitory effect of exosomes on myelination is by influencing the OL branching. NCM treatment resulted in a relatively robust increase in the size of oligodendrocytes in comparison to the cells kept in unconditioned control medium. This effect was observed in both undifferentiated (A2B5-positive) and differentiated (MBP-positive) cells (Figure 3.6 A). Interestingly, incubation of cells with exosome-like vesicles reversed the effect of NCM on cell surface expansion (Figure 3.6 B).

A

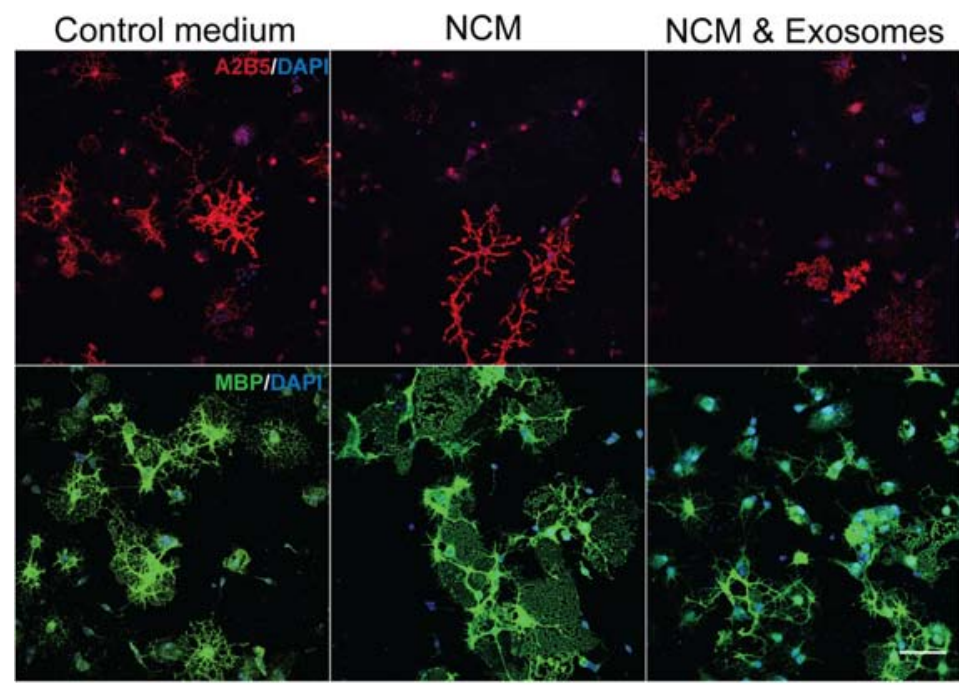

B

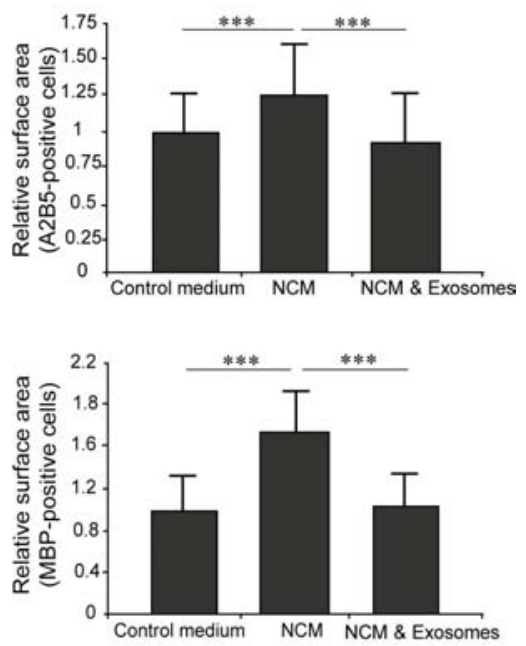

Figure 3.6: Exosomes inhibit cell surface expansion of oligodendrocytes. (A) The effect of NCM and exosomes on OLs morphology is represented. Oligodendrocytes were treated for 3 days with NCM and incubated with exosomes during the last 2 days. Scale bar, $50 \mu \mathrm{m}$. (B) Cell surface area of A2B5- and MBP-positive cells was quantified. NCM treatment enhanced OL cell surface area compared to the controls, which were cultured in the fresh medium. Addition of exosomes prevented the effect of NCM and induced OLs surface retraction ( $n=50$ confocal images from three different experiments, ${ }^{* * *} p<0.001$; one-way ANOVA).

\subsubsection{RhoA pathway involvement in exosome effect on OLs}

Having identified a role of exosome-like vesicles in regulating cell surface size, we investigated the potential signal transduction pathways involved in this process. The mTOR signalling is known to regulate the myelin membrane formation in oligodendrocytes (Tyler et al., 2009; Narayanan et al., 2009). Activation of mTOR signalling results in the phos- 


\section{Figure 3.7: Exosomes do not influnce the mTOR pathway. OLs were cultured for 3 days and subsequently treated with NCM and exosomes as indicated. Cells were an- alyzed by western blotting using specific an- tibodies against phosphorylated Akt (Ser- 473), Akt, phosphorylated ribosomal sub- unit 6 (Ser-236), and actin as the loading control.}

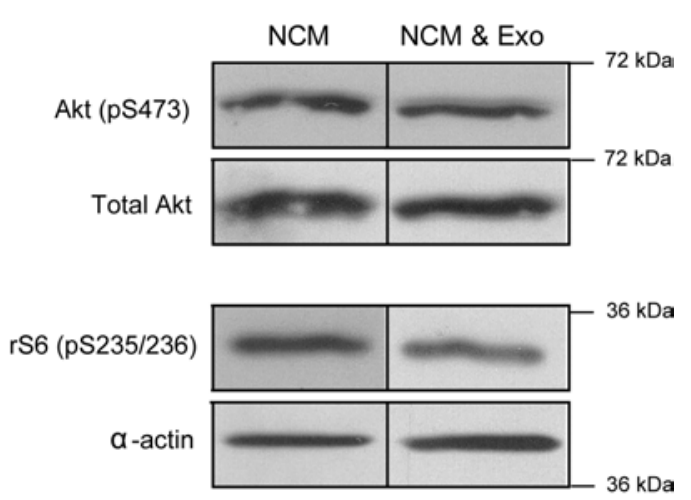

phorylation of Akt and the ribosomal subunit 6 (rS6). However, exosome-like vesicles did not change the phosphorylation status of these proteins when compared to control conditions, which argues against the involvement of this pathway in mediating the changes in cell surface area (Figure 3.7).

Inactivation of RhoA pathway has been described to play a central role in the oligodendrocyte process branching (Liang et al., 2004; Kippert et al., 2007, 2009). Therefore, we explored the involvement of this pathway in exosome induced OLs retraction. Indeed, when we applied the Rho-associated kinase (ROCK) inhibitor Y-27632 (Y27), the inhibitory effect of exosome-like vesicles on cell surface expansion was prohibited (Figure 3.8 A). Similar results were obtained when the myosin-II inhibitor, blebbistatin, was introduced in the experiments (Figure $3.9 \mathrm{~A}$ ). Intriguingly, we found that both blebbistatin and Y27 did not affect cell surface size of oligodendrocytes cultured in NCM in the absence of exosome-like vesicles (Figure 3.8 B, Figure 3.9 B). Taken together, these data indicate that exosome-like vesicles activate the Rho-ROCK-Myosin signalling axis to inhibit cell surface expansion of oligodendrocytes.

To verify the involvement of RhoA pathway, its activity was measured by affinity precipitation of active (GTP-bound) Rho from cell lysates using agarose beads containing the Rho-binding domain of Rhotekin. Pull-down approach revealed that the active form of RhoA (RhoA-GTP) is increased upon treatment of oligodendrocytes with exosomelike vesicles (Figure 3.10 A). To obtain further support for this pathway, we assessed the phosphorylation level of myosin light chain 2 (MLC2), a downstream molecule of RhoAROCK. We found that MLC2 phosphorylation level is elevated when cells were treated 
A

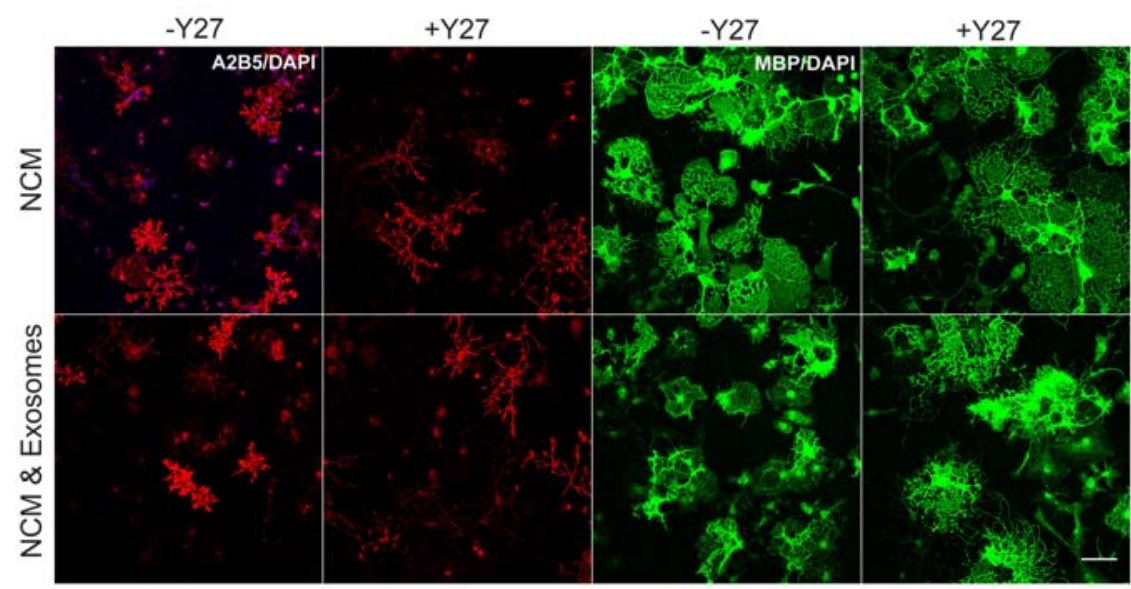

B
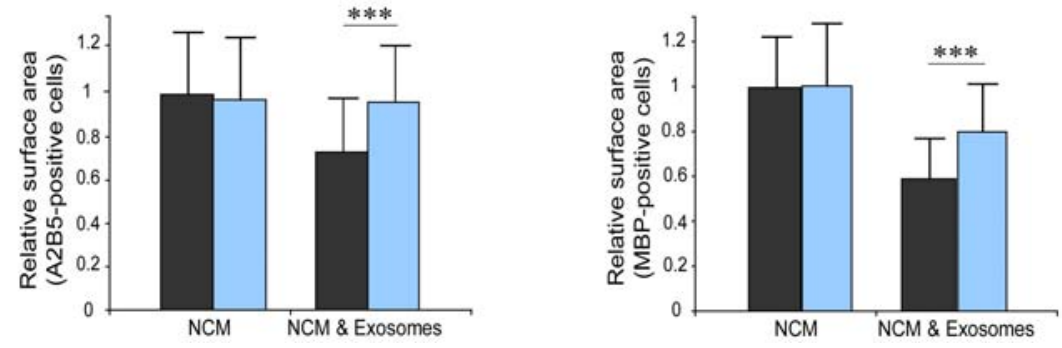

Figure 3.8: Inhibition of ROCK prevents the effect of exosome on cell surface size. (A) Oligodendrocyte cells were treated with NCM and exosomes in the absence or presence of 10 $\mu \mathrm{M}$ Y27632 (Y27) as the ROCK inhibitor. Scale bar, $50 \mu \mathrm{m}$. (B) Y27 did not affect the surface area of cells treated with NCM, but reduced the inhibitory effect of exosomes on cell surface expansion. Black column, without $\mathrm{Y} 27$; blue column, with $\mathrm{Y} 27$. ( $\mathrm{n}=42$ confocal images from three different experiments, ${ }^{* * *} p<0.001 ; t$-test)

with exosome-like vesicles (Figure $3.10 \mathrm{~B}$ ). These data demonstrate that exosome-like vesicles act by influencing actomyosin contractility in oligodendrocytes.

\subsubsection{Fyn and FAK phosphorylation are involved in exosomes effect on OLs}

Fyn is a member of Src family kinases (SFKs), and plays a vital role in myelination of the CNS (Liang et al., 2004). This protein has been shown to affect the differentiation and myelination steps of OLs. Activation of Fyn suppresses RhoA pathway and increases OLs process branching (Goto et al., 2008; Câmara et al., 2009; Laursen et al., 2009; Relucio et al., 2009). To indicate whether NCM increases oligodendrocyte surface area in a Src 


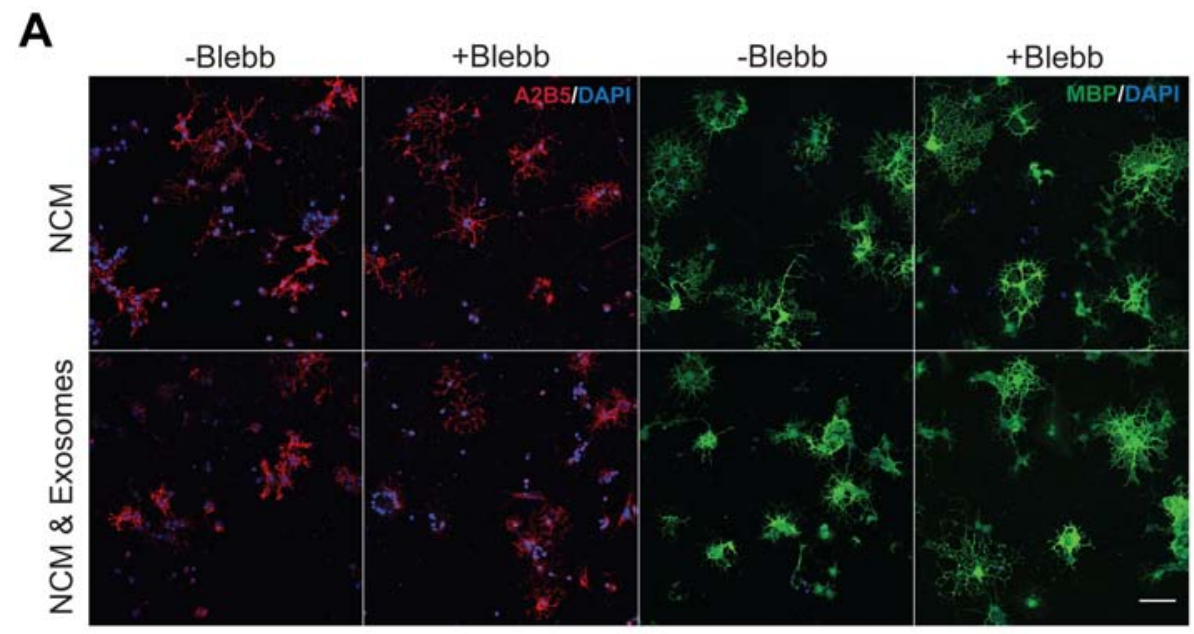

B
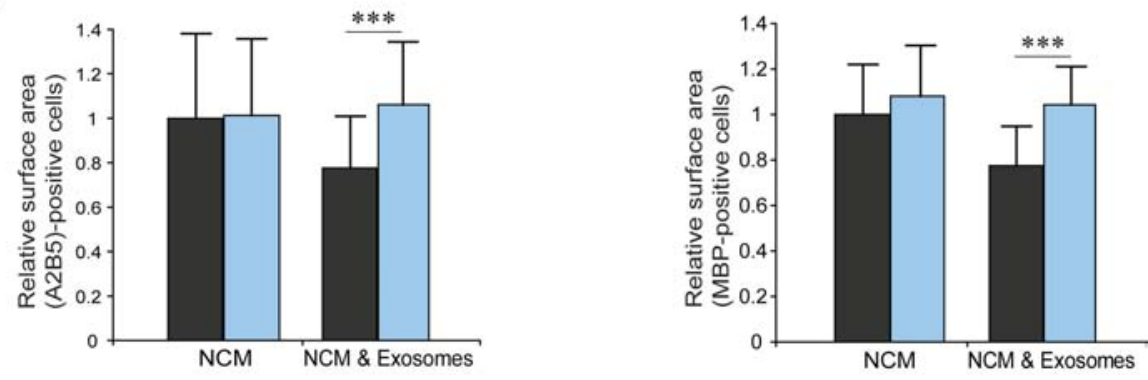

Figure 3.9: Inhibition of myosin-II reduces the effect of exosome on cell surface size. (A) Oligodendrocytes were treated with NCM and exosomes in the absence or presence of $50 \mu \mathrm{M}$ blebbistatin (Blebb) as the myosin-II inhibitor. Scale bar, $50 \mu \mathrm{m}$. (B) Blebb did not influence the surface area of cells treated with NCM, but decreased the inhibitory effect of exosomes on cell surface expansion. Black column, without Blebb; blue column, with Blebb. ( $\mathrm{n}=30$ confocal images from two different experiments, ${ }^{* * *} p<0.001 ; t$-test)

kinase-dependent signalling, we used the small molecular inhibitor, PP2. Treatment of cultured oligodendrocytes with PP2 inhibited the cell surface expansion induced by NCM, confirming involvement of Src proteins (Figure 3.11 A, B). Fyn activity is regulated by two different phosphorylation sites. Phosphorylation of tyrosine 420 and 531 residues lead to activation and inactivation of this protein, respectively. We examined the phosphorylation status of Tyr-420 and Tyr-531 after exosome treatment. Western blot analysis revealed a significant decrease in the activating (Tyr-420), but not the inactivating phosphorylation site (Tyr-531) in cells which had been incubated with exosomes (Figure 3.12 A, B). Fyn signalling is connected to another kinase, the focal adhesion kinase (FAK), which 

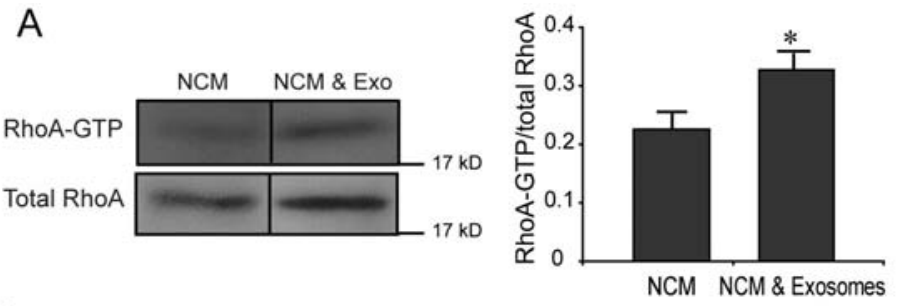

B
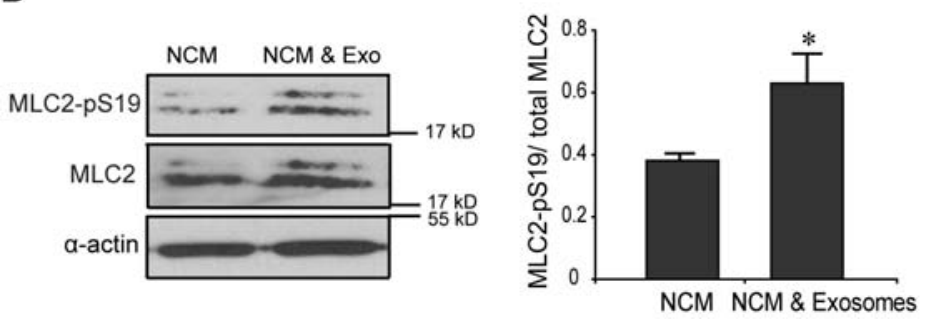

Figure 3.10: Inhibition of actomyosin contractility prevents the effect of exosomes on oligodendrocytes cell surface area. (A) Using a pull down assay, the amount of active RhoA (RhoA-GTP) was measured during treatment of oligodendrocytes with NCM and exosomes. (B) Treated cells were also analyzed for changes in phosphorylation level of myosin light chain 2 (MLC2) protein ( $\mathrm{n}=3,{ }^{*} p<0.05 ; t$-test).

has been reported to modulate CNS myelination (Umemori et al., 1994; Forrest et al., 2009). Integrins and numerous extracellular signals activate FAK, which in turn leads to FAK autophosphorylation at Tyr-397, generating a binding site for the Src homology 2 (SH2) domain of SFKs such as Fyn. We tested the autophosphorylation level of tyrosine 397 residue in FAK and observed a significant decrease in Tyr-397 phosphorylation after exosome treatment (Figure $3.13 \mathrm{~A}, \mathrm{~B}$ ). 
A

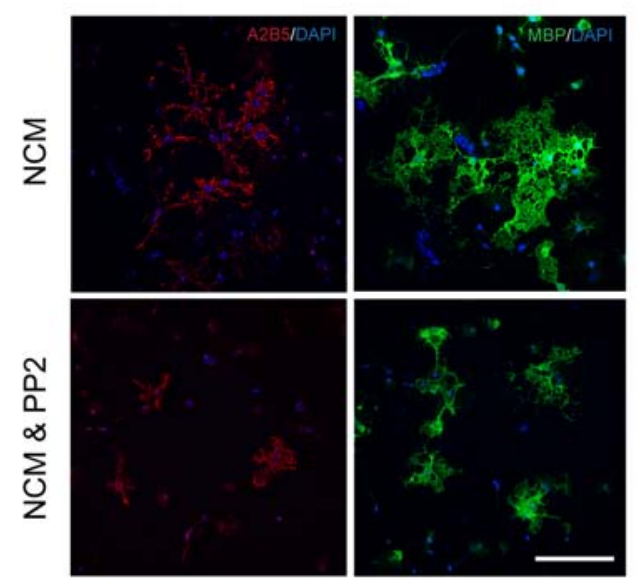

B
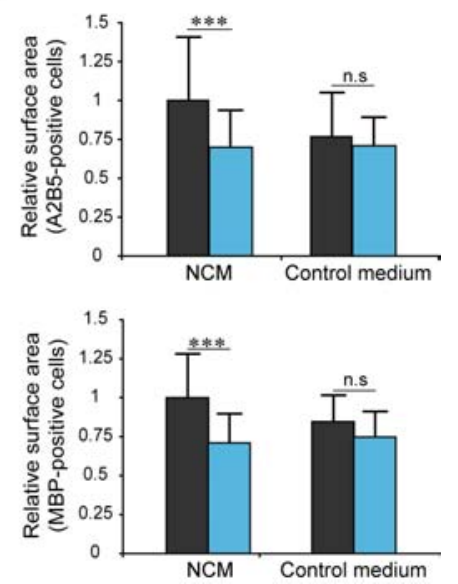

Figure 3.11: NCM increases cell surface area by activation of Src proteins. (A) NCM-treated oligodendrocytes were cultured for 3 days in presence or absence of Src family kinase inhibitor (PP2), for the last 2 days. Scale bar, $100 \mu \mathrm{m}$. (B) While PP2 reduces the surface area of NCMtreated cells, it did not affect cells, cultured in control medium. Black column, without PP2; blue column, with PP2 ( $\mathrm{n}=40$ confocal images from three different experiments, ${ }^{* * *} p<0.001 ; t$-test).

A

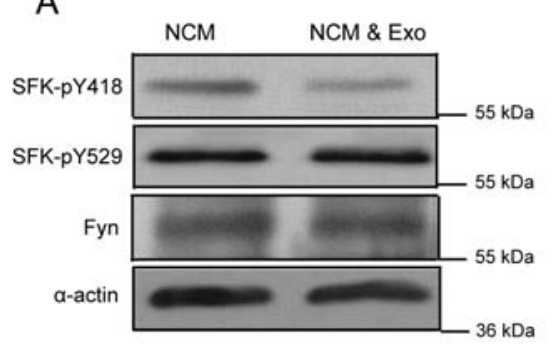

B
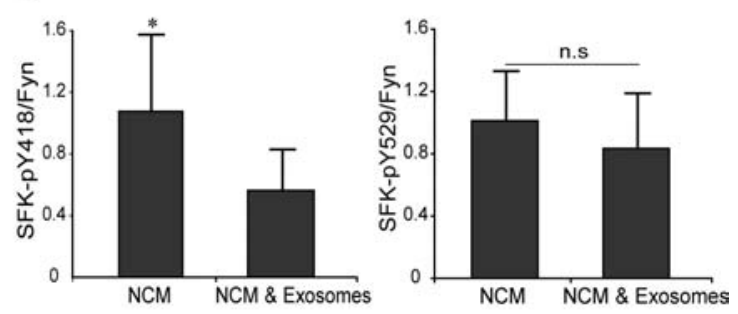

Figure 3.12: Exosomes affect phosphorylation of Fyn protein. (A) Western blot analysis for OLs treated with NCM or exosomes using SFK (pY418), SFK (pY529) and Fyn specific antibodies.

(B) The quantification of 4 independent experiments is presented. ( ${ }^{*} p<0.05 ; t$-test). 
A

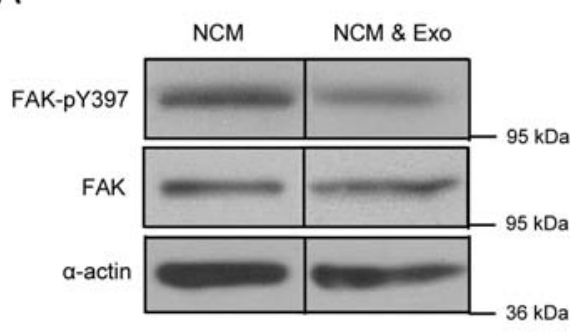

B

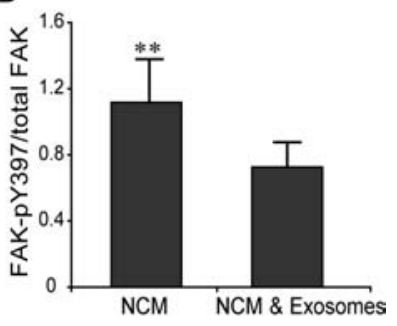

Figure 3.13: Exosomes inhibit autophosphorylation of Focal adhesion kinase (FAK). (A) The autophosphorylation of FAK was evaluated using antibody against phosphorylated tyrosine 397. (B) Phosphorylation level of FAK during OLs treatment with NCM and exosomes from 3 independent experiments is shown. $\left({ }^{* *} p<0.01 ; t\right.$ test).

\subsection{Mechanism of myelin membrane adhesion and compaction in central nervous system}

Most of the results in Section 3.2 have been prepared for publication:

PLP adhesive property and sialic acid removal increase myelin adhesion and stability in the CNS

Mostafa Bakhti, Nicolas Snaidero, David Schneider, Shweta Aggarwal, Wiebke

Möbius, Andreas Janshoff, Matthias Eckhardt, Klaus Armin Nave and Mikael

Simons

Manuscript under preparation

\subsubsection{A new assay to assess myelin membrane adhesion in vitro}

Most of our knowledge about myelin structure is based on an approaches employing electron microscopy and mouse genetic. Due to its tightly packed structure, cell biological mechanisms regulating myelin assembly can not be studied easily by light microscopy. Because of limitations with current approaches, the exact mechanism of myelin compaction remains obscure. It is known that primary oligodendrocytes produce myelin-like mem- 

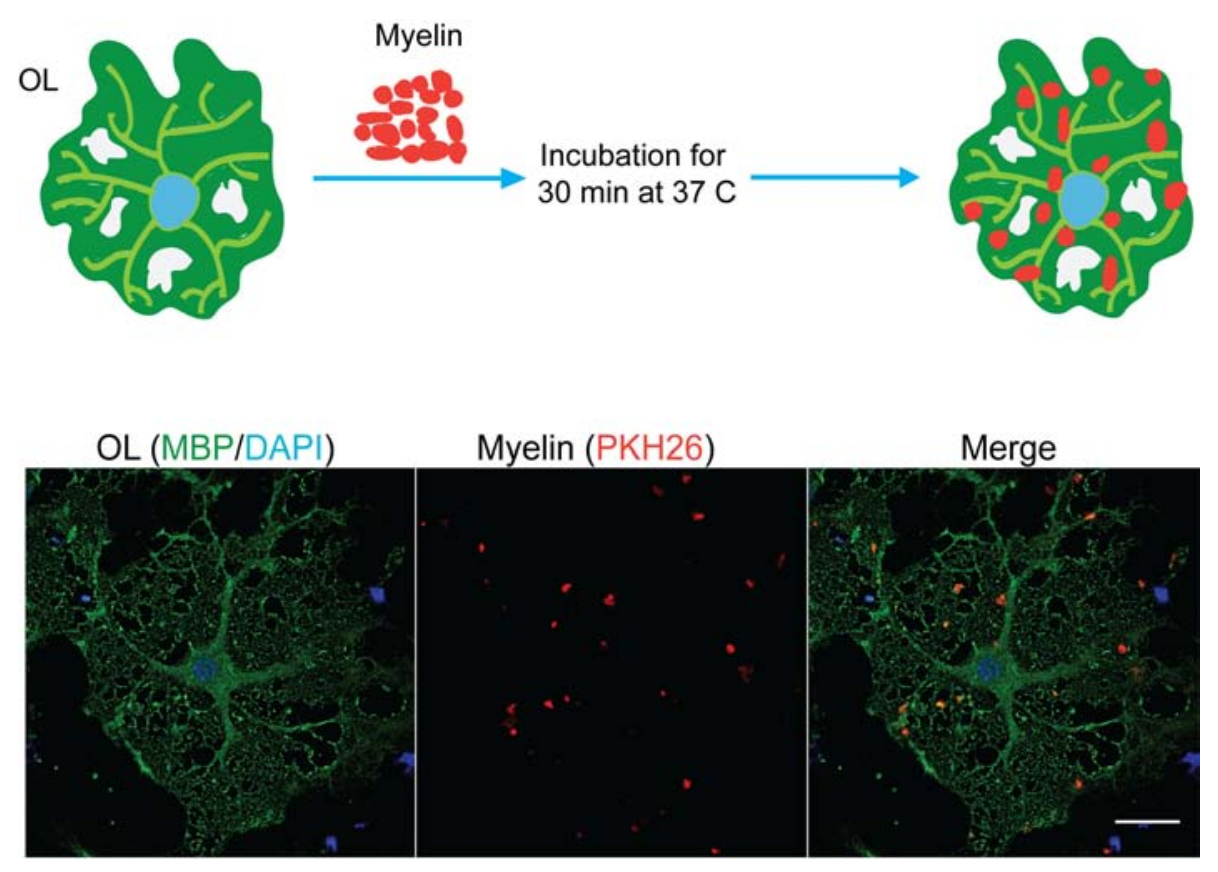

Figure 3.14: Binding assay. The schematic and confocal images of the interaction between labeled myelin particles and primary oligodendrocytes (OL) are presented. Scale bar, $20 \mu \mathrm{m}$.

brane sheets in culture, which resemble compacted myelin membrane in vivo (Aggarwal et al., 2011b). Using this model system, our aim was to establish a new assay where the interaction between two myelin bilayers can be reconstituted. Therefore, we investigated the interaction between prepared myelin particles and primary oligodendrocytes (Figure 3.14).

\subsubsection{The preparation and characterization of myelin particles}

Myelin membrane, purified from adult mice brain, was sonicated to obtain myelin particles. To establish the binding assay those particles were required that resemble the compacted area of myelin (enriched in PLP) and have low content of glycoproteins, which localize to noncompact region. For this purpose, we used Concanavalin A beads to remove glycoprotein fraction from prepared particles. The obtained fractions were then treated with calcium to eliminate MBP, which exerts strong adhesion forces on negative surfaces. The purified particles were then analyzed using western blotting. A visible reduction in MAG and CNPase level was observed, during preparation. Furthermore, the amount of 
Figure 3.15: Preparation and characterization of myelin particles. Purified myelin membrane was sonicated and subjected to ConA glycoprotein isolation beads. The flow through (FT) was then incubated with calcium for $3 \mathrm{hr}$ at $37^{\circ} \mathrm{C}$. Western blot analysis was performed for different fractions for the indicated proteins.

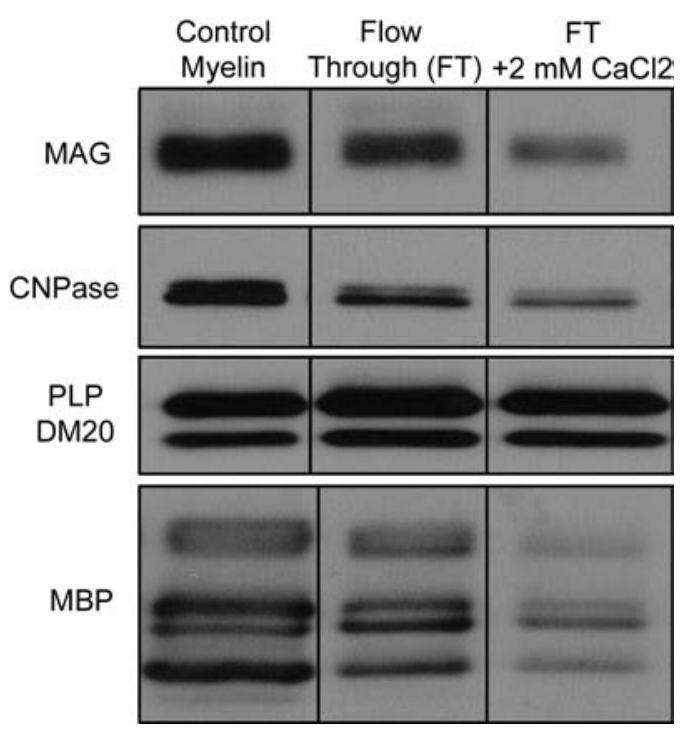

MBP was also decreased whereas PLP level was unchanged (Figure 3.15).

Myelin particle preparation was also explored using immunostaining. We used antibodies which react against PLP and CNPase and stained the particles with the lipophilic dye PKH26 as the reference. After preparation most particles stained for PLP whereas the staining for CNPase was reduced, confirming the enrichment of compact myelin protein in these particles (Figure 3.16).

\subsubsection{Myelin particles preferentially bind to oligodendrocyte membrane surface}

We evaluated the binding of prepared myelin particles to different glial cells in the CNS. We found that these particles bind preferentially to mature oligodendrocytes in comparison to astrocytes and A2B5-positive cells, immature oligodendrocytes. Meanwhile the particles were internalized by the phagocytic microglial cells and subsequently accumulated in their cytoplasmic compartments (Figure 3.17).

Z-stack confocal imaging revealed that the myelin particles were localized on the surface of oligodendrocytes, thus excluding possible engulfment by these cells (Figure 3.18). 


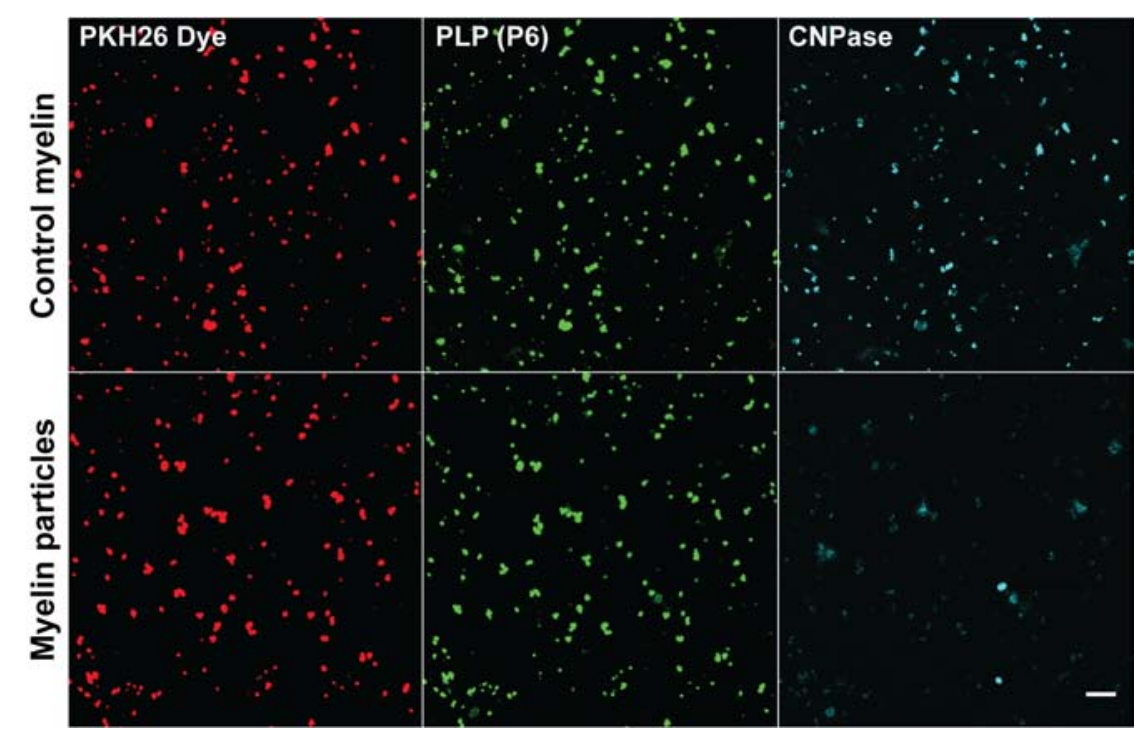

Figure 3.16: Immunolabeling of myelin particles. Control myelin and purified particles were immunolabeled with antibodies against PLP and CNPase and co-stained with the lipophilic dye PKH26 for the reference. Scale bar, $20 \mu \mathrm{m}$.

\subsubsection{PLP plays a role in the interaction of myelin particles with OLs}

PLP and MBP are the main components of CNS myelin proteins (Jahn et al., 2009). While MBP has been shown to form a molecular sieve and mediate close apposition of myelin bilyers on the cytsolic side (Aggarwal et al., 2011b), the mechansims which regulate adhesion of myelin membranes on the extracelullar leaflet remain obscure. We explored the role of PLP in mediating myelin membrane adhesion and compaction. For this, we used myelin particles and oligodendrocytes from PLP knockout (KPLP) mice (Klugmann et al., 1997). We incubated either wild type or KPLP myelin particles with wild-type (WT) OLs and measured the number of bound particles per unit cell area. The number of particles bound per cell was significantly higher in WT cells compared to the KPLP cells (Figure 3.19 A, B). We also added WT particles to a mixed culture of WT and KPLP OLs. We again, detected more binding of the particles to WT than KPLP cells (Figure 3.19 C). These data show possible role of PLP in mediating interaction of myelin particles with oligodendrocytes.

Next, we investigated the interaction between myelin particles and OLs when PLP was present only in one of the membranes. Incubation of KPLP cells with either WT or KPLP 
particles revealed a significantly higher interaction for the WT particles (Figure 3.20 A, B). Afterwards, we added KPLP myelin particles to the mixed cultures of WT and KPLP cells. Again, the interaction was higher between WT cells and KPLP particles (Figure 3.20 C). These data show that presence of PLP on one side increases the interaction between myelin particles and oligodendrocytes, suggesting the possible interaction of PLP with other unknown molecules. However, PLP might also interact with another PLP molecule on the opposite membrane.

To obtain further evidence for the adhesive role of PLP, we performed an in vitro myelinmyelin interaction assay. First, we stained purified myelin particles with two different dyes, PKH26 (red) and PKH67 (green). Second, we incubated these two different population of particles on a rotating device for $1 \mathrm{hr}$. After incubation, we added the particles onto coverslips followed by fixation. Next, we analyzed the percentage of particles which interact amongst total population. Using this system we studied the interaction between WT-WT and KPLP-KPLP myelin particles. In comparison to KPLP myelin, higher interaction was observed between WT particles (Figure 3.21) supporting the adhesive role of PLP.

Reconstitution of transmembrane proteins in artificial vesicles is a useful tool to study their function (Ter Beest et al., 1994; Bizzozero and Howard, 2002). To obtain direct evidence of PLP function in myelin membrane adhesion, we prepared liposomes containing PLP. Because of its hydrophobicity, PLP can be recovered within the lipid fraction during myelin lipid extraction. Taking advantage of this property, liposomes were prepared from myelin extracted lipids and their aggregation was monitored (Figure $3.22 \mathrm{~A}$ ). We observed more aggregation between WT myelin-derived vesicles in comparison to ones derived from KPLP myelin lipids (Figure $3.22 \mathrm{~B}$ ). These absorbance assays were complimented by negative staining electron microscopy which also revealed greater accumulation for WT vesicles (Figure $3.22 \mathrm{C}$ ). Altogether, these findings suggest an adhesive role for PLP in myelin bilyer interaction on the extracellular sides. 


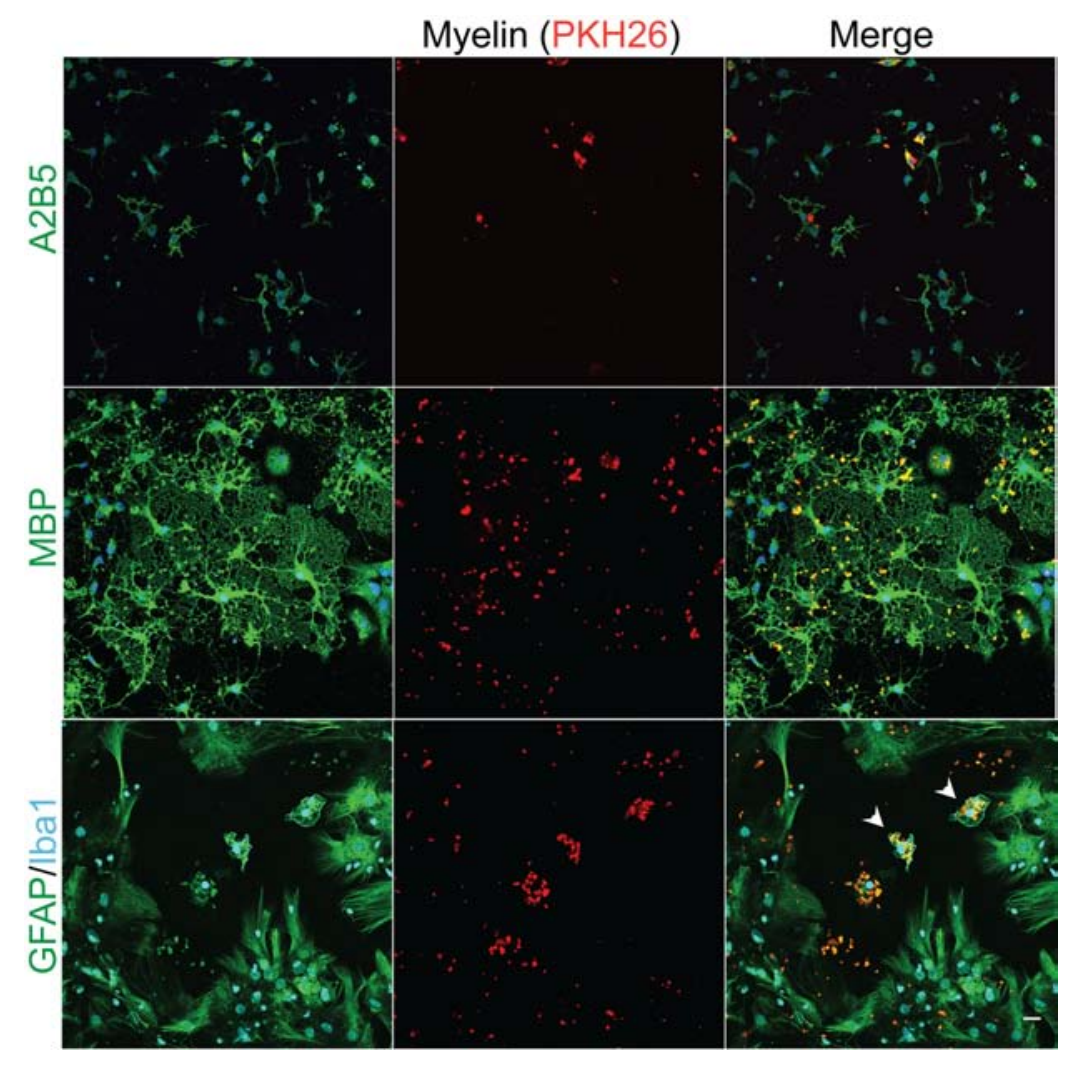

Figure 3.17: Interaction of myelin particles with different glial cells. Purified labeled particles were added to different glial cell preparations including; astrocytes, microglia, undifferentiated and differentiated oligodendrocytes. MBP-positive cells showed high affinity for myelin particles whereas A2B5-positive cells had no interaction with these particles. Moreover, astrocytes revealed low binding for the particles which were in contrast endocytosed by microglia (arrowheads). Scale bar, $20 \mu \mathrm{m}$.

\subsubsection{PLP increases physical stability of myelin}

If PLP plays a role in myelin compaction it is likely that this protein influences myelin stability (Klugmann et al., 1997). To test this possibility, we investigated the physical stability of myelin in the absence of PLP. We examined the morphological differences between myelin derived from WT and PLP knockout mice. Therefore, the size distribution of prepared myelin particles from two different samples were analyzed. When compared to KPLP myelin, WT particles showed a distribution tendency towards larger size (Figure 3.23), indicating a stronger physical stability of particles in the presence of PLP during myelin preparation. Whether the cis interaction between PLP molecules in the same lipid 


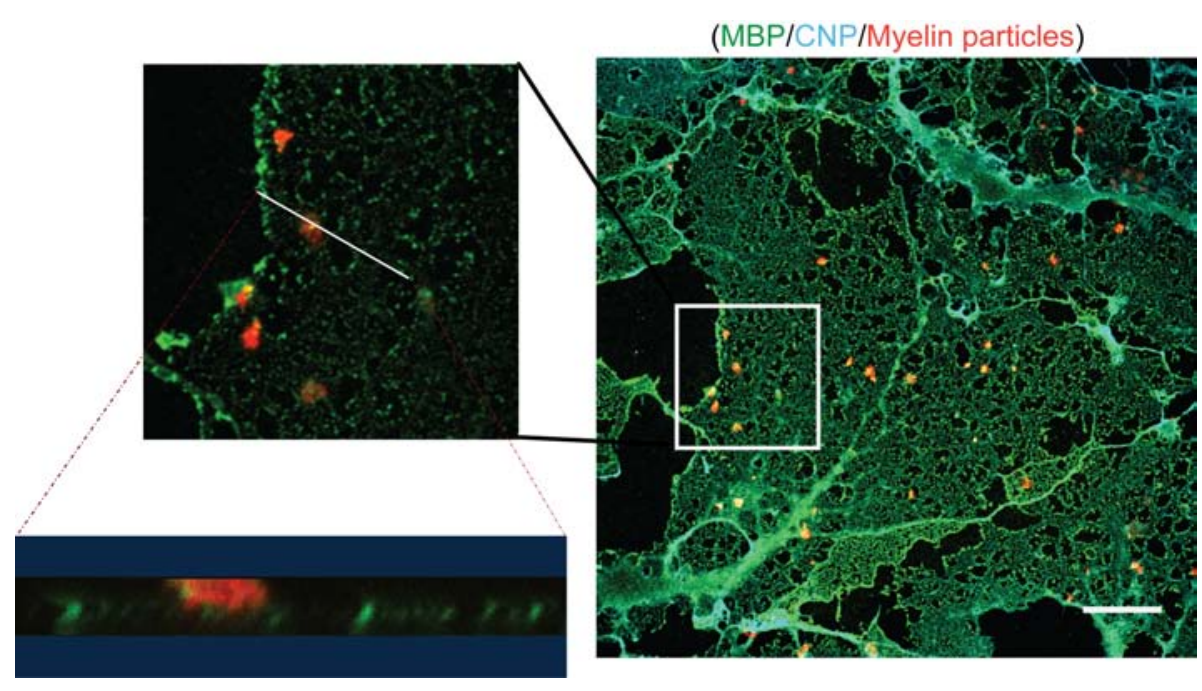

Figure 3.18: Myelin particles interact with the myelin-like membrane surface. Myelin particles were added to 5-day-old oligodendrocytes and analyzed for the position of bound particles using confocal microscopy. ImageJ analysis revealed the localization of myelin particles with the surface of oligodendrocyte myelin-like membrane. Scale bar, $20 \mu \mathrm{m}$.

bilayer or their trans association from the two opposite membranes increases myelin stability, cannot be concluded from this observation.

To support the role of PLP in myelin membrane stability, the interaction of myelin particles with oligodendrocytes was investigated using single-particle force spectroscopy. The obtained force-distance curves (Figure 3.24 A) revealed mean maximum adhesion strengths of $248 \pm 93 \mathrm{pN}$ for WT myelin particles with WT oligodendrocytes and $210 \pm 87 \mathrm{pN}$ for KPLP particles interacting with PLP knockout cells. According to the performed Wilcoxon rank-sum test, the distributions showed significant differences $(p<0.05$, Figure $3.24 \mathrm{~B}$ ). Interestingly, force distance curves of measurements with KPLP particles showed significantly higher perturbations than those for measurements with WT particles (Figure $3.24 \mathrm{~A}$ ). We attributed these increased fluctuations during approach and retraction to the instability of KPLP particles leading to thermally excited oscillations of the cantilever due to undulations of the liquid particles. This assumption is also supported by the fact that in case of WT particles $80 \%$ of the recorded force curves could be analyzed, whereas for KPLP particles only $60 \%$ of the curves could be used due to perturbations occurring during the contact regime. Power spectral density obtained by Fast Fourier analysis of the approach curves revealed the frequency-dependent motion of KPLP particles 

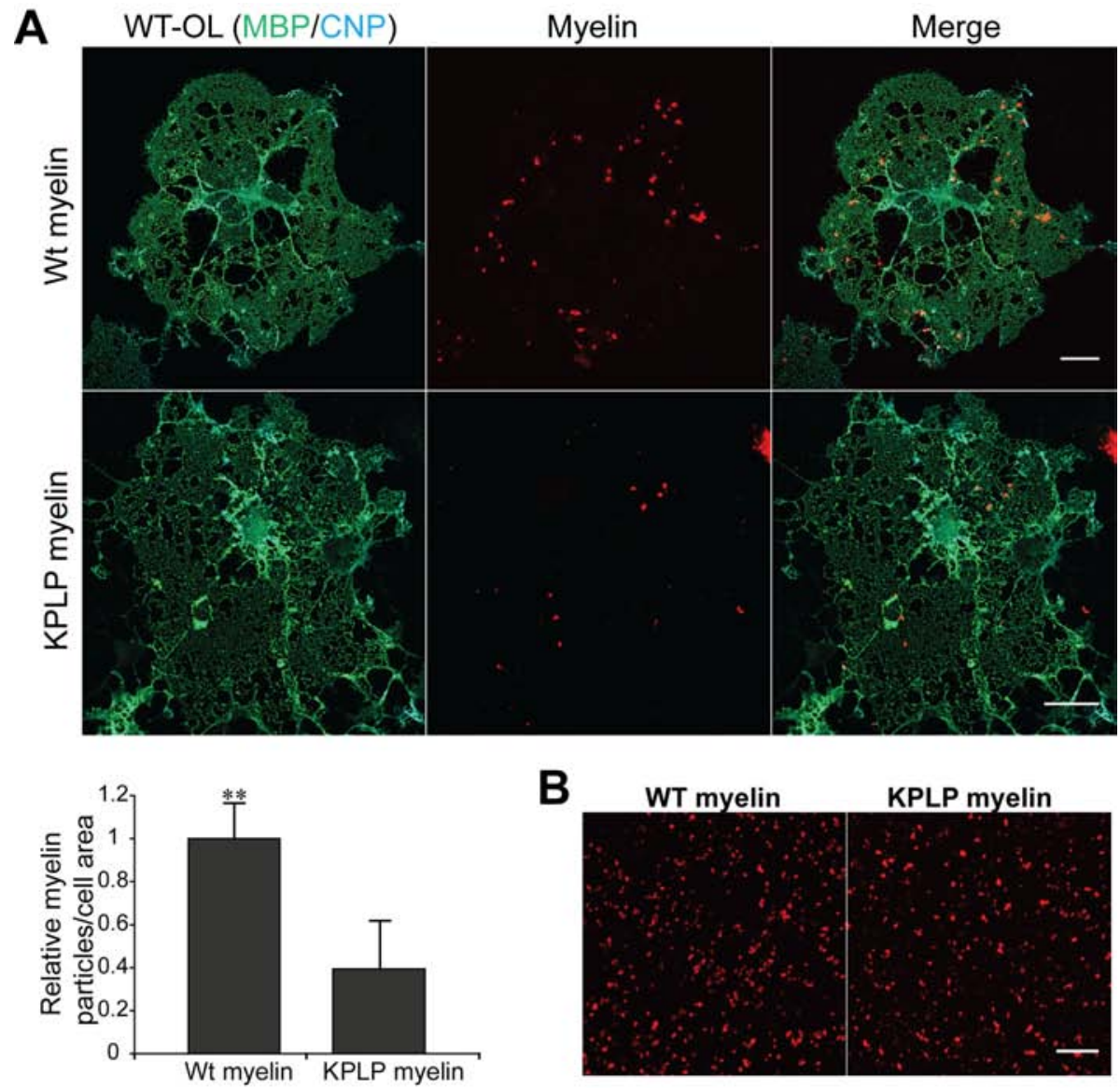

C
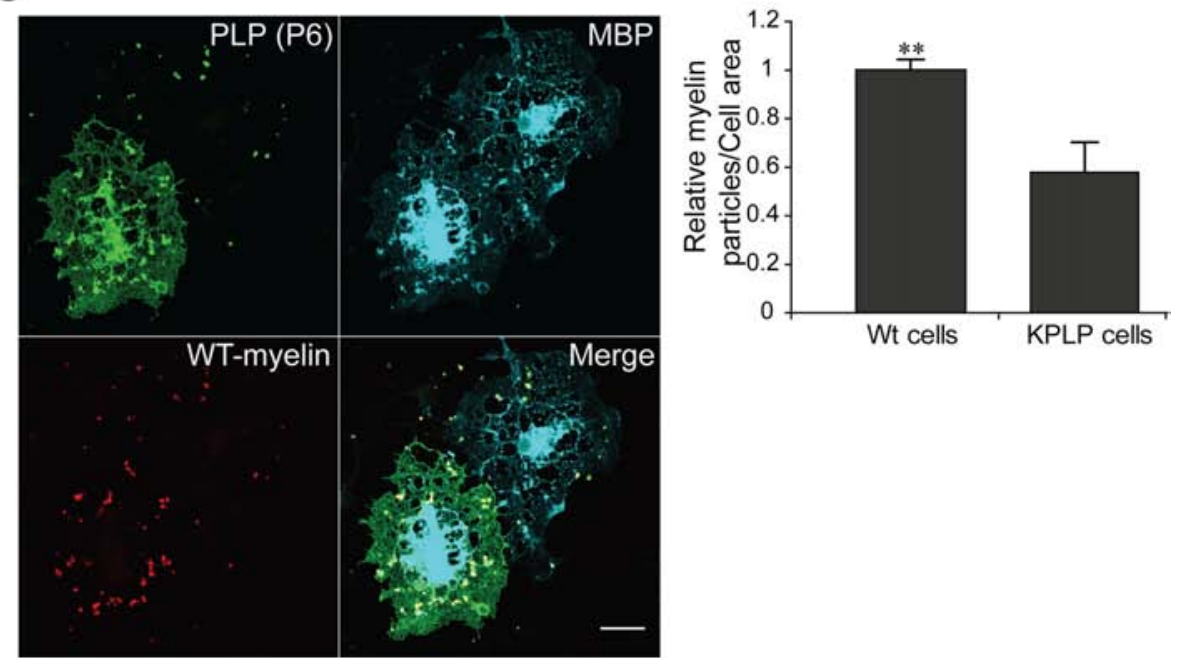

Figure 3.19: PLP increases particle-cell interaction. (A) WT and KPLP myelin particles were added to WT cells and the number of particles per cell area was calculated. WT particles showed more affinity towards OLs compared to KPLP myelin. (B) The reference images for two different particles are represented. (C) WT myelin particles were added to a mixed culture of WT and KPLP cells and the number of particles per cell area was calculated. An increase in binding for WT oligodendrocytes was observed compared to the KPLP cells. ( $n=3,{ }^{* *} p<0.01 ; t$-test). Scale bar, $20 \mu \mathrm{m}$. 

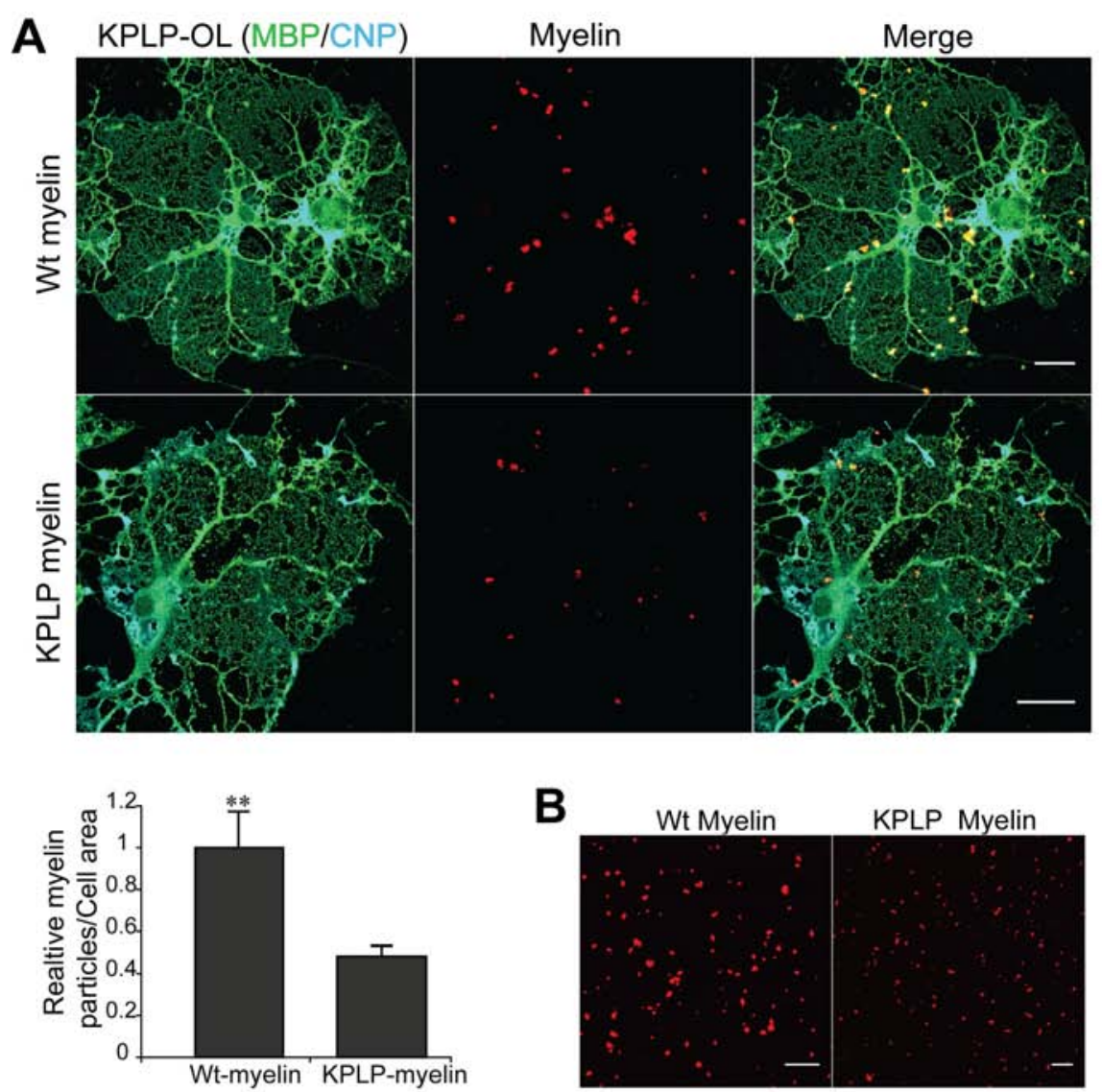

C
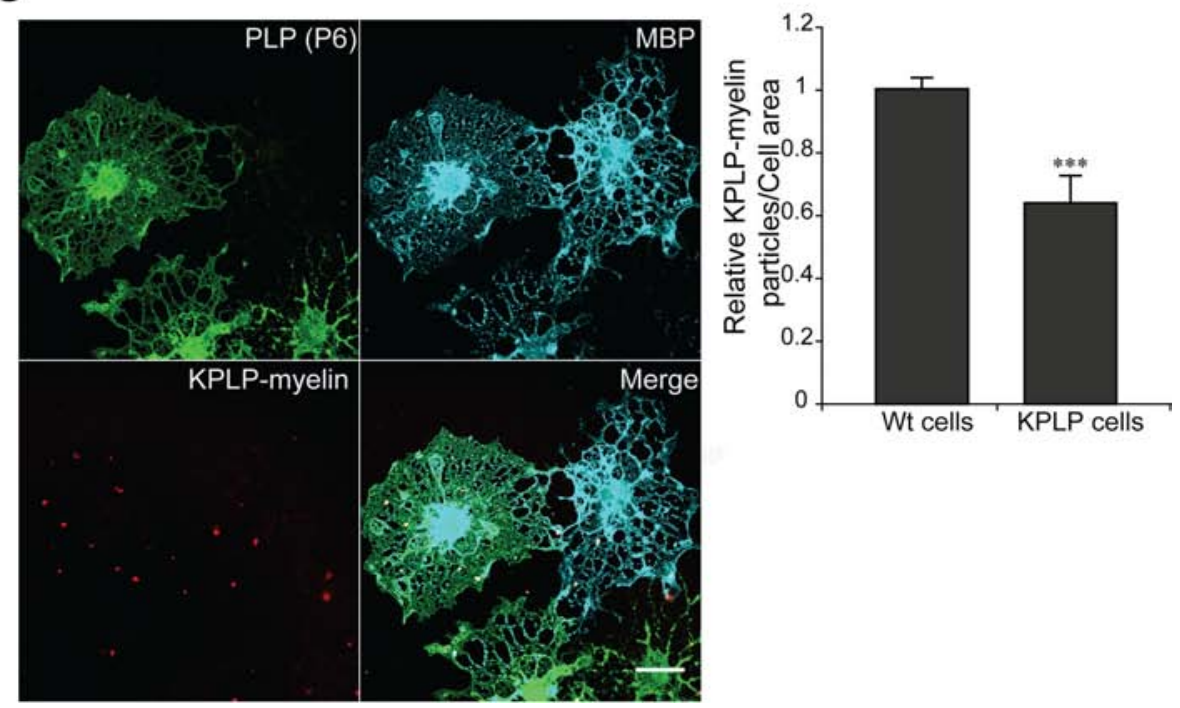

Figure 3.20: Presence of PLP in one side of interacting membranes, increases the binding of particles to oligodendrocytes. (A) WT and KPLP myelin particles were added to KPLP cells and the number of particles per cell area was calculated. WT particles showed more affinity toward OLs compared to KPLP myelin. (B) The reference images for two different particles are represented. (C) KPLP myelin particles were added to a mixed culture of WT and KPLP cells and the amount of particles binding was measured. An increase in binding for WT oligodendrocytes was observed compared to the KPLP cells. ( $n=3,{ }^{* *} p<0.01 ;{ }^{* * *} p<0.001 ; t$-test). Scale bar, $20 \mu \mathrm{m}$. 

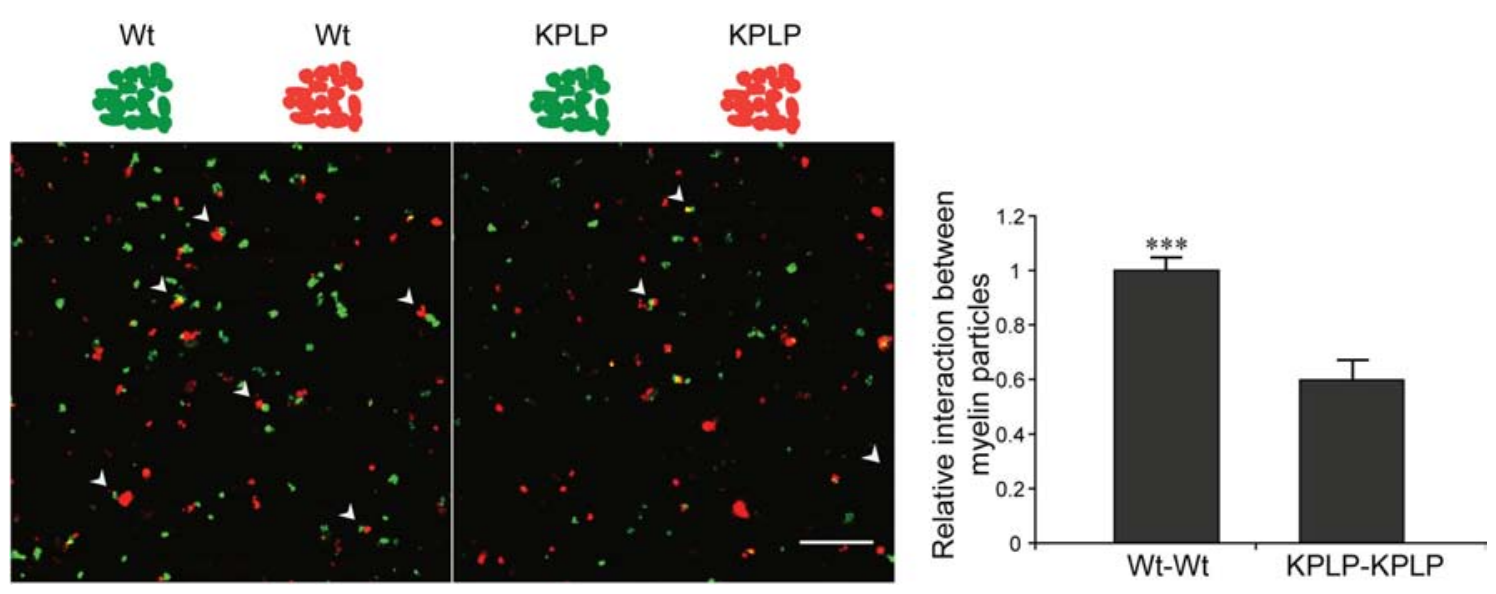

Figure 3.21: PLP increases the interaction between myelin membrane particles. Myelin particles were labeled with two different dyes; PKH26 (red), and PKH67 (green). After incubation for 1 hour at room temperature the mixture of particles was applied on coverslips for $3 \mathrm{hr}$ and then was fixed. The percentage of bound particles (arrowheads) was measured using confocal imaging. Higher interaction between WT particles was observed compared to the KPLP myelin. ( $\mathrm{n}=3,{ }^{* * *} p<0.001 ; t$-test). Scale bar, $20 \mu \mathrm{m}$.

in comparison to wild-type ones (Figure $3.24 \mathrm{C}$ ). Whereas WT particles were stable and thus perturbed cantilever movement in a negligible fashion, the unstable KPLP particles resulted in oscillations during the experiment. This oscillations are reflected in significant peaks within the recorded frequency spectrum. The occurrence of a high number of peaks in the spectrum for KPLP particles stressed the increased flexibility of this type of particle. These data demonstrate that PLP not only plays an essential role in the stabilization of the myelin particles, but is also important for the interaction between myelin and myelinlike membrane.

To investigate the stability of myelin membrane in the absence of PLP in vivo we performed electron microscopic analysis. Electron micrographs of optic nerve prepared by high pressure freezing (HPF) approach did not show any abnormality in area with optimal preservation (Figure $3.25 \mathrm{~A}$ ) which was consistent with previous observations (Klugmann et al., 1997; Möbius et al., 2008). However, area with sub-optimal preservation displayed more lamella splitting for the mutant myelin (Figure $3.25 \mathrm{~A}$ ). This has been explained by fixation artifact occurring during sample preparation (Möbius et al., 2008). Therefore, to obtain reproducible information, we applied conventional embedding fixation. Analysis of 
A

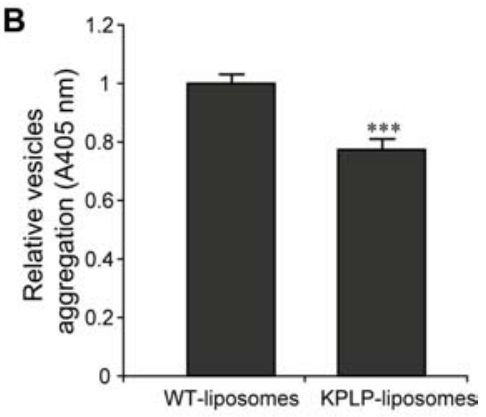

C

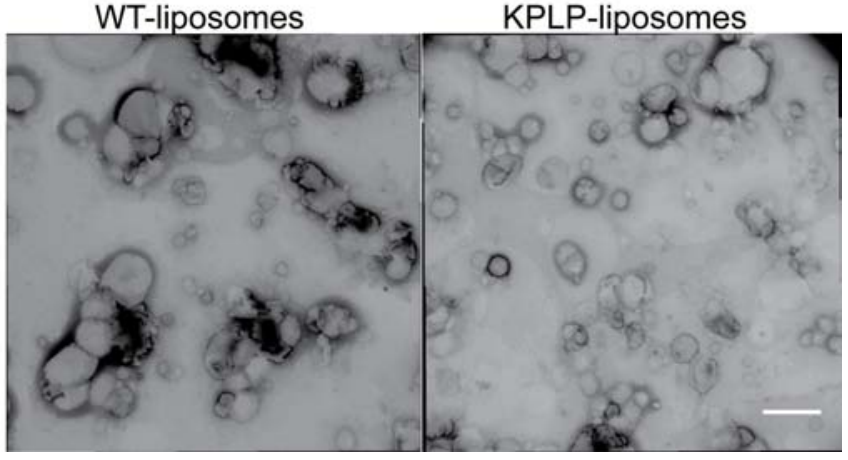

Figure 3.22: PLP increases aggregation of myelin-derived liposomes. (A) Liposomes were prepared by dialysis approach using lipids from WT and KPLP myelin. Western and dot blot analysis were performed to assess PLP and GalC in liposomes, respectively. (B) Liposome aggregation was carried out by measuring the optical density of samples at $405 \mathrm{~nm}$. Liposomes from WT revealed more aggregation in contrast to those from KPLP myelin $\left(n=6,{ }^{* * *} p<0.001 ; t\right.$ test). (C) Typical images of negatively stained WT and KPLP liposomes as visualized by electron microscopy. Scale bar, $2 \mu \mathrm{m}$.

optic nerve from P14 animals revealed a higher periodicity in case of mutant myelin when compared to the WT mice (Figure $3.25 \mathrm{~B}$ ). Taken together, these data provide evidence for the role of PLP in maintaining the stability of myelin membrane in vivo. 

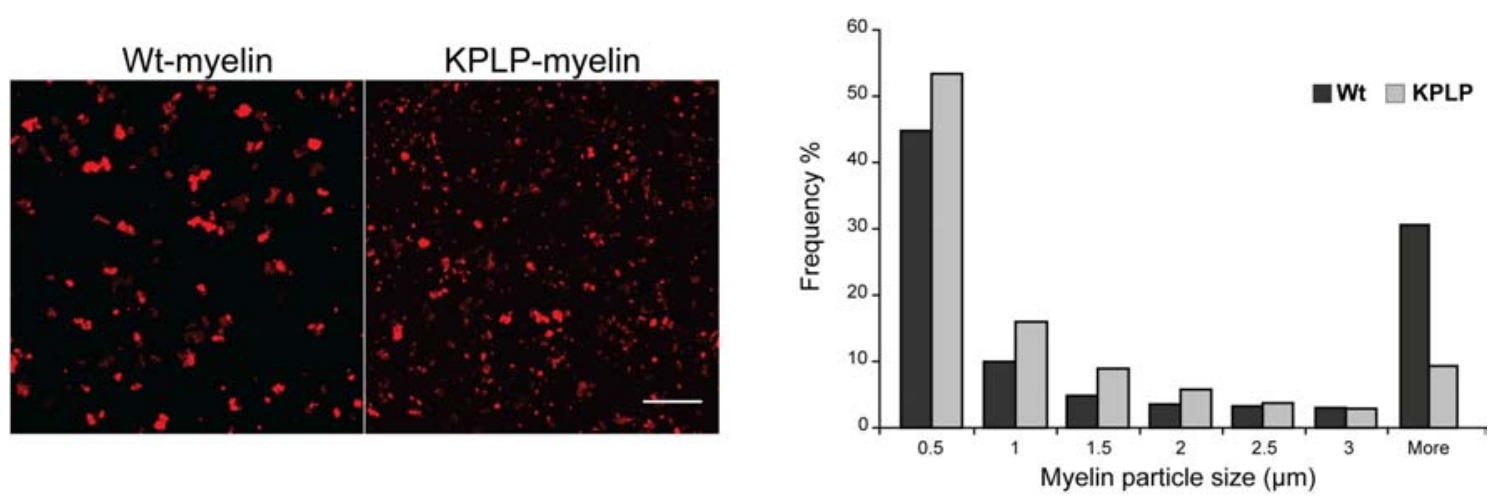

Figure 3.23: PLP increases the size of myelin particles. Purified myelin without sonication was incubated with ConA glycoprotein isolation beads. The passed flow through fractions were stained with PKH26 dye and fixed on coverslips. Using confocal imaging the size distribution of the particles was analyzed. Myelin particles derived from WT mice showed a larger size distribution compared to the KPLP. Scale bar, $20 \mu \mathrm{m}$.

In general, adhesive properties of biological membranes rely on different factors: genetic expression of receptors at the cell surface and generic forces (non-specific forces) which require removal of repulsive structures mainly glycocalyx components from membrane (Sackmann and Bruinsma, 2002). Having shown PLP protein as an adhesive molecule in the CNS myelin, we continued to determine the possible effect of glycocalyx removal, which might be necessary for generating generic forces during myelin compaction.

\subsubsection{Reduction in glycocalyx during oligodendrocytes maturation correlates with an increased in membrane adhesiveness}

Lectins are proteins which are extensively used to study glyco-structures in biological systems (Kitada et al., 2011; Niehaus and Trotter, 1997). To analyze the alteration in OLs glycocalyx during their maturation, we used lectin staining approach. We applied different lectins which recognize specific sugar epitops. For this, we exploited the binding of Con A, PNA (Peanut Agglutinin), and WGA (Wheat Germ Agglutinin) lectins to oligodendroglial cells at day 2 and 5 of their differentiation. Whereas all of these lectins stained OLs at day 2 , they only weakly bound to cells at day 5 (Figure 3.26). In addition, we examined RCA 120, SBA, UEA I, and SNA lectins which did not show any binding to differentiated OLs (data not shown). These data indicate that most types of sugar complexes are reduced in 
A

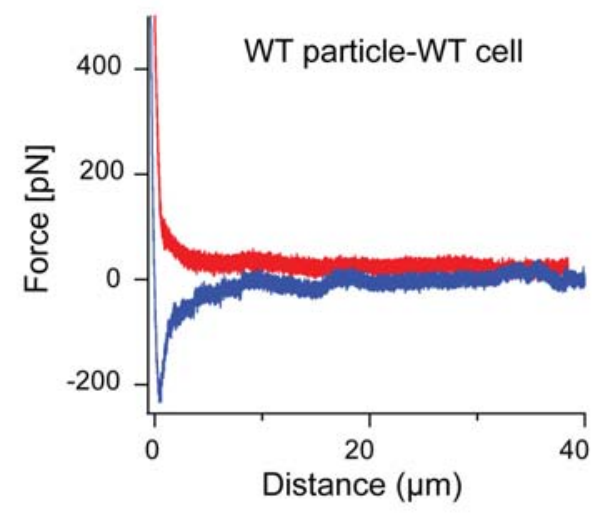

B

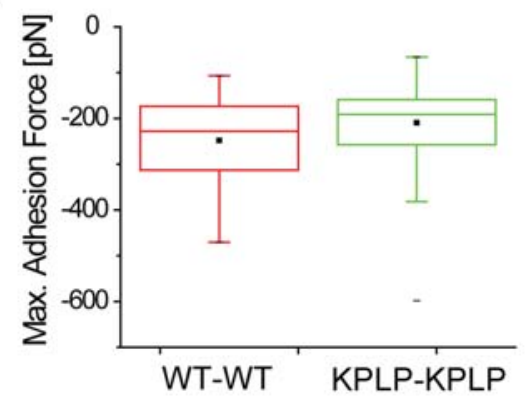

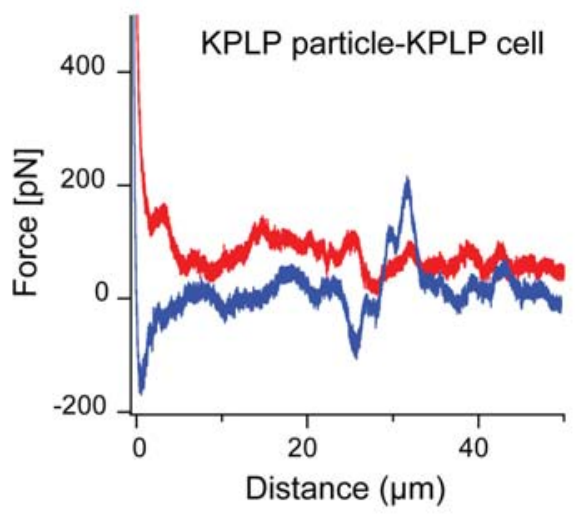

C

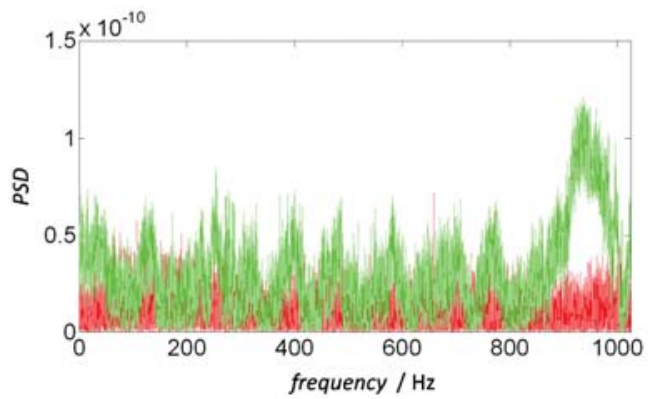

Figure 3.24: Single particle force spectroscopy shows stronger adhesion between particles and cells in the presence of PLP. (A) Exemplary force distance curves of WT myelin particles interacting with WT oligodendrocytes, and KPLP myelin particles interacting with KPLP cells. From these force curves the maximum adhesion force was analyzed; Approach curve (red), Retraction curve (blue). (B) Box-plots for the maximum adhesion force of KPLP myelin particles interacting with KPLP oligodendrocytes (green) and WT myelin particles interacting with WT cells (red), are represented. According to the Wilcoxon rank-sum test, both distributions are significantly different at the 0.05 level $(p=0.006)$. (C) Power spectral density obtained from Fast Fourier analysis of the corresponding base-line corrected approach curves; approach curves of KPLP particle (green) and of WT particle (red) bound to the poly-D-lysin coated cantilever, were recorded with a data rate of $2049 \mathrm{~Hz}$. For analysis only data points before reaching the contact point were used. 

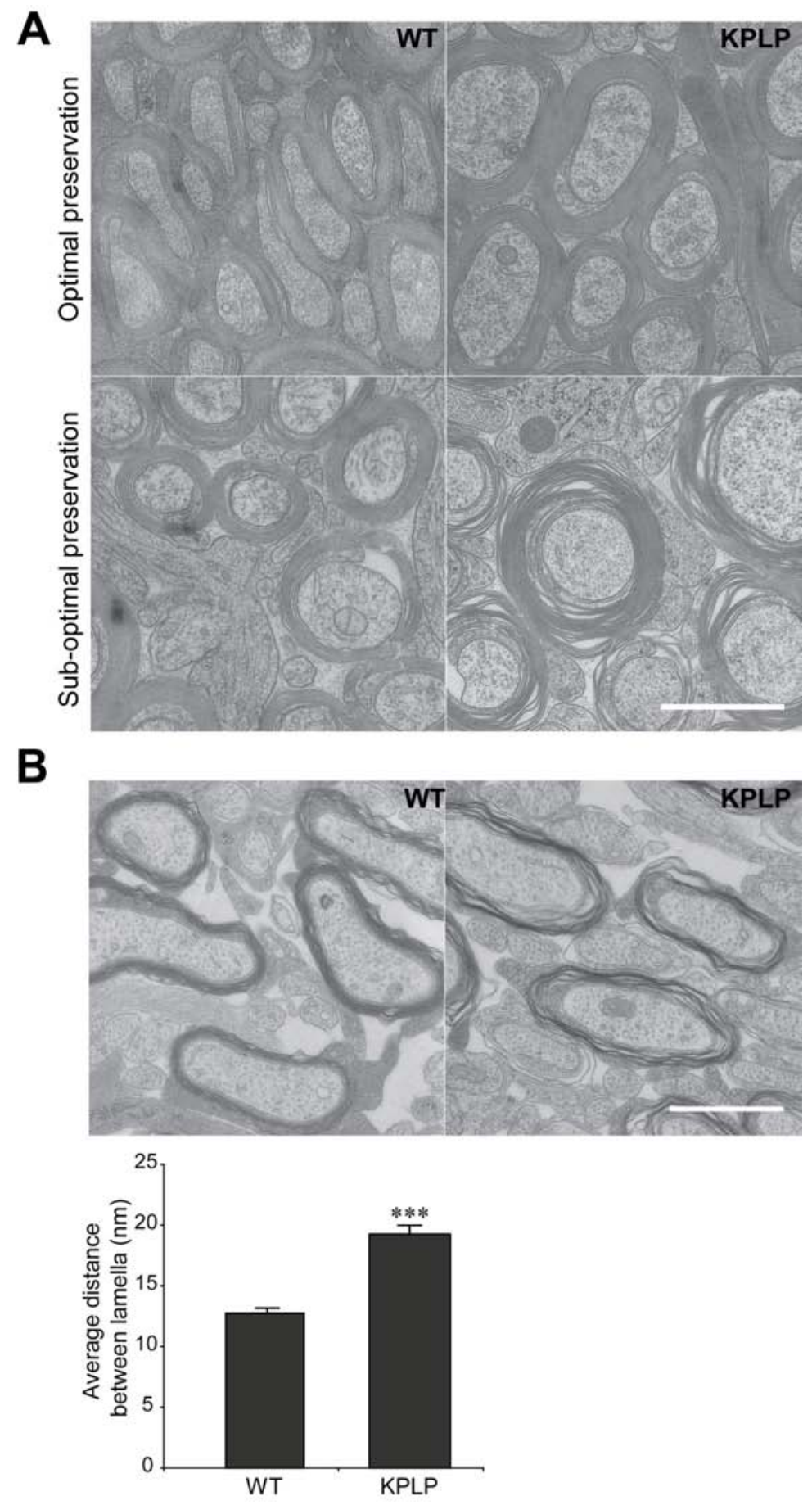

Figure 3.25: The stability of myelin is reduced in the absence of PLP. (A) Optic nerves from P21 mice were prepared by high pressure freezing (HPF) approach. Electron micrographs of the samples revealed normal myelin structure for KPLP in areas with optimal preservation. However, KPLP samples in areas with sub-optimal preservation displayed increased lamella splitting compared to WT. (B) Optic nerves from P14 mice were prepared by conventional embedding fixation. Electron micrographs of the samples were obtained and the average distance between myelin lamella was measured. ( $\mathrm{n}=15$ images from 3 animals, ${ }^{* * *} p<0.001 ; t$-test). Scale bar, $1 \mu \mathrm{m}$. 
the surface of OLs during their maturation.

Based on lectin staining, we hypothesized that the alteration in glycocalyx may change OLs membrane adhesion during their maturation. To investigate this possibility, we performed a binding assay, in which myelin particles were added to OLs at day 3 and 5 of their differentiation, and the number of particles per cell area was calculated. An obvious increase in particles binding was detected for the cells at day 5 compared to those at day 3 (Figure $3.27 \mathrm{~A}$ ). Since PLP was shown to mediate the particle-OL interaction and the level of this protein is increased during OLs maturation, we performed the same experiment using PLP knockout cells. An increase in particles binding during maturation of KPLP cells was also observed (Figure $3.27 \mathrm{~B}$ ). Taken together these data indicate that another factor beside PLP protein is involved in increasing myelin adhesiveness during OLs maturation which might be relevant to the reduction in their surface glycocalyx.
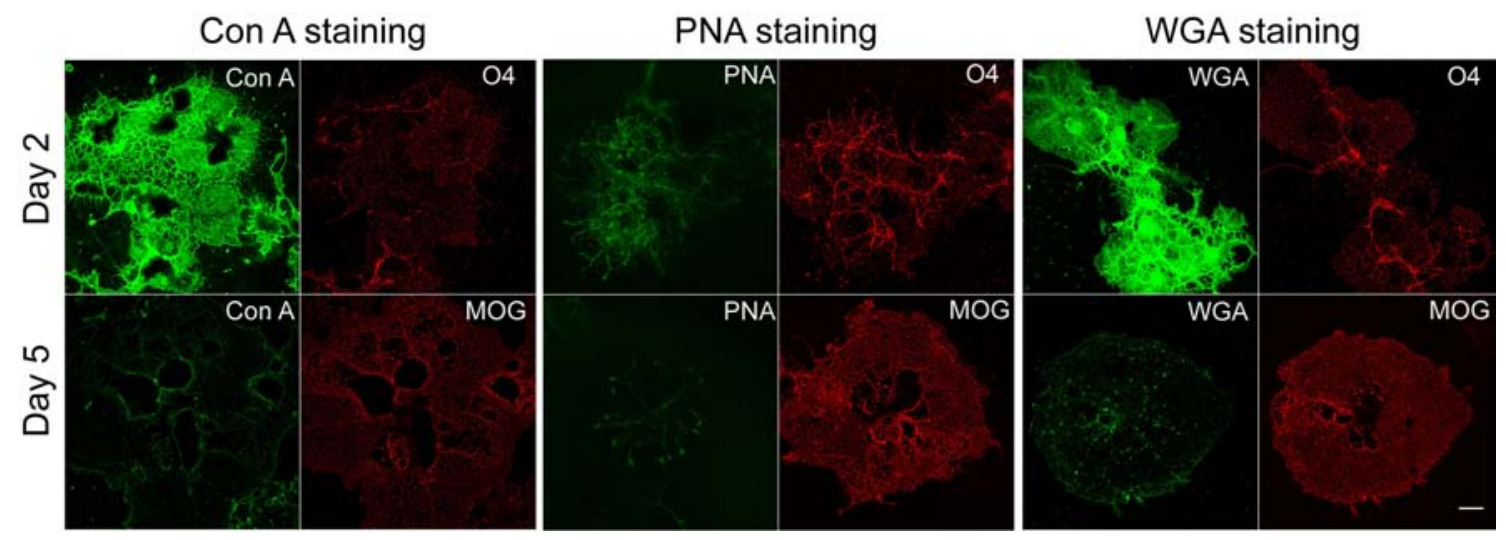

Figure 3.26: A massive reduction in the surface glycocalyx occurs during oligodendrocytes maturation. Cultured oligodendrocytes at day 2 and 5 were assessed for their surface glycocalyx using different lectins. ConA, PNA, and WGA staining showed a clear reduction in the cell surface sugars upon maturation of the cells. Scale bar, $20 \mu \mathrm{m}$. 

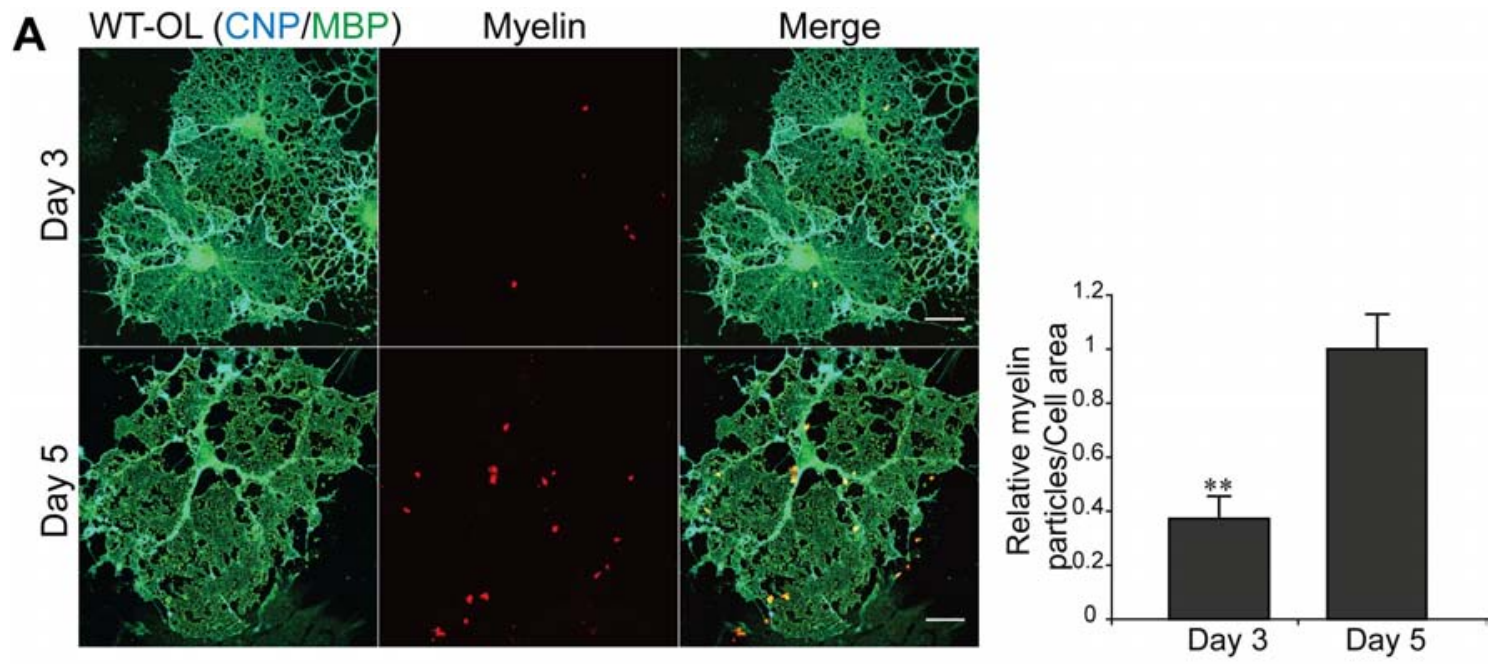

$\mathrm{B}$
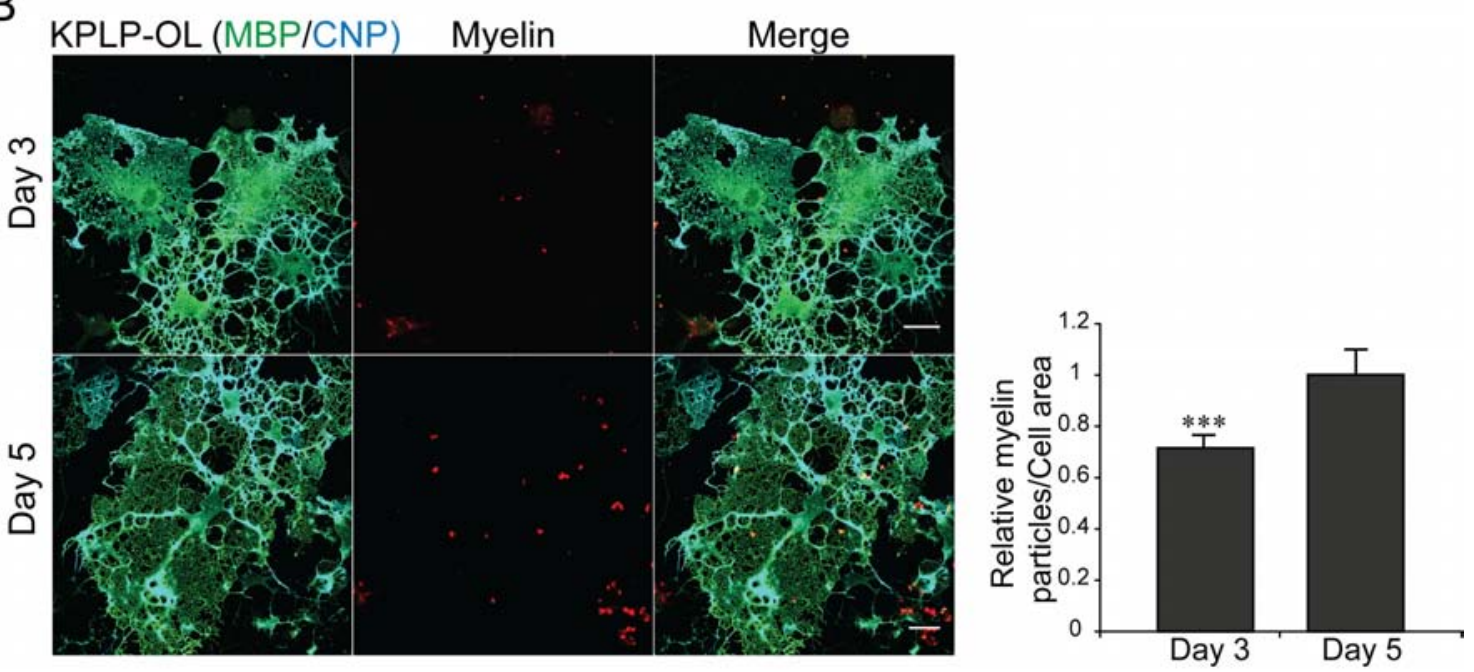

Figure 3.27: Myelin-like membrane adhesiveness is increased during oligodendrocytes maturation. (A) Myelin particles were added to cells at different days in development and the number of particles per cell area was measured. Particle binding was higher in immature (day 3) compared to mature (day 5) cells. ( $n=3,{ }^{* *} p<0.01 ; t$-test) (B) Myelin particles were incubated with KPLP cells in different time points of their differentiation. An increase in particle binding was detected for cells during later steps of maturation $\left(n=4,{ }^{* * *} p<0.001 ; t\right.$-test). Scale bar, $20 \mu \mathrm{m}$. 


\subsubsection{Reduction of sialic acid from myelin-like membrane increases OLs affinity towards myelin particles}

Due to its negative charge, sialic acid is an important repulsive molecule which influences cell-cell interaction. For instance, addition of polysialic acids to NCAM reduces the adhesion between two biological membranes (Rutishauser et al., 1990). We, therefore, focused on the role of this molecule during myelin membrane adhesion and compaction. Although WGA binds to sialic acid containing moieties, it also binds to other sugar molecules. To detect sialic acid more specifically, we used MAL II lectin, which interacts only with the epitopes containing sialic acid. OLs at day 2 and 5 as well as microglial and astrocytic cells were stained for MAL II. Whereas day 2 oligodendrocytes, microglia, and astrocytes were robustly stained with MAL II, OLs at day 5 showed only weak staining (Figure 3.28 A), pointing towards a process in which sialic acid levels go down during myelin membrane maturation.

To obtain further evidence for the reduced levels of sialic acid during myelin membrane maturation, we measured incorporation of this sugar, using metabolic labeling approach. In this method, cells are incubated with an azide-modified molecule which is metabolically incorporated into cells. Using chemoselective ligation or click reaction between the azide and a fluorescent-attached alkyne, the labeled metabolic molecule can be identified in the cells. We used tetraacetylated N-Azidoacetyl-D-Mannosamine (Ac4ManNAz) molecule which is metabolically incorporated into sialic acid biosynthetic pathway. We performed a time course experiment, in which cells at day 0, 2, and 4 were incubated with Ac4ManNAz and the amount of integrated sialic acid was detected after $24 \mathrm{hr}$. We found a significant reduction in sialic acid biosynthesis during oligodendrocytes maturation (Figure 3.28 B). However, time course experiment with astrocytes did not show any differences in sialic acid metabolism (Figure $3.28 \mathrm{C}$ ).

After observing the reduction of sialic acid during oligodendrocyte development in vitro, we investigated whether the level of this sugar in myelinated area is different from other brain regions. Brain slices from adult mice were stained with fluorophore conjugated WGA and MAL II. The staining was far less pronounced in myelin enriched areas, such as corpus callosum, in comparison to other regions of the braine (Figure 3.29). These data suggest that the glycocalyx components are indeed present in low proportion in the myelinated 
A

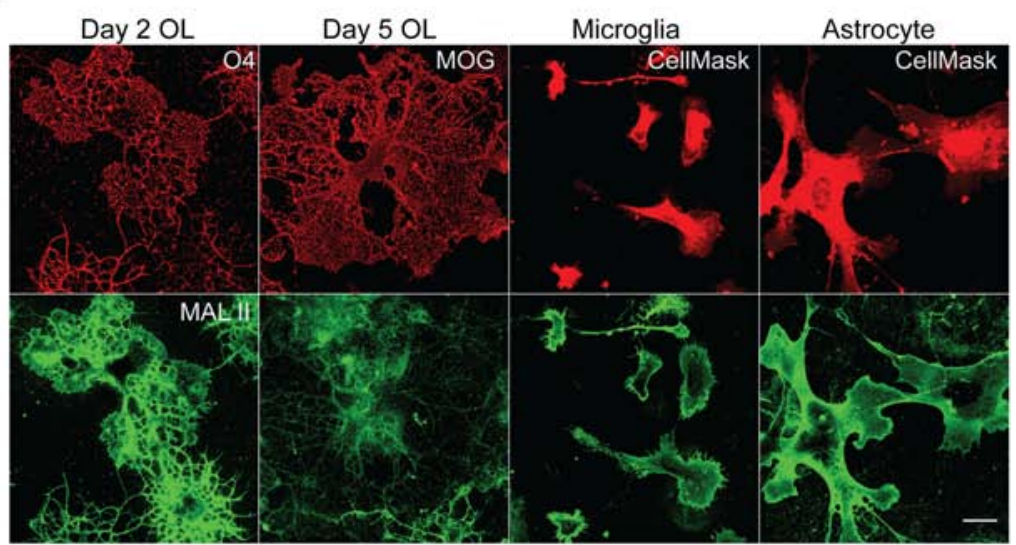

B
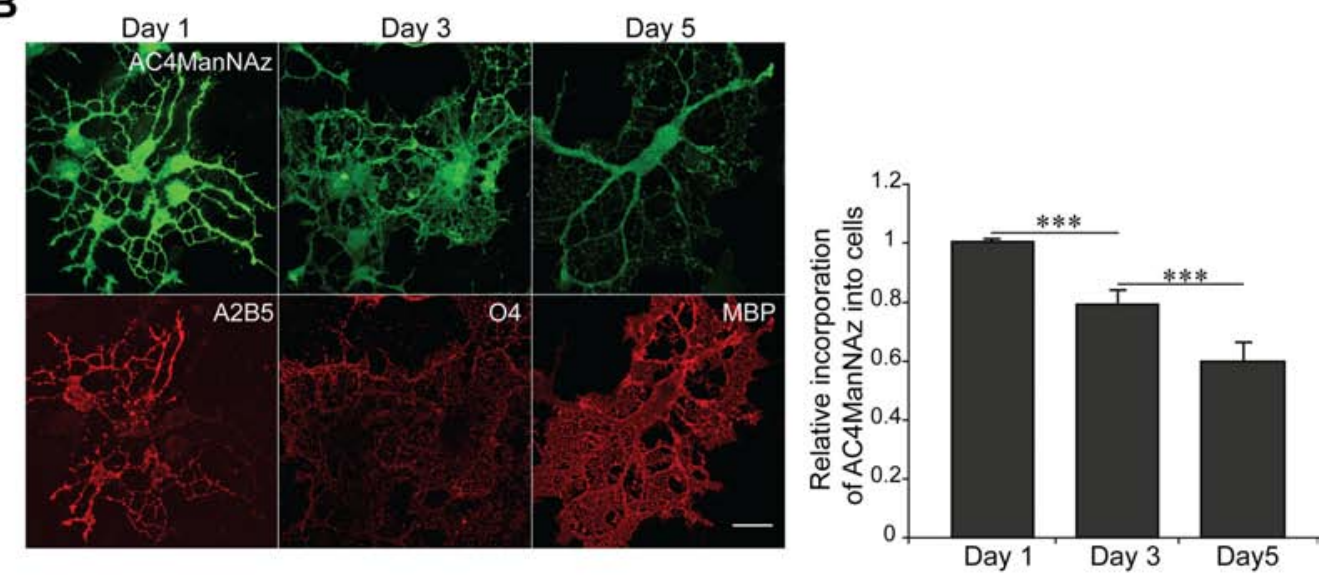

C
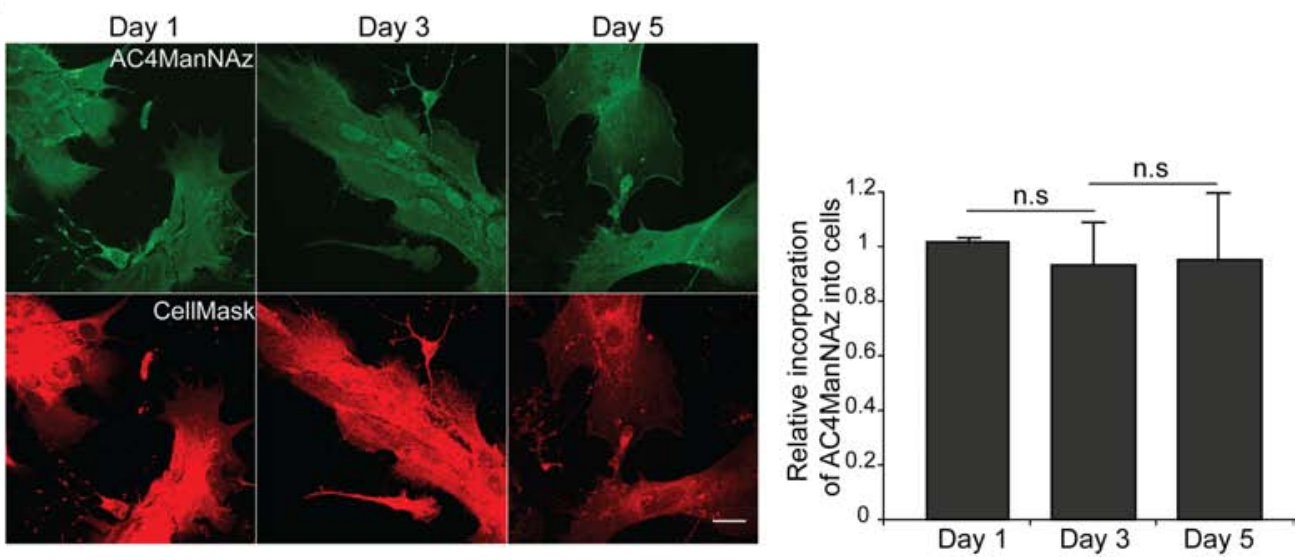

Figure 3.28: Sialic acid content is reduced during oligodendrocytes maturation. (A) Oligodendrocytes at day 2 and 5 after plating as well as microglia and astrocytes, were labeled using MAL II lectin which specifically binds to sialic acid. Confocal images obtained with the same gain demonstrated a reduction in surface sialic acid for oligodendrocytes at day 5 compared to the cells at day 2 and other types of glia. (B) Oligodendrocytes at different stages of their differentiation were treated with $50 \mu \mathrm{M}$ Ac4ManNAz for $24 \mathrm{hr}$. The cells were then labeled using a fluorescent alkyne to detect the incorporated sialic acid. A significant reduction in sialic acid biosynthesis was observed during oligodendrocyte maturation. ( $\mathrm{n}=4,{ }^{* * *} p<0.001$; one-way ANOVA). (C) Astrocytic cells in the similar days were also treated with Ac4ManNAz. However no significant changes were found in sialic acid biosynthesis for these cells. $(n=4, n . s=$ not significant; oneway ANOVA). Scale bar, $20 \mu \mathrm{m}$. 


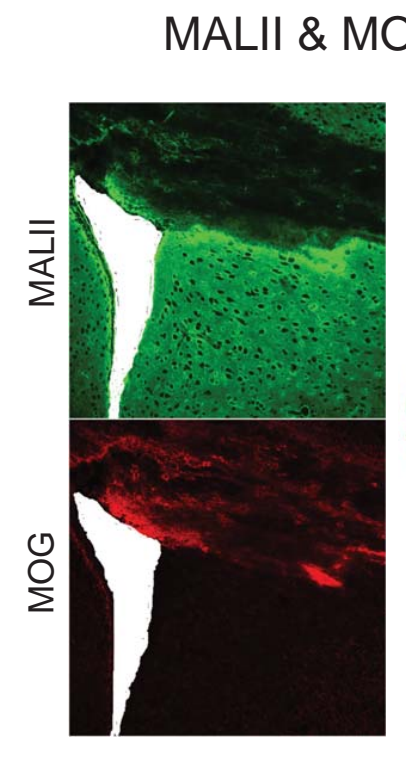

OG staining
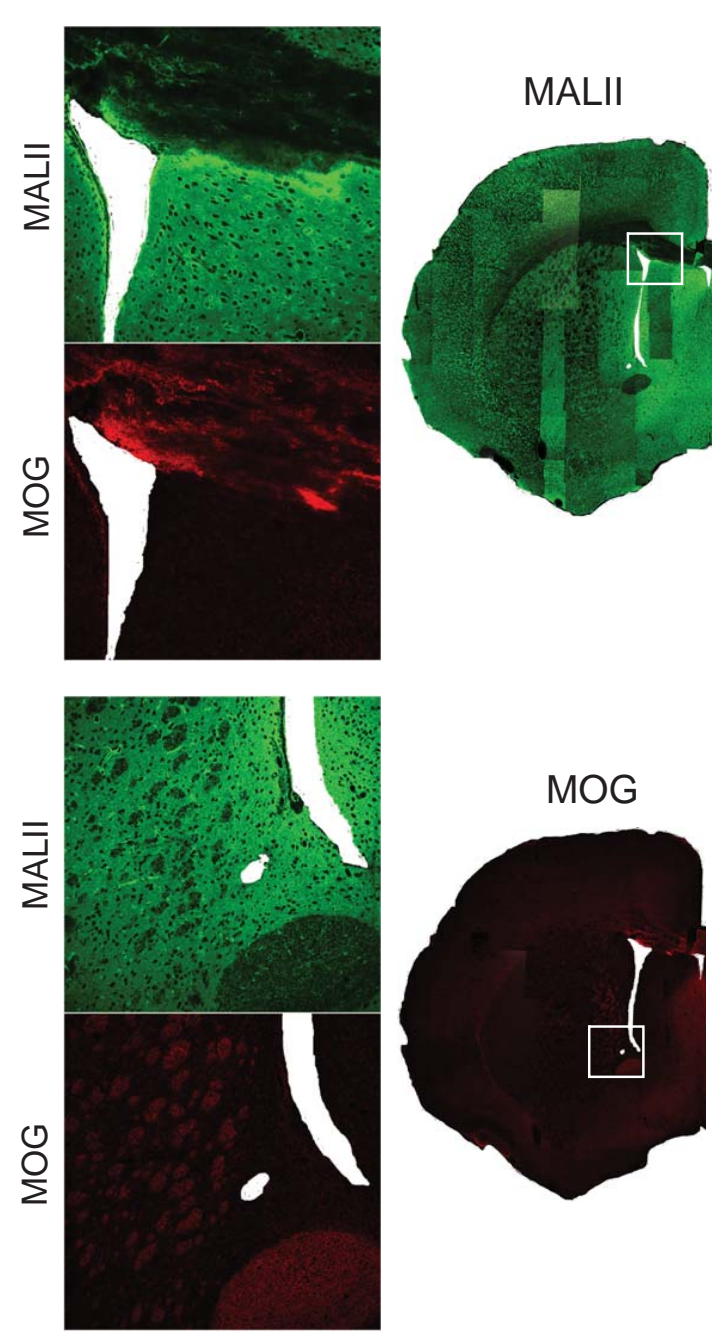

WGA \& MOG staining

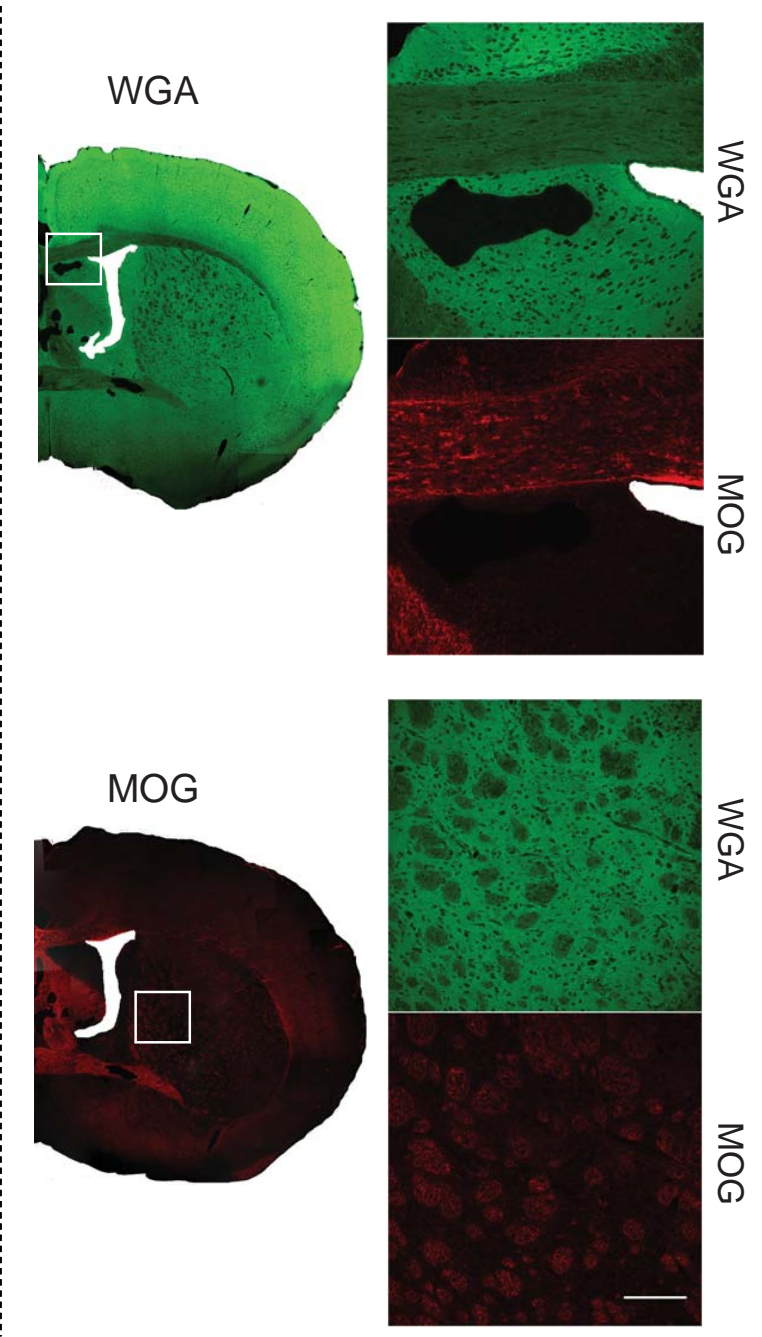

Figure 3.29: Myelinated area possess low sialic acid content. Lectin stainings of brain sections from two-month-old wild-type mice is represented. WGA and MAL II staining showed a striking reduction in lectin binding in area corresponding to myelinated regions, which are shown by staining with antibody directed against MOG. Scale bar, $200 \mu \mathrm{m}$. 
area in vivo.

Having found a reduction in sialic acid level during myelination, we continued to analyze whether removing sialic acid residues from myelin-like membranes can potentially increase their adhesiveness. For this purpose, we used 3-day-old OLs which still have a considerable amount of glycocalyx on their surface. When treated with neuraminidase (sialidase), derived from Clostridium perfringens, cells revealed more interaction with myelin particles (Figure 3.30 A, B). Altogether, we show that during oligodendrocyte maturation, the levels of sialic acid are notably reduced, which in turn correlates with the increased adhesiveness of myelin membrane.

\subsubsection{Negative surface charges interfere with myelin membrane adhesiveness}

Because sialic acid residues contribute to the negative charge of the plasma membrane, we asked whether these charges interfere with the interaction of two opposing membranes. For this, we added a negatively charged molecule, dextran sulfate, to the cells. This substance has been reported to bind to the cell surface (Strilić et al., 2010). Treatment of cells with dextran sulfate significantly reduced the interaction of myelin particles with oligodendrocytes in comparison to the uncharged dextran, which was used as the negative control (Figure $3.31 \mathrm{~A}$ ). We next asked whether neutralization of negative charges could increase myelin-like membrane adhesiveness. To this end, we used cationic protamine sulfate, which has been shown to neutralize the negative charges of sialic acids (Strilić et al., 2010). When added to 3-day-old cells, which still possess glycocalyx as well as negative charges on their surface, protamine sulfate increased the binding of myelin particles to OL surface (Figure $3.31 \mathrm{~B}$ ). These experiments show that cell surface negative charges interfere with the binding of myelin particles to oligodendrocytes. 

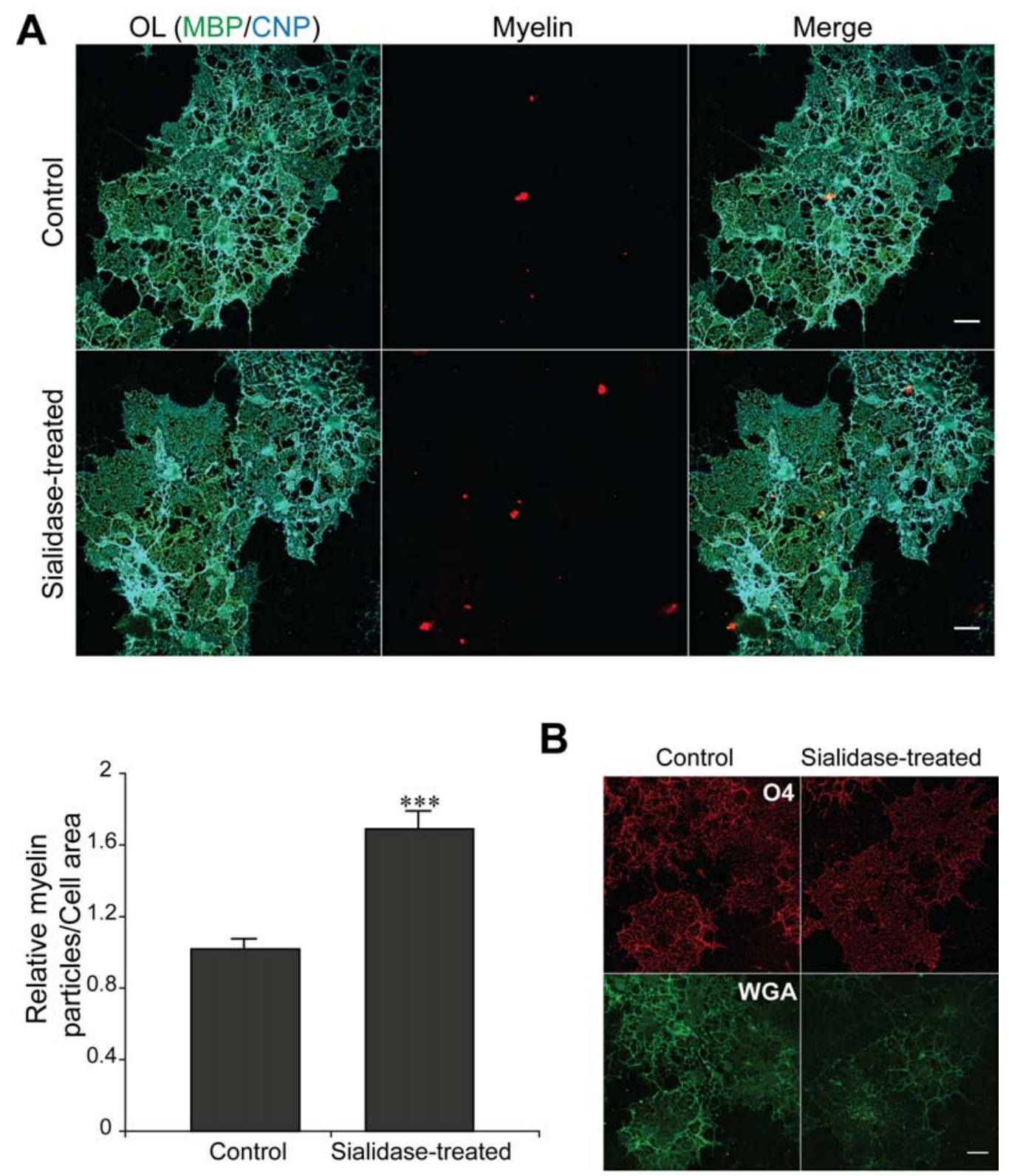

Figure 3.30: Enzymatic removal of sialic acid enhances the interaction between oligodendrocyte surface with myelin particles. (A) Oligodendrocytes at their early stage of differentiation (day 3) were treated with $5 \mathrm{U} / \mathrm{ml}$ sialidase for $1 \mathrm{hr}$ at $37{ }^{\circ} \mathrm{C}$ and the binding assay was performed. Treatment of cells with the enzyme resulted in increased interaction between myelinlike membrane and the particles compared to the control cells. ( $n=4,{ }^{* * *} p<0.001 ; t$-test). (B) The reduction of sialic acid from cell surface after enzymatic treatment was confirmed using WGA lectin staining. Scale bar, $20 \mu \mathrm{m}$. 

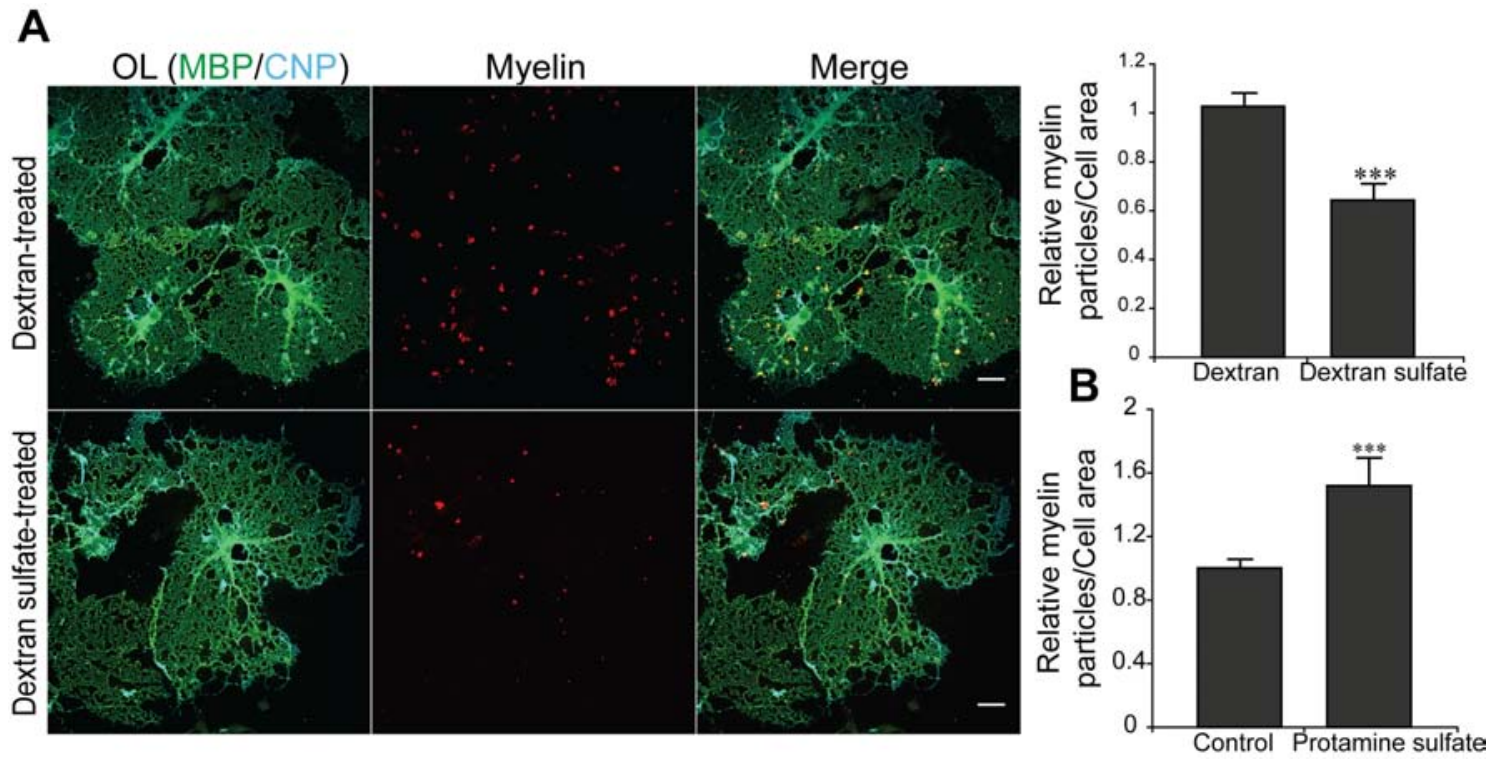

Figure 3.31: Cell surface negative charges reduce myelin particle interaction with oligodendrocytes. (A) Fully differentiated oligodendrocytes were treated with $5 \mathrm{mg} / \mathrm{ml}$ dextran and dextran sulfate for $30 \mathrm{~min}$ at $37{ }^{\circ} \mathrm{C}$. The binding assay was performed and the number of particles per cell area was assessed. The particles binding for cells treated with dextran sulfate was considerably less than those treated with dextran ( $n=4,{ }^{* * *} p<0.001 ; t$-test). (B) Cells in early differentiation step were incubated with $50 \mu \mathrm{g} / \mathrm{ml}$ protamine sulfate for $15 \mathrm{~min}$ at $37{ }^{\circ} \mathrm{C}$. Introducing of myelin particles resulted in more interaction with protamine sulfate-treated cells than controls. ( $\mathrm{n}=5,{ }^{* \star *} p<0.001 ; t$-test). Scale bar, $20 \mu \mathrm{m}$.

\subsubsection{Partially glycocalyx reconstitution reduces particle-cell interaction}

MBP has been reported to form a size barrier which restricts proteins with large cytoplasmic domain to the non-compact areas. By this filtering mechanism, myelin sheets become devoid of most glycoproteins (Aggarwal et al., 2011b). To reconstitute glycoproteins in myelin-like membrane we used OLs from MBP knockout mice (Shiverer). When compared to the WT, shiverer cells showed a significant reduction in myelin particle binding, indicating the interfering of glycoproteins with myelin membranes interaction (Figure $3.32 \mathrm{~A}$ ). To support the inhibitory role of glycoproteins in myelin membrane adhesion, we applied different constructs of S-MAG, which contains many N-linked glycosylation sites. We used a cytoplasmic truncated version of S-MAG (S-MAG N ter) that has been shown to enter the compact area. As a negative control, we used the full length protein (S-MAG), which is restricted to the non-compact region (Aggarwal et al., 2011b). A significant reduction 
in particles binding was observed for cells transfected with truncated MAG in comparison to the control cells that were trasnfected with the full length protein (Figure $3.32 \mathrm{~B}$ ), pointing to the repulsive effect of this glycoprotein on particle-cell interaction.

A

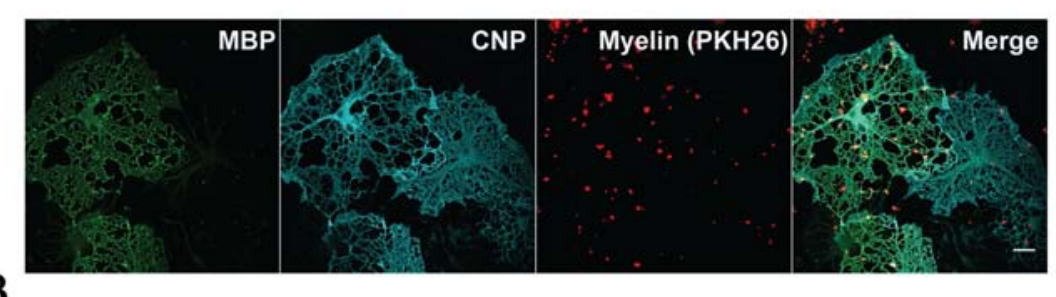

B
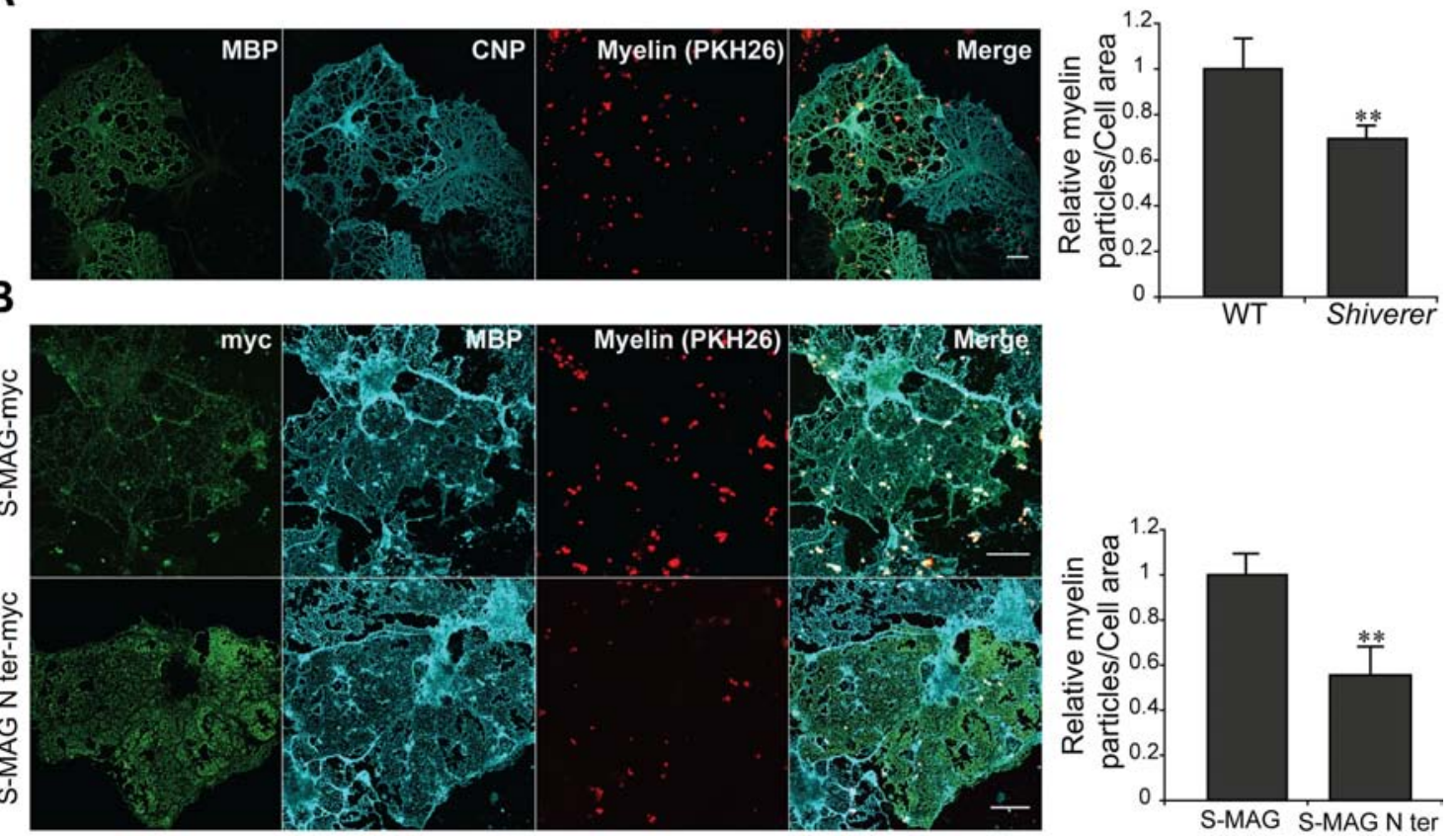

Figure 3.32: Partially reconstitution of glycocalyx into myelin-like membrane decreases its interaction with myelin particles. (A) A mixed culture of WT and Shiverer primary oligodendrocytes was prepared. WT myelin particles were added to the culture and the number of particles per cell area was counted. A considerable decrease in myelin particles binding was detected for Shiverer cells compared to WT. ( $n=5,{ }^{* *} p<0.01 ; t$-test). (B) Oligodendrocytes were transfected with full length (S-MAG-myc), and cytoplasmic truncated (S-MAG N ter-myc) MAG. The cells expressing cytoplasmic-truncated MAG revealed less interaction with myelin particles in comparison to oligodendrocytes transfected with full length protein. $\left(n=4,{ }^{* *} p<0.01 ; t\right.$-test). Scale bar, $20 \mu \mathrm{m}$.

To obtain additional evidence regarding inhibitory role of glyco-structures in myelin membranes interaction, we reconstituted gangliosides, which contain a considerable amount of sialic acid, into OLs membrane. The levels of these lipids have been reported to be reduced during myelin maturation (Cochran et al., 1982). When total brain gangliosides were loaded into OLs, a notable decrease in particles binding was revealed in contrast to the control (Figure $3.33 \mathrm{~A}$ ). Consistent with this observation, introducing of gangliosides into 
myelin liposomes resulted in a dramatic reduction in their aggregation. However, adding sphingomyelin to myelin lipids did not affect liposomes aggregation (Figure $3.33 \mathrm{~B}$ ). Altogether, these data demonstrate a repulsive effect of negatively charged glycoproteins and glycolipids in myelin layers interaction.
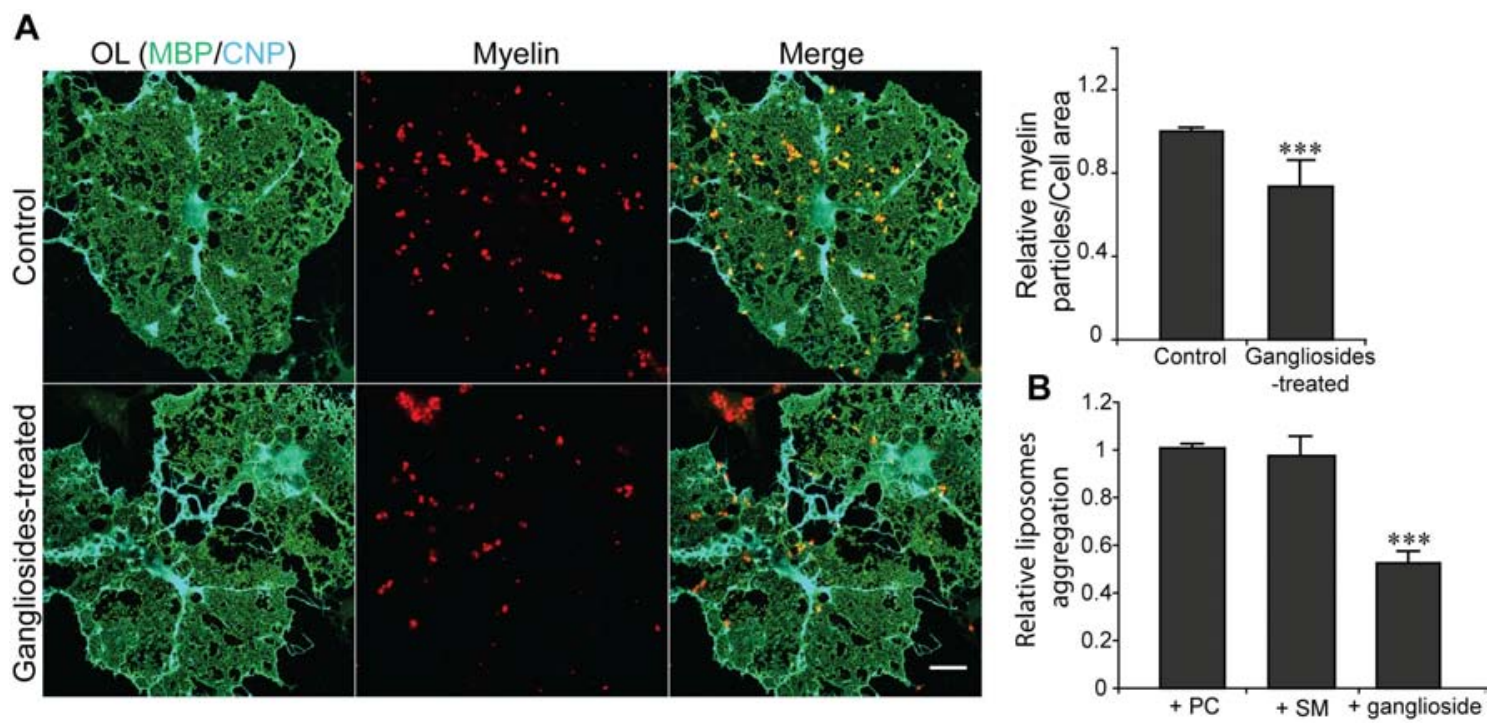

Figure 3.33: Exogenous administration of gangliosides reduces adhesiveness of myelinlike membrane. (A) Total brain gangliosides ( $80 \mu \mathrm{M})$ coupled to defatted BSA were loaded to fully differentiated oligodendrocytes for $1 \mathrm{hr}$ at $37{ }^{\circ} \mathrm{C}$. After extensive washing with PBS, binding assay was performed. Treatment of cells with gangliosides resulted in a decrease in myelin particles binding ( $\mathrm{n}=6,{ }^{* * *} p<0.001 ; t$-test). (B) Exogenous lipids were added to myelin lipids to obtain $10 \%$ of total concentration and liposome aggregation assay was performed. Liposomes that contained gangliosides revealed less aggregation in contrast to sphingomyelin (SM) and phosphatidylcholine (PC) -incorporated vesicles $\left(n=4{ }^{* \star *} p<0.001\right.$; one-way ANOVA)

Down-regulation of sialic acid is essential for myelination in the CNS (Fewou et al., 2007). The polymer of sialic acid, PSA, is the prominent form of this sugar in producing repulsive effect on membranes (Rutishauser et al., 1990). The down-regulation of PSA during oligodendrocyte differentiation has been reported (Trotter et al., 1989; Bartsch et al., 1990). This polymer is biosynthesized by polysialyltransferases which include ST8Sia II (STX) and ST8Sia IV (PST). The main enzyme in oligodendrocyte is, however, PST (Stoykova et al., 2001). We found a reduction in the expression of this enzyme after OLs enter differentiation stage. Western blot analysis indicated a decrease in PST level for cells at 
Figure 3.34: Polysialyl-transferase (ST8SialV) is down-regulated during OL maturation. Oligodendrocyte lysates from cells at day 1,3 and 5 were analyzed for the expression levels of their main polysialyl-transferase, ST8SialV, using western blotting. An obvious reduction in the level of this protein is shown during the cell maturation.

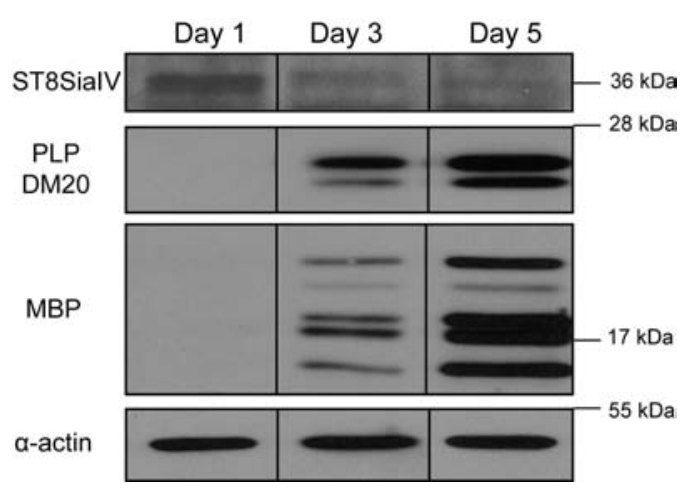

day 3 and 5 compared to day 1 (Figure 3.34). The mice expressing this enzyme in mature oligodendrocytes (PLP-PST) have been described before. These mice develop myelin with structural abnormalities like redundant myelin and axonal degeneration (Fewou et al., 2007). We performed electron microscopic analysis of the optic nerve from these transgenic mice. Conventional embedding preparation of nerve revealed more lamella splitting for these animals compared to the controls (Figure 3.35). We also carried out the myelinmyelin interaction assay in vitro for these mice. We observed a significant reduction in the interaction between PST-transgenic myelin particles compared to WT myelin (Figure 3.36). These data suggest a repulsive effect of PSA on myelin adhesion in vivo and in vitro.

\subsubsection{PLP achieves its function upon sialic acid removal}

Having identified an adhesive role for PLP in myelin membrane, we asked whether we can induce myelin particle interaction with a non-myelin membrane upon expression of this protein. We used PtK2 cell line as a model system. We transfected these cells with PLP plasmid for $20 \mathrm{hr}$ and performed the particle binding assay. However, a subtle difference was detected for PLP-expressing cells compared to the controls. Interestingly, when we treated cells with sialidase, a considerable increase in myelin particle interaction was observed for cells which expressed PLP (Figure 3.37 A, B). This finding suggests that adhesive function of PLP depends on sialic acid removal from the membrane, highlighting the importance of glycocalyx elimination during myelin membrane compaction in the CNS. 

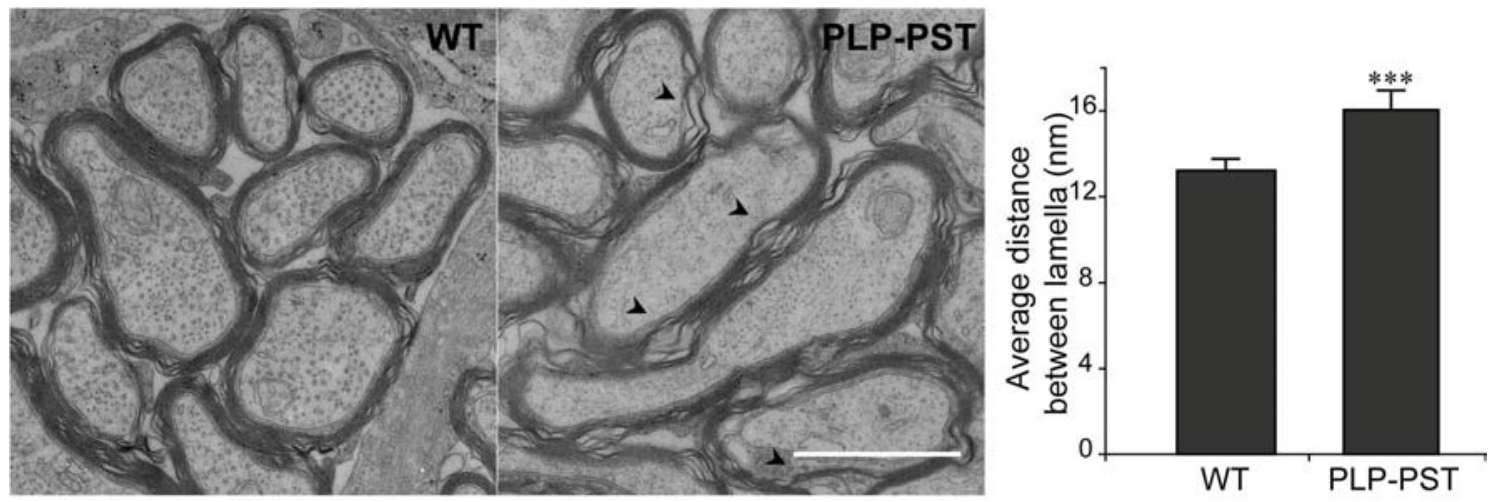

Figure 3.35: PSA-expressing oligodendrocytes produce less stable myelin. Optic nerves from 5-month-old mice were prepared by conventional embedding fixation. Electron micrographs of the samples were obtained and the average distance between myelin lamella was measured. An increase in myelin lamella splitting (arrowheads) occurred for samples from PLP-PST mice compared to WT ( $\mathrm{n}=15$ images from 3 animals, ${ }^{* *} p<0.001 ; t$-test). Scale bar, $1 \mu \mathrm{m}$.
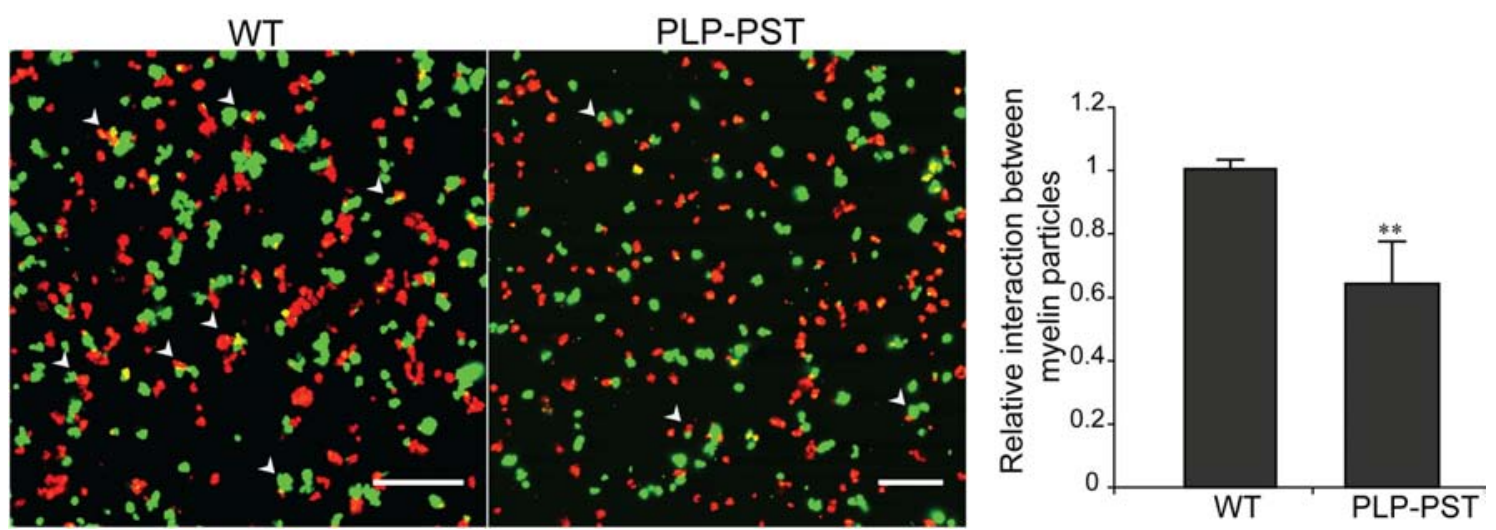

Figure 3.36: PSA expression reduces the interaction between myelin particles. Myelin particles were labeled with two different PKH dyes. After incubation for 1 hour at room temperature, the mixture of particles was applied onto coverslips and then fixed. A higher interaction (arrowheads) between WT particles was detected compared to the PLP-PST myelin. ( $n=3,{ }^{* *} p<0.01$; $t$-test). Scale bar, $20 \mu \mathrm{m}$. 

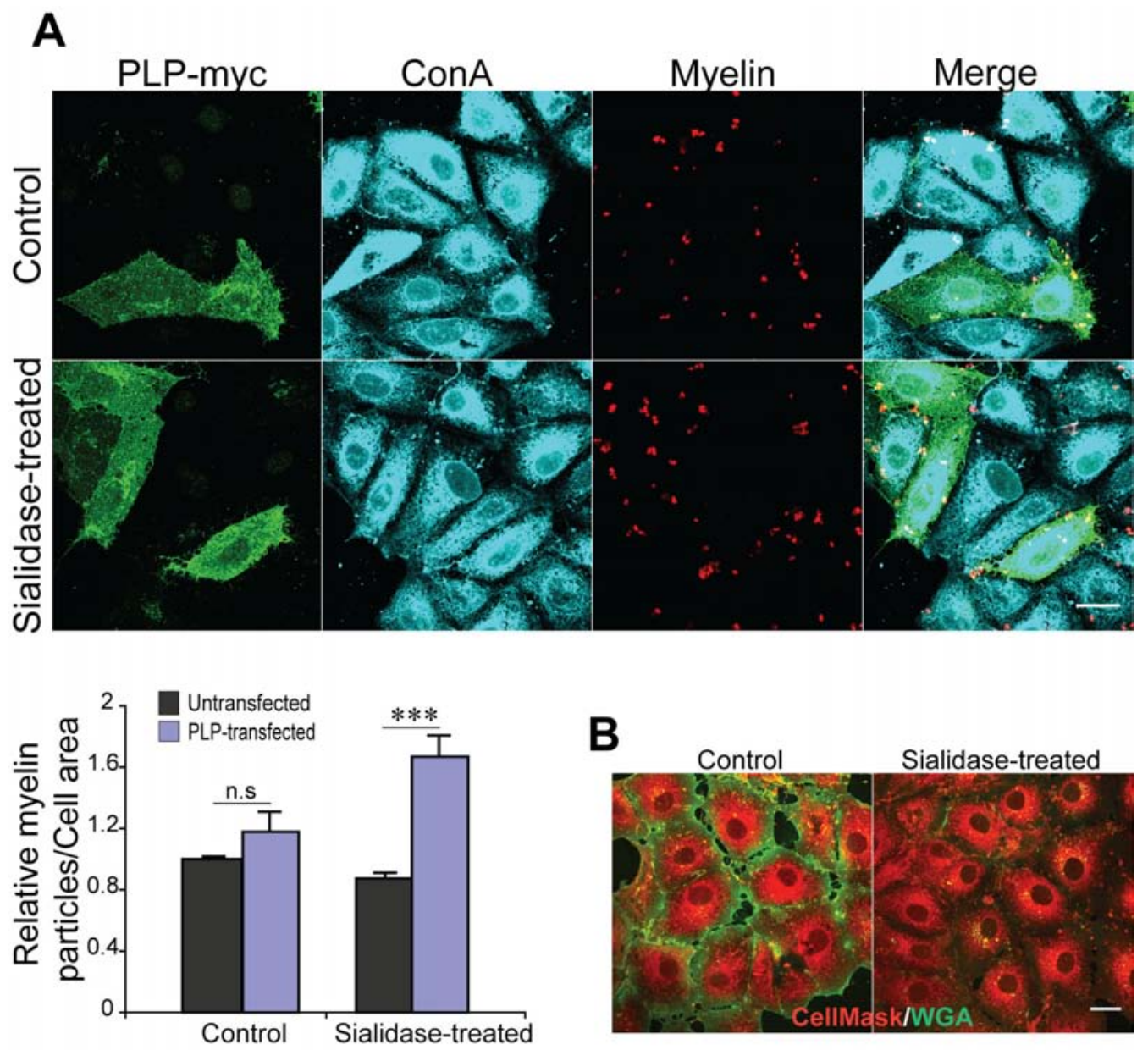

Figure 3.37: PLP expression and sialic acid removal trigger myelin particle binding to nonmyelin membranes. (A) PtK2 cells were transfected with PLP-myc plasmid for $20 \mathrm{hr}$. The cells were subsequently treated with $5 \mathrm{U} / \mathrm{ml}$ sialidase or control buffer for $1 \mathrm{hr}$ at $37{ }^{\circ} \mathrm{C}$. Addition of myelin particles revealed more binding for sialidase-treated PLP expressing cells. PLP transfected cells which were treated by control buffer did not show more binding compared to the controls ( $\mathrm{n}$ $=3,{ }^{* \star *} p<0.001 ; t$-test). (B) Enzymatic sialic acid removal in PtK2 cells was confirmed by WGA staining. Scale bar, $20 \mu \mathrm{m}$. 


\section{Chapter 4}

\section{Discussion}

\subsection{The function of oligodendrocyte exosomes in the CNS}

\subsubsection{Regulation of oligodendrocyte development}

The development of myelinating oligodendrocytes from their precursors is a complex process which is regulated by intrinsic genetic differentiation program that is controlled partially by an intracellular molecular clock determining the entry of these cells to differentiation phase (Temple and Raff, 1986; Barres et al., 1994; Dugas et al., 2007). This intrinsic program is regulated by various extrinsic factors such as Wnt, Lingo-1 and PSA-NCAM which present within the surrounding microenvironment (Wang et al., 1998; Charles et al., 2000; Mi et al., 2005). After OLs enter differentiation stage, they start to synthesize large amounts of myelin components. However, the assembly of myelin membrane sheaths occurs after the biosynthesis of the major myelin components. In fact, OLs that have produced myelin components but have not started to enwrap axons are found for a relatively short period during the CNS development (Trapp et al., 1997). These premyelinating cells seem to produce different myelin components by distinct mechanisms, which might prevent premature and inappropriate assembly of the membrane. Therefore, it is feasible that the regulation of membrane trafficking is a mechanism to induce myelin membrane assembly at the proper time during development. Indeed, previous studies have shown that neurons influence the trafficking of PLP (Trajkovic et al., 2006; Kippert et al., 2007). A quite large fraction of PLP is transported to late endosomes/lysosomes (LE/Ls) in oligodendrocytes, whereas in the presence of neurons the localization of PLP to LE/Ls is reduced (Trajkovic et al., 2006). These late endosomal multivesicular bodies (MVBs) can either be delivered to the endosomal pathway for lysosomal degradation, or they undergo fusion with the plasma membrane to release their intraluminal vesicles into the extracellular space which 
are called exosomes.

\subsubsection{Exosomes secreted by oligodendrocytes inhibit myelination process}

Exosomes are a new type of microvesicles which are produced by different types of cells (Simons and Raposo, 2009). The production of exosomes by oligodendrocytes has been also reported (Krämer-Albers et al., 2007). The secretion of PLP-containing exosomes by oligodendrocytes occurs in a ceramide-dependent manner which is different from the ESCRT-mediated exosomes formation (Trajkovic et al., 2008). Nevertheless, the physiological function of exosomes secreted by oligodendrocytes still remains obscure. Here we investigated the role of oligodendrocyte-derived exosomes during the axon-glia interaction. Our data demonstrated that exosomes reduce OLs surface expansion and consequently inhibit myelination. Moreover, we found that a factor(s) from conditioned neuronal medium reduces exosome release by OLs. Based on our findings, we propose a model where in the absence of neuronal signals, exosomes secreted by OLs maintain these cells in premyelinating stage. However, neuronal factors reduce exosome release by OLs and remove the auto-inhibitory function of these vesicles. Therefore, the inhibitory effect of neuronal signals on exosome release seems to be a positive mechanism to enhance myelination.

\subsubsection{Exosomes inhibit terminal differentiation of OLs to become myelinating cells}

Exosomes do not affect the differentiation of oligodendrocytes from precursor cells; instead, they appear to regulate the growth properties of these cells. Among the various factors that have been reported to affect the life cycle of oligodendrocytes, many of them influence the switching of proliferating OPCs into post-mitotic mature oligodendrocytes (Chong and Chan, 2010). Many of these cues are produced by neurons and seem to reduce the differentiation of oligodendrocytes rather than enhancing it. For instance, PSA-NCAM expressed on the axonal surface and the Notch receptor Jagged1 inhibit OPCs differentiation (Wang et al., 1998; Charles et al., 2000). Other factors that prevent OPCs differentiation are the Wnt signalling pathway, Lingo-1 and the connective tissue growth factor (CTGF) (Mi et al., 2005; Stritt et al., 2009; Ye et al., 2009)). In chronic MS lesions the activation of these pathways might be responsible for the differentiation obstruct that subsequently 
results in inefficient remyelination (Franklin and Ffrench-Constant, 2008). In addition, premyelinating oligodendrocytes have been detected in chronic MS lesions that extend multiple myelin components-positive processes (Chang et al., 2002). These cells have entered the differentiation phase, but failed to remyelinate lesion, probably because they are not receiving the proper signals from the chronically demyelinated axons. Pre-myelinating cells are not normally present in the healthy adult brain, but rather they appear for a relatively short period during CNS development (Trapp et al., 1997). It is feasible that neuronal signals reduce the generation of MVBs and thereby the biogenesis of new exosomes at later stages of oligodendrocyte development. It is also possible that microvesicles are generated directly from the blebbing of plasma membrane into the extracellular space. Interestingly, the premyelinating cells which have been observed in chronic MS lesions include large perikarya positive for PLP (Chang et al., 2002). It will be interesting to find out whether this PLP fraction accumulates in late endosomes/lysosomes and is secreted via exosomes in these premyelinating oligodendrocytes.

\subsubsection{Exosomes influence OLs through a common signalling pathway triggering cell retraction}

The signals responsible for the regulation of exosome release are unknown, but they may lead to alteration intracellular $\mathrm{Ca}^{2+}$ levels that are known to increase exosomal secretion (Krämer-Albers et al., 2007). In turn, these vesicles might induce a variety of signalling cascades in the target cells. We found that the effect of exosomes on OLs is mediated by RhoA signalling. This pathway has been described to regulate OLs branching (Kippert et al., 2009). Inhibition of RhoA function is essential for OLs to extend their processes during myelination. Exosomes seem to employ this common signalling pathway to induce OLs retraction. Src family kinase in particularly Fyn protein have been described to play a role in myelination of the CNS (Sperber et al., 2001). We also found the involvement of Fyn protein during OLs treatment by exosomes. This protein is known to control RhoA signalling pathway in oligodendrocytes. The activity of this protein is regulated by two different phosphorylation sites. Whereas phosphorylation of tyrosine 420 increases Fyn activity, the addition of a phosphate group to tyrosine 531 inhibits the activity. Therefore, numerous factors modulate Fyn activity by changing its phosphorylation pattern. Exosomes inhibit Fyn activity by reducing the phosphorylation of tyrosine 420 whereas they do not affect the phosphorylation level of tyrosine 531. The activity of Fyn to regulate 
myelination by OLs is connected to another kinase, focal adhesion kinase (FAK). This protein is described to mediate integrin signalling in oligodendrocytes which consequently results in myelination in response to ECM factors (Câmara et al., 2009; Forrest et al., 2009). Exosome-induced alteration of FAK phosphorylation revealed the involvement of this protein in the process. We observed that exosomes reduce autophosphorylation of FAK (Y397). Therefore, we conclude that by inactivation of FAK and Fyn proteins, exosomes activate the RhoA cascade which subsequently induces oligodendroglial cell retraction.

\subsubsection{Exosomes and myelin debris have the same effect on OLs}

It is interesting to compare the effects of exosomes to myelin debris released upon demyelination. Myelin debris has a great inhibitory effect on oligodendrocyte differentiation unless they are removed from the extracellular space by phagocytic cells (Miller, 1999; Kotter et al., 2001, 2006). Injection of exogenous myelin in regions of experimentally induced focal demyelination leads to inhibition of remyelination by preventing OPC differentiation (Kotter et al., 2006). Interestingly, this inhibitory effect of myelin on OL differentiation is mediated by Fyn-RhoA-ROCK signalling (Baer et al., 2009). These observations show that so far unknown molecules presented by both myelin debris and exosomes regulate remarkably similar processes by a common signalling cascade. An alternative explanation is that the myelin membrane proteins are only cargo and another unidentified protein mediates this effect. Candidate molecules are such as Lingo- 1 that activates the RhoA-ROCK pathway and reduces Fyn expression and activation (Mi et al., 2005). Also, Netrin-1 has been described to modulate oligodendrocyte process branching and membrane extension via Fyn and RhoA pathway (Rajasekharan et al., 2009). It is not clear why there are so many inhibitory molecules to control myelin membrane formation and assembly in the CNS, whereas in the PNS many inductive factors have been reported to regulate myelination process by Schwann cells. One possible reason for this could be the complex task of coordinating the biosynthesis of up to 40 myelin fragments on different axons by one oligodendrocyte. Therefore, the removal of inhibitory factors from the oligodendrocyte microenvironment may trigger the myelination of all processes at the same time. 


\subsection{Myelin adhesion and compaction in central nervous system}

\subsubsection{Myelin compaction and a new model system for its assessment}

One defining characteristic of myelin is its compact structure, which is achieved by the close adhesion of the different membrane layers. The interaction between layers in cytoplasmic sides is known to be mediated by MBP function to generate the major dense line. However, the molecular mechanisms that generate the intraperiod line (IPL) which is localized between the outer leaflets of the myelin membrane are poorly understood. The major obstacle to investigate this structure is technical limitation. The information so far on myelin compaction are mainly based on electron microscopy studies of mutant animals. However, these approaches have not clearly elucidated the mechanism of myelin compaction in the extracellular space.

Oligodendrocytes are known to produce myelin membrane in vitro. In culture, OPCs differentiate into mature OLs in the absence of neuronal signals and produce large amounts of membrane surronding their perikarya. The morphological and biochemical characterization of this membrane reveals a high similarity to myelin structure in vivo. The protein markers for the compact and non-compact areas are nicely separated in this myelin-like membrane (Aggarwal et al., 2011b). Therefore, this membrane can be a useful model for structural studies regarding CNS myelin in the cell culture system. Taking advantage of this model we established a biding assay between purified myelin particles and oligodendrocytes in culture to mimic the interaction between two myelin membranes in vitro. Interestingly, the interaction between myelin and oligodendrocytes seems to be specific, since these particles have low affinity towards other glial cells. Therefore, we introduce this system as a model to analyse the possible molecular mechanisms involved in myelin compaction.

\subsubsection{The role of PLP in myelin adhesion and compaction}

It has been proposed that the interaction between hydrophilic extracellular domains of PLP may be responsible for tight opposition of membrane sheaths enwrapping axons that leads to the compaction of myelin layers (Weimbs and Stoffel, 1992). To address this question two independent groups have designed mice lacking the plp gene. Boison and colleague generated a $P L P$-knockout mouse, and showed that axons are loosely en- 
wrapped by myelin, displaying wide extracellular spaces between lamella. Despite the abnormality in myelin compaction, these mice were fertile and had a normal life-span without noticeable behavioural symptoms (Boison et al., 1995). In contrast, Klugmann and colleague generated another PLP/DM20-knockout mice (KPLP) which revealed normal myelin biosynthesis and assembly by oligodendrocytes. These results challenged the previous report that proposed an adhesive role for PLP during myelin assembly, suggesting an engagement of its homophilic interaction to form struts to stabilize the IPL (Klugmann et al., 1997). These animals did not show a clear loss of oligodendrocytes and displayed normal myelin synthesis and assembly suggesting that PLP is not essential for myelination in the CNS (Klugmann et al., 1997; Rosenbluth et al., 2006; Möbius et al., 2008). However, normal myelination in PLP-null mice was followed by progression of a widespread axonal swelling and degeneration. This axonal swelling has been shown to be a consequence of the absence of PLP and not of abnormal myelin morphology, which also appears in shiverer mice without inducing the same pathological symptoms (Griffiths et al., 1998b).

Using myelin and oligodendrocytes from KPLP mice we investigated the function of PLP during the interaction between myelin particles and primary oligodendrocytes. We found a decrease in myelin particles binding to OLs in the absence of PLP. Further, the adhesion between myelin particles was increased in the presence of PLP. We also found more aggregation between liposomes prepared from WT myelin in comparison to those from KPLP myelin. This is consistent with the observation that PLP induces rolling, but not fusion of lipid bilayer, indicating the capability of this protein in creating myelin-like structures in vitro (Palaniyar et al., 1998). Furthermore, PLP has been reported to induce PC/cholesterol vesicle aggregation, which is abolished upon disulfide bonds cleavage and methylation of carboxyl group in the protein (Bizzozero and Howard, 2002). This observation has indicated a potent role of PLP in neutral vesicle clustering that is dependent on the protein conformation. Our findings together with previous data suggest an adhesive role for PLP protein in vitro, which proposes that even if this protein is not necessary for myelin biogenesis, it is required for stability and maintenance of myelin layers in compacted area.

\subsubsection{The involvement of PLP in supporting the physical integrity of CNS myelin}

If PLP has adhesive property, it most likely increases the physical stability of myelin. To examine this, the size distribution of myelin particles was analyzed which displayed a 
tendency toward larger sizes for WT myelin compared to KPLP. This shows the fragility of myelin in the absence of this protein. The yield of myelin preparation from PLP null mice is also less than WT. Additionally, the mature myelin from PLP knockout mice is more fragmented throughout the preparation which indicates a lower physical stability of this membrane (Jurevics et al., 2003). Single-particle force spectroscopy revealed stronger interaction between myelin membranes in the presence of PLP. Also, a fluctuation assay indicated an increase in myelin flexibility in the absence of PLP. Together these observations indicate the lower physical stability of KPLP myelin, supporting the structural function of this protein in stabilizing the integriy of myelin membrane. We also analyzed the average distance between lamella in KPLP myelin from optic nerve using electron microscopy. Embedding fixation of nerve preparation revealed more splitting in myelin lamella for KPLP compared to the control. These data are consistent with the observation that have shown more interalamellar space and looser myelin in $P L P$-null mice (Rosenbluth et al., 2006). In addition, it has been shown that myelin sheaths of compact area appeared split into two leaflets and IPL were unfused with more extracellular space than wild-type mice (Rosenbluth et al., 2006). Nevertheless, these myelin split lamella have not been observed upon cryofixation and freeze substitution of samples with careful tissue processing. Based on this observation, it has been speculated that the split lamella may correspond to fixation artifact during sample preparation and not occurs in $P L P$-null mice in vivo (Möbius et al., 2008). However, even if this splitting occurs during sample preparation it shows a greater fragility of myelin in the absence of PLP, which demonstrates the structural role of PLP in the stabilization of myelin membrane integrity. It is, therefore, possible that myelin is indeed of normal ultrastructure in a living animal lacking PLP as suggested by Klugmann and colleague (Klugmann et al., 1997).

The importance of PLP in myelin integrity has also become clear from mice deficient for PLP together with other myelin components. OSP/claudin-11 and PLP are structurally related and are tetraspan proteins. Generating mice deficient for both PLP and OSP/claudin-11, have produced severe neurological deficits with disrupted myelin compaction of smaller axonal calibres compared to the single knockouts (Chow et al., 2005). This suggests that these proteins have compensatory function for each other as the absence of both of them leads to severe deficits. Also, in the mice deficient for PLP and galactocerbrosides a large number of axons in the ventral spinal white matter and the optic nerve were unmyelinated (Coetzee et al., 1999). These observations indicate the involve- 
ment of PLP and galactolipids in maintenance of myelin integrity. They also suggest that a series of complex interaction may be required for formation of IPL and myelin compaction.

\subsubsection{Oligodendrocyte glycocalyx is reduced during their maturation}

The adhesion process between two membranes is controlled by expression of adhesion receptors, intrinsic activity of these molecules, their organization in the cell membrane, and non-specific process such as glycocalyx removal (Vitte et al., 2005). In general, cells are surrounded by a carbohydrate-rich layer, which collectively called glycocalyx (Foa et al., 1996). Despite specific interactions between glycocalyx elements such as interaction of integrins and syndecans to ECM components (Weinbaum et al., 2007), cells use the glycocalyx to modulate their adhesiveness. Presence of glycocalyx impedes adhesion and, therefore, its removal is required for establishment of efficient interaction between two membranes. Here, we showed a downregulation of glycoconjugate molecules from the surface of oligodendrocytes during maturation which seems to be a mechanism to increase myelin membrane adhesion. Using different lectins, we showed that numerous types of sugars are reduced from fully differentiated OLs. Accordingly, lectin staining for adult brain clearly revealed a negative correlation between lectin binding and myelination. The major molecule responsible for repulsive inhibition of cell adhesion is sialic acid. Due to its negative charge, sialic acid induces a non-specific repulsive force between membranes. Mature oligodendrocytes and myelinated areas in the adult brain had low amounts of this molecule. Additionally, enzymatic removal of sialic acid increased myelin-like membrane adhesiveness which supports its role in myelin adhesion and compaction. Interestingly, a reduction in myelinated axons in sialin (lysosomal sialic acid transporter) deficient mice has been described (Prolo et al., 2009). This might support the necessity of sialic acid removal in the myelination process of the CNS .

\subsubsection{The mechanisms involved in glycocalyx removal from OLs surface}

The reduction of glycocalyx from the myelin surface could be regulated by three possible mechanisms; down-regulation of the glycocalyx synthesis machinery, enzymatic removal and glycoproteins exclusion from myelin compact area. Transcriptome analysis of oligodendrocytes has revealed a striking reduction in the expression levels of many enzymes involved in glyco-conjugate synthesis during differentiation (Dugas et al., 2006). We also 
found a reduction in sialic acid synthesis using metabolic incorporation labeling during maturation of oligodendrocytes. This down-regulation of the synthesis machinery might be an intrinsic program for cells to reduce their carbohydrate production after entering the differentiation phase. Indeed, proliferating oligodendrocyte precursors contain many glycocalyx components such as NG2 proteoglycan that are eliminated in mature cells (Nishiyama et al., 2009). Another feasible mechanism for the glycocalyx removal from myelin is enzymatic degradation. The main evidence especially regarding sialic acid removal is provided by finding the intrinsic sialidase activity of myelin. Myelin prepared from rat brain shows intrinsic sialidase activity, that is elevated during the period of active myelination (Saito and Yu, 1986, 1993). In addition, a correlation between sialidase activity and MBP distribution has been described in adult mice brains, which indicates the importance of myelin associated sialidase in myelin maintenance and stability (Minami et al., 2011). This is consistent with our observation that showed low amounts of sialic acids in myelinated areas. One substrate of myelin sialidase can be gangliosides. These lipids exist in myelin as a minor species and are requisite for long-term integrity of axonmyelin interaction (Cochran et al., 1982). Oligodendrocytes are able to synthesize many brain types of gangliosides (Lopez and Schnaar, 2009). However, in mature myelin, many of these lipids are absent and GM1 is the main type (Cochran et al., 1982). Whether selective incorporation of these lipids into myelin or enzymatic removal by sialidase, results in a low density of gangliosides in mature myelin, still remain to be proven ( $\mathrm{Yu}$ et al., 1989). The fact that GM1 as the main ganglioside in mature myelin is resistant to degradation by sialidase ( $\mathrm{Li}$ et al., 2001), might supports the role of this enzyme in removing other gangliosides from myelin. MBP function in myelin can also reduce the glycoprotein content of myelin compact area. This protein acts as a barrier and prevents the entry of proteins with large cytosolic domain to compact region (Aggarwal et al., 2011b). Most of these proteins are, indeed, glycoproteins which are restricted to the non-compact region in mature myelin. Therefore, by reducing glycoprotein content, MBP might indirectly facilitates myelin adhesion in extracellular leaflets. This is interesting since this protein orchestrates the interaction between membranes in cytoplasmic sides. Our observation that myelin particles bound more to WT cells than shiverer, which do not have MBP, also supports this idea. Interestingly, introducing of MAG to compacted region also reduced myelin binding, pointing to the necessity of exclusion of glycoproteins by the size barrier function of MBP during myelin development. 


\subsubsection{Down-regulation of sialic acid content is necessary for myelin compaction}

We found that enzymatic removal of sialic acid in both myelin-like and non-myelin membranes increases their adhesiveness towards myelin particles. Furthermore, exogenous incorporation of gangliosides, as a major source of sialic acid, attenuated myelin adhesion. Interestingly, the reduction of sialic acid polymer, PSA, from myelinating cells is necessary for efficient myelination in the CNS. Transgenic mice expressing PSA (PLP-PST) in mature oligodendrocytes develop myelin structural abnormalities such as redundant myelin, axonal and vacular degeneration (Fewou et al., 2007). By analyzing the optic nerve from PLP-PST mice we revealed more splitting of myelin lamella which indicates less stability of this myelin. Moreover, myelin particles from PLP-PST mice exhibited less adhesion in comparison to WT. Therefore, we suggest that the reduction of PSA from mature myelin might be necessary for the tight adhesion of this membrane from the extracellular sides to organize a compacted structure. Post-translationally addition of PSA to proteins plays important roles in the CNS. PSA-NCAM, for example, is involved in axonal pathfinding and branching, cell migration and synaptic plasticity in the CNS (Charles et al., 2002). To be myelinated, axons eliminate PSA-NCAM from their surface (Jakovcevski et al., 2007), suggesting the repulsive effect of PSA on myelinating glia to approach the axons. Additionally, re-expression of PSA on the surface of demyelinated axons possibly prevents remyelination (Charles et al., 2000, 2002). This may suggests a possible molecular mechanism responsible for a partially remyelination failure in multiple sclerosis. 


\section{Chapter 5}

\section{Summary and conclusion}

Previous studies have reported that oligodendrocytes produce relatively large amounts of exosomes, but the physiological role of these vesicles has remained unclear. Here, we investigated the function of oligodendrocyte-derived exosomes using a cell culture system. In summary, we showed that oligodendrocyte secrete exosome-like vesicles that seem to have an auto-inhibitory effect by reducing oligodendrocyte surface growth. Interestingly, we found that factors from the neuronal conditioned medium reduce the secretion of exosome-like vesicles from oligodendrocytes. Based on these observations, we propose a model in which neurons regulate terminal differentiation of oligodendrocytes by controlling the release of auto-inhibitory exosomes from these myelinating cells (Figure 5.1).

We also found that the effect of exosomes on OLs is mediated by inactivation of FAK-Fyn signalling which leads to activation of RhoA-ROCK pathway. This cascade has been described to regulate OLs process branching and its inhibition is essential for OLs to extend their branches. Therefore, exosomes seem to employ this common signalling pathway to induce OLs retraction. In conclusion, our results provide new evidence for small and diffusible oligodendroglial-derived vesicular carriers within the extracellular space that have inhibitory properties on cellular growth. We propose that secretion of these exosomes coordinate myelin membrane biogenesis.

In the second part, we studied the mechanisms responsible for adhesion and compaction of CNS myelin. We established a binding assay to assess the interaction betwen purified myelin particles and primary oligodendrocytes in culture to mimic the interaction between two myelin membranes. Using this model system we investigated the possible role of PLP as a putative adhesion molecule in myelin membrane adhesion. Our data indicated 


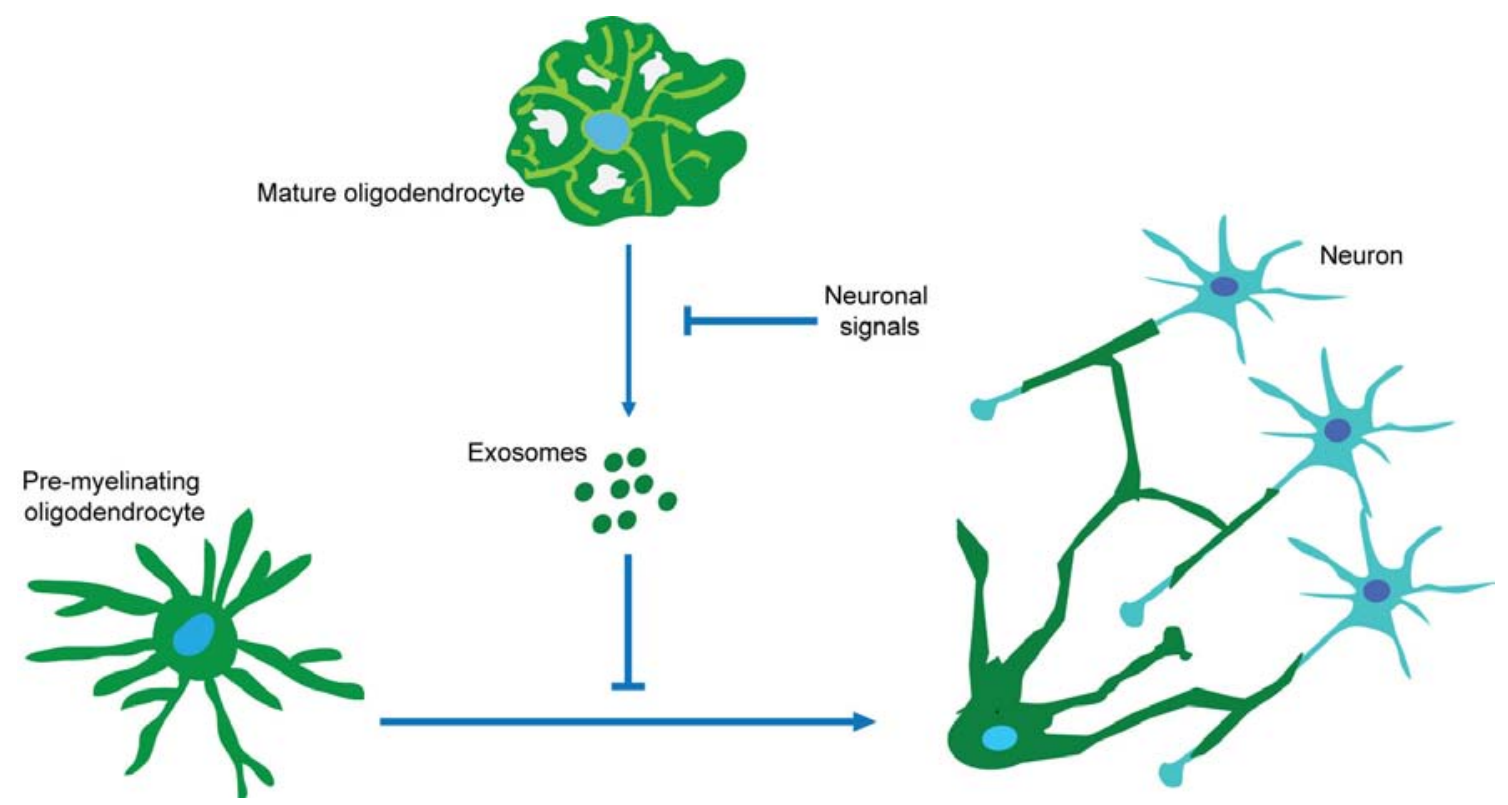

Figure 5.1: A model for the function of oligodendrocyte-derived exosomes. Oligodendrocytes release exosomes which keep cells in premyelinating stage and inhibit myelination. Neuronal signals reduce the secretion of exosomes by OLs and, therefore, remove the inhibitory effect of these vesicles on myelination.

an adhesive properties of this protein in myelin membranes interaction. We confirmed this observation by performing liposome aggregation assay prepared from myelin-derived lipids. We also found the role of PLP in increasing myelin stability using single particle force spectroscopy. Electron microscopy analysis of optic nerve from PLP knockout mice also revealed the involvement of PLP in maintenance and stability of myelin integrity. Taken together we provide evidence for the contribution of PLP in myelin adhesion which increases the physical stability of this membrane.

To establish an efficient membrane adhesion the repulsive molecules should be removed from the surface. Therefore, we explored the involvement of glycocalyx removal in myelin adhesion during maturation of this membrane. We showed a clear reduction in carbohydrates from OLs surface during their maturation by lectin staining. Staining of brain section also revealed a reduction of glycocalyx content from myelinated areas. Furthermore, we found a role of sialic acids as a repulsive molecule in myelin adhesion. Enzymatic removal of sialic acid and its exogenous incorporation support the repulsive effect of this molecule on myelin adhesiveness. In addition, electron microscopic analysis of optic nerve 


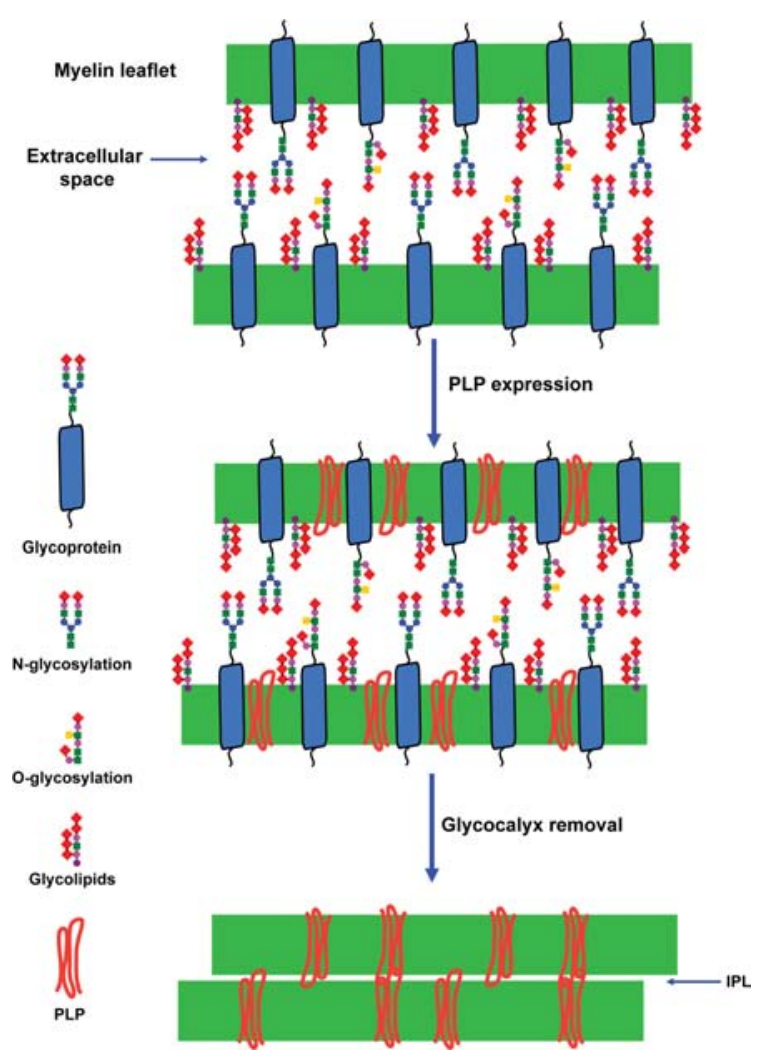

Figure 5.2: A model for myelin adhesion and compaction in CNS. Our data suggests that the removal of the glycocalyx components, mainly sialic acid, along with adhesive function of PLP orchestrate CNS myelin membrane adhesion and compaction in the extracellular sides.

from transgenic mice expressing polysialic acid (PSA) in mature oligodendrocytes as well as myelin particle binding assay from these mice revealed a reduction in myelin adhesion. Our data, therefore, suggest that the removal of glycocalyx is necessary for myelin membrane adhesion. In conclusion, our data suggest that glycocalyx is reduced from myelin membrane during its maturation which leads to exposure of adhesion molecules such as PLP to orchestrate the interaction between myelin membrane layers which subsequently establish myelin compaction (Figure 5.2). 


\section{Bibliography}

Aggarwal, S., Yurlova, L. and Simons, M. (2011a). Central nervous system myelin: structure, synthesis and assembly. Trends in Cell Biology 21, 585-593.

Aggarwal, S., Yurlova, L., Snaidero, N., Reetz, C., Frey, S., Zimmermann, J., Pähler, G., Janshoff, A., Friedrichs, J., Müller, D. J., Goebel, C. and Simons, M. (2011b). A size barrier limits protein diffusion at the cell surface to generate lipid-rich myelin-membrane sheets. Developmental Cell $21,445-456$.

Anitei, M. and Pfeiffer, S. E. (2006). Myelin biogenesis: sorting out protein trafficking. Current Biology 16, R418-R421.

Baer, A. S., Syed, Y. A., Kang, S. U., Mitteregger, D., Vig, R., Ffrench-Constant, C., Franklin, R. J. M., Altmann, F., Lubec, G. and Kotter, M. R. (2009). Myelin-mediated inhibition of oligodendrocyte precursor differentiation can be overcome by pharmacological modulation of Fyn-RhoA and protein kinase C signalling. Brain 132, 465-481.

Barres, B. a. and Barde, Y. (2000). Neuronal and glial cell biology. Current Opinion in Neurobiology $10,642-8$

Barres, B. A., Hart, I. K., Coles, H. S., Burne, J. F., Voyvodic, J. T., Richardson, W. D. and Raff, M. C. (1992). Cell death and control of cell survival in the oligodendrocyte lineage. Cell 70, $31-46$.

Barres, B. A., Lazar, M. A. and Raff, M. C. (1994). A novel role for thyroid-hormone, glucocorticoids and retinoic acid in timing oligodendrocyte development. Development 120, 1097-1108.

Barres, B. A. and Raff, M. C. (1999). Axonal control of oligodendrocyte development. The Journal of Cell Biology 147, 1123-1128.

Bartsch, U., Kirchhoff, F. and Schachner, M. (1990). Highly sialylated N-CAM is expressed in adult mouse optic nerve and retina. Journal of Neurocytology 19, 550-565.

Bauer, N. G., Richter-Landsberg, C. and Ffrench-Constant, C. (2009). Role of the oligodendroglial cytoskeleton in differentiation and myelination. Glia 57, 1691-1705. 
Baumann, N. and Pham-Dinh, D. (2001). Biology of oligodendrocyte and myelin in the mammalian central nervous system. Physiological Reviews 81, 871-927.

Bellingham, S. A., Guo, B. B., Coleman, B. M. and Hill, A. F. (2012). Exosomes: vehicles for the transfer of toxic proteins associated with neurodegenerative diseases? Frontiers in Physiology 3, 124 .

Bernard, C. C., Johns, T. G., Slavin, A., Ichikawa, M., Ewing, C., Liu, J. and Bettadapura, J. (1997). Myelin oligodendrocyte glycoprotein: a novel candidate autoantigen in multiple sclerosis. Journal of Molecular Medicine 75, 77-88.

Bizzozero, O. A. and Howard, T. A. (2002). Myelin proteolipid protein-induced aggregation of lipid vesicles: efficacy of the various molecular species. Neurochemical Research 27, 1269-1277.

Bligh, E. G. and Dyer, W. J. (1959). A rapid method of total lipid extraction and purification. Canadian Journal of Biochemistry and Physiology 37, 911-917.

Bobrie, A., Colombo, M., Raposo, G. and Théry, C. (2011). Exosome secretion : molecular mechanisms and roles in immune responses. Traffic 12, 1-10.

Boggs, J. M., Vail, W. J. and Moscarello, M. A. (1994). Preparation and properties of vesicles of a purified myelin hydrophobic protein and phospholipid. A spin label study. Biochimica et Biophysica Acta 1194, 517-530.

Boison, D., Büssow, H., D’Urso, D., Müller, H. W. and Stoffel, W. (1995). Adhesive properties of proteolipid protein are responsible for the compaction of CNS myelin sheaths. Journal of Neuroscience 15, 5502-5513.

Bosio, A., Binczek, E. and Stoffel, W. (1996). Functional breakdown of the lipid bilayer of the myelin membrane in central and peripheral nervous system by disrupted galactocerebroside synthesis. Proceedings of the National Academy of Sciences of the United States of America 93, 13280-13285.

Bozzali, M. and Wrabetz, L. (2004). Axonal signals and oligodendrocyte differentiation. Neurochemical Research 29, 979-988.

Bradl, M. and Lassmann, H. (2010). Oligodendrocytes: biology and pathology. Acta Neuropathologica 119, 37-53.

Budde, H., Schmitt, S., Fitzner, D., Opitz, L., Salinas-Riester, G. and Simons, M. (2010). Control of oligodendroglial cell number by the miR-17-92 cluster. Development 137, 2127-2132.

Cai, X. D., Golde, T. E. and Younkin, S. G. (1993). Release of excess amyloid beta protein from a mutant amyloid beta protein precursor. Science 259, 514-516. 
Câmara, J., Wang, Z., Nunes-Fonseca, C., Friedman, H. C., Grove, M., Sherman, D. L., Komiyama, N. H., Grant, S. G., Brophy, P. J., Peterson, A. and Ffrench-Constant, C. (2009). Integrinmediated axoglial interactions initiate myelination in the central nervous system. The Journal of Cell Biology 185, 699-712.

Casaccia-Bonnefil, P., Tikoo, R., Kiyokawa, H., Friedrich, V., Chao, M. V. and Koff, A. (1997). Oligodendrocyte precursor differentiation is perturbed in the absence of the cyclin-dependent kinase inhibitor p27Kip1. Genes \& Development 11, 2335-2346.

Chakraborty, C., Nandi, S. and Jana, S. (2005). Prion disease: a deadly disease for protein misfolding. Current Pharmaceutical Biotechnology 6, 167-177.

Chang, A., Tourtellotte, W. W., Rudick, R. and Trapp, B. D. (2002). Premyelinating oligodendrocytes in chronic lesions of multiple sclerosis. The New England Journal of Medicine 346, $165-173$.

Charles, P., Hernandez, M. P., Stankoff, B., Aigrot, M. S., Colin, C., Rougon, G., Zalc, B. and Lubetzki, C. (2000). Negative regulation of central nervous system myelination by polysialylatedneural cell adhesion molecule. Proceedings of the National Academy of Sciences of the United States of America 97, 7585-7590.

Charles, P., Reynolds, R., Seilhean, D., Rougon, G., Aigrot, M. S., Niezgoda, A., Zalc, B. and Lubetzki, C. (2002). Re-expression of PSA-NCAM by demyelinated axons: an inhibitor of remyelination in multiple sclerosis? Brain 125, 1972-1979.

Chen, Y., Wu, H., Wang, S., Koito, H., Li, J., Ye, F., Hoang, J., Escobar, S. S., Gow, A., Arnett, H. A., Trapp, B. D., Karandikar, N. J., Hsieh, J. and Lu, Q. R. (2009). The oligodendrocytespecific G protein-coupled receptor GPR17 is a cell-intrinsic timer of myelination. Nature Neuroscience 12, 1398-1406.

Chernoff, G. F. (1981). Shiverer: an autosomal recessive mutant mouse with myelin deficiency. The Journal of Heredity $72,128$.

Chivet, M., Hemming, F., Pernet-Gallay, K., Fraboulet, S. and Sadoul, R. (2012). Emerging role of neuronal exosomes in the central nervous system. Frontiers in Physiology 3, 145.

Chong, S. Y. C. and Chan, J. R. (2010). Tapping into the glial reservoir: cells committed to remaining uncommitted. The Journal of Cell Biology 188, 305-312.

Chow, E., Mottahedeh, J., Prins, M., Ridder, W., Nusinowitz, S. and Bronstein, J. M. (2005). Disrupted compaction of CNS myelin in an OSP/Claudin-11 and PLP/DM20 double knockout mouse. Molecular and Cellular Neurosciences 29, 405-413. 
Cochran, F. B., Yu, R. K. and Ledeen, R. W. (1982). Myelin gangliosides in vertebrates. Journal of Neurochemistry 39, 773-779.

Cocucci, E., Racchetti, G. and Meldolesi, J. (2009). Shedding microvesicles: artefacts no more. Trends in Cell Biology 19, 43-51.

Coetzee, T., Suzuki, K., Nave, K. A. and Popko, B. (1999). Myelination in the absence of galactolipids and proteolipid proteins. Molecular and Cellular Neurosciences 14, 41-51.

Coman, I., Barbin, G., Charles, P., Zalc, B. and Lubetzki, C. (2005). Axonal signals in central nervous system myelination, demyelination and remyelination. Journal of the Neurological Sciences 233, 67-71.

Curry, F. E. and Adamson, R. H. (2011). Endothelial Glycocalyx: Permeability Barrier and Mechanosensor. Annals of Biomedical Engineering 40, 828-839.

De Castro, F. and Bribián, A. (2005). The molecular orchestra of the migration of oligodendrocyte precursors during development. Brain Research 49, 227-241.

De Gassart, A., Géminard, C., Hoekstra, D. and Vidal, M. (2004). Exosome secretion: the art of reutilizing nonrecycled proteins? Traffic 5, 896-903.

Demerens, C., Stankoff, B., Logak, M., Anglade, P., Allinquant, B., Couraud, F., Zalc, B. and Lubetzki, C. (1996). Induction of myelination in the central nervous system by electrical activity. Proceedings of the National Academy of Sciences of the United States of America 93, 9887-9892.

Denzer, K., Kleijmeer, M. J., Heijnen, H. F., Stoorvogel, W. and Geuze, H. J. (2000). Exosome: from internal vesicle of the multivesicular body to intercellular signaling device. Journal of Cell Science 113 Pt 19, 3365-3374.

Dugas, J. C., Cuellar, T. L., Scholze, A., Ason, B., Ibrahim, A., Emery, B., Zamanian, J. L., Foo, L. C., McManus, M. T. and Barres, B. A. (2010). Dicer1 and miR-219 Are required for normal oligodendrocyte differentiation and myelination. Neuron 65, 597-611.

Dugas, J. C., Ibrahim, A. and Barres, B. A. (2007). A crucial role for p57(Kip2) in the intracellular timer that controls oligodendrocyte differentiation. Journal of Neuroscience 27, 6185-6196.

Dugas, J. C. and Notterpek, L. (2011). MicroRNAs in Oligodendrocyte and Schwann Cell Differentiation. Developmental Neuroscience 5125, 1-7.

Dugas, J. C., Tai, Y. C., Speed, T. P., Ngai, J. and Barres, B. A. (2006). Functional genomic analysis of oligodendrocyte differentiation. Journal of Neuroscience 26, 10967-10983.

Dupree, J. L., Suzuki, K. and Popko, B. (1998). Galactolipids in the formation and function of the myelin sheath. Microscopy Research and Technique 41, 431-440. 
Earl, C., Chantry, A., Mohammad, N. and Glynn, P. (1988). Zinc ions stabilise the association of basic protein with brain myelin membranes. Journal of Neurochemistry $51,718-724$.

Eggeling, C., Ringemann, C., Medda, R., Schwarzmann, G., Sandhoff, K., Polyakova, S., Belov, V. N., Hein, B., Von Middendorff, C., Schönle, A. and Hell, S. W. (2009). Direct observation of the nanoscale dynamics of membrane lipids in a living cell. Nature $45 \%, 1159-62$.

Emery, B. (2010a). Regulation of oligodendrocyte differentiation and myelination. Science 330, $779-782$.

Emery, B. (2010b). Transcriptional and post-transcriptional control of CNS myelination. Current Opinion in Neurobiology 20,601-607.

Erne, B., Sansano, S., Frank, M. and Schaeren-Wiemers, N. (2002). Rafts in adult peripheral nerve myelin contain major structural myelin proteins and myelin and lymphocyte protein (MAL) and CD59 as specific markers. Journal of Neurochemistry 82, 550-562.

Fader, C. M., Savina, A., Sánchez, D. and Colombo, M. I. (2005). Exosome secretion and red cell maturation: Exploring molecular components involved in the docking and fusion of multivesicular bodies in K562 cells. Blood Cells Molecules Diseases 35, 153-157.

Fancy, S. P. J., Chan, J. R., Baranzini, S. E., Franklin, R. J. M. and Rowitch, D. H. (2011). Myelin regeneration: a recapitulation of development? Annual Review of Neuroscience 34, 21-43.

Fernandez, P. A., Tang, D. G., Cheng, L., Prochiantz, A., Mudge, A. W. and Raff, M. C. (2000). Evidence that axon-derived neuregulin promotes oligodendrocyte survival in the developing rat optic nerve. Neuron 28, 81-90.

Février, B. and Raposo, G. (2004). Exosomes: endosomal-derived vesicles shipping extracellular messages. Current Opinion in Cell Biology 16, 415-421.

Fevrier, B., Vilette, D., Archer, F., Loew, D., Faigle, W., Vidal, M., Laude, H. and Raposo, G. (2004). Cells release prions in association with exosomes. Proceedings of the National Academy of Sciences of the United States of America 101, 9683-9688.

Fewou, S. N., Ramakrishnan, H., Büssow, H., Gieselmann, V. and Eckhardt, M. (2007). Downregulation of polysialic acid is required for efficient myelin formation. The Journal of Biological Chemistry 282, 16700-16711.

Finkelstein, E. I., Chao, P.-h. G., Hung, C. T. and Bulinski, J. C. (2007). Electric field-induced polarization of charged cell surface proteins does not determine the direction of galvanotaxis. Cell Motility and the Cytoskeleton 64, 833-846. 
Fitzner, D., Schneider, A., Kippert, A., Möbius, W., Willig, K. I., Hell, S. W., Bunt, G., Gaus, K. and Simons, M. (2006). Myelin basic protein-dependent plasma membrane reorganization in the formation of myelin. The European Molecular Biology Organization Journal 25, 5037-5048.

Flores, A. I., Mallon, B. S., Matsui, T., Ogawa, W., Rosenzweig, A., Okamoto, T. and Macklin, W. B. (2000). Akt-mediated survival of oligodendrocytes induced by neuregulins. Journal of Neuroscience 20, 7622-7630.

Flores, A. I., Narayanan, S. P., Morse, E. N., Shick, H. E., Yin, X., Kidd, G., Avila, R. L., Kirschner, D. A. and Macklin, W. B. (2008). Constitutively active Akt induces enhanced myelination in the CNS. Journal of Neuroscience 28, 7174-7183.

Foa, C., Soler, M., Benoliel, A.-M. and Bongrand, P. (1996). Steric stabilization and cell adhesion. Journal of Materials Science Materials in Medicine 7, 141-148.

Forrest, A. D., Beggs, H. E., Reichardt, L. F., Dupree, J. L., Colello, R. J. and Fuss, B. (2009). Focal adhesion kinase (FAK): A regulator of CNS myelination. Journal of Neuroscience Research 87, 3456-3464.

Frank, M. (2000). MAL, a proteolipid in glycosphingolipid enriched domains: functional implications in myelin and beyond. Progress in Neurobiology 60, 531-544.

Franklin, R. J. M. and Ffrench-Constant, C. (2008). Remyelination in the CNS: from biology to therapy. Nature Reviews Neuroscience 9, 839-855.

Franklin, R. J. M. and Kotter, M. R. (2008). The biology of CNS remyelination: the key to therapeutic advances. Journal of Neurology 255 Suppl, 19-25.

Frühbeis, C., Fröhlich, D. and Krämer-Albers, E.-M. (2012). Emerging roles of exosomes in neuronglia communication. Frontiers in Physiology 3, 119.

Fujimoto, I., Bruses, J. L. and Rutishauser, U. (2001). Regulation of cell adhesion by polysialic acid. Effects on cadherin, immunoglobulin cell adhesion molecule, and integrin function and independence from neural cell adhesion molecule binding or signaling activity. The Journal of Biological Chemistry 276, 31745-31751.

Glynn, P., Chantry, A., Groome, N. and Cuzner, M. L. (1987). Basic protein dissociating from myelin membranes at physiological ionic strength and $\mathrm{pH}$ is cleaved into three major fragments. Journal of Neurochemistry $48,752-759$.

Goto, J., Tezuka, T., Nakazawa, T., Sagara, H. and Yamamoto, T. (2008). Loss of Fyn tyrosine kinase on the C57BL/6 genetic background causes hydrocephalus with defects in oligodendrocyte development. Molecular and Cellular Neurosciences 38, 203-212. 
Greer, J. M., Dyer, C. A., Pakaski, M., Symonowicz, C. and Lees, M. B. (1992). Orientation of the myelin proteolipid protein C-terminus in oligodendroglial membranes. Neurochemical Research 21, 112-121.

Griffiths, I., Klugmann, M., Anderson, T., Thomson, C., Vouyiouklis, D. and Nave, K. A. (1998a). Current concepts of PLP and its role in the nervous system. Microscopy Research and Technique 41, 344-358.

Griffiths, I., Klugmann, M., Anderson, T., Yool, D., Thomson, C., Schwab, M. H., Schneider, A., Zimmermann, F., McCulloch, M., Nadon, N. and Nave, K. A. (1998b). Axonal swellings and degeneration in mice lacking the major proteolipid of myelin. Science 280, 1610-1613.

Heijnen, H. F., Schiel, A. E., Fijnheer, R., Geuze, H. J. and Sixma, J. J. (1999). Activated platelets release two types of membrane vesicles: microvesicles by surface shedding and exosomes derived from exocytosis of multivesicular bodies and alpha-granules. Blood 94, 3791-3799.

Hsu, C., Morohashi, Y., Yoshimura, S.-i., Manrique-Hoyos, N., Jung, S., Lauterbach, M. A., Bakhti, M., Grø nborg, M., Möbius, W., Rhee, J., Barr, F. A. and Simons, M. (2010). Regulation of exosome secretion by Rab35 and its GTPase-activating proteins TBC1D10A-C. The Journal of Cell Biology 189, 223-232.

Hurley, J. H. and Hanson, P. I. (2010). Membrane budding and scission by the ESCRT machinery: it's all in the neck. Nature Reviews Molecular Cell Biology 11, 556-66.

Iero, M., Valenti, R., Huber, V., Filipazzi, P., Parmiani, G., Fais, S. and Rivoltini, L. (2008). Tumour-released exosomes and their implications in cancer immunity. Cell Death and Differentiation $15,80-88$.

Ishibashi, T., Dakin, K. A., Stevens, B., Lee, P. R., Kozlov, S. V., Stewart, C. L. and Fields, R. D. (2006). Astrocytes promote myelination in response to electrical impulses. Neuron 49, 823-832.

Ishibashi, T., Ding, L., Ikenaka, K., Inoue, Y., Miyado, K., Mekada, E. and Baba, H. (2004). Tetraspanin protein CD9 is a novel paranodal component regulating paranodal junctional formation. Journal of Neuroscience 24, 96-102.

Jackman, N., Ishii, A. and Bansal, R. (2009). Oligodendrocyte development and myelin biogenesis: parsing out the roles of glycosphingolipids. Physiology 24, 290-7.

Jahn, O., Tenzer, S. and Werner, H. B. (2009). Myelin Proteomics: Molecular Anatomy of an Insulating Sheath. Molecular Neurobiology 40, 55-72.

Jakovcevski, I., Filipovic, R., Mo, Z., Rakic, S. and Zecevic, N. (2009). Oligodendrocyte development and the onset of myelination in the human fetal brain. Frontiers in Neuroanatomy 3, 15. 
Jakovcevski, I., Mo, Z. and Zecevic, N. (2007). Down-regulation of the axonal polysialic acidneural cell adhesion molecule expression coincides with the onset of myelination in the human fetal forebrain. Neuroscience 149, 328-337.

Jessen, K. (2004). Glial cells. The International Journal of Biochemistry Cell Biology 36, 18611867.

Johnson, C. P. and Chapman, E. R. (2010). Otoferlin is a calcium sensor that directly regulates SNARE-mediated membrane fusion. The Journal of Cell Biology 191, 187-197.

Johnson, C. P., Fujimoto, I., Rutishauser, U. and Leckband, D. E. (2005). Direct evidence that neural cell adhesion molecule (NCAM) polysialylation increases intermembrane repulsion and abrogates adhesion. The Journal of Biological Chemistry 280, 137-145.

Johnstone, R. M. (2006). Exosomes biological significance: A concise review. Blood Cells Molecules Diseases 36, 315-321.

Jurevics, H., Hostettler, J., Sammond, D. W., Nave, K.-A., Toews, A. D. and Morell, P. (2003). Normal metabolism but different physical properties of myelin from mice deficient in proteolipid protein. Journal of Neuroscience Research 71, 826-834.

Keller, S., Sanderson, M. P., Stoeck, A. and Altevogt, P. (2006). Exosomes: from biogenesis and secretion to biological function. Immunology Letters 10\%, 102-108.

Kippert, A., Fitzner, D., Helenius, J. and Simons, M. (2009). Actomyosin contractility controls cell surface area of oligodendrocytes. BMC Cell Biology 10, 71.

Kippert, A., Trajkovic, K., Rajendran, L., Ries, J. and Simons, M. (2007). Rho regulates membrane transport in the endocytic pathway to control plasma membrane specialization in oligodendroglial cells. Journal of Neuroscience 2\%, 3560-3570.

Kitada, M., Kuroda, Y. and Dezawa, M. (2011). Lectins as a tool for detecting neural stem/progenitor cells in the adult mouse brain. Anatomical Record 294, 305-321.

Klugmann, M., Schwab, M. H., Pühlhofer, A., Schneider, A., Zimmermann, F., Griffiths, I. R. and Nave, K. A. (1997). Assembly of CNS myelin in the absence of proteolipid protein. Neuron 18 , $59-70$.

Kokubo, H., Lemere, C. A. and Yamaguchi, H. (2000). Localization of flotillins in human brain and their accumulation with the progression of Alzheimer's disease pathology. Neuroscience Letters 290, 93-96.

Kotter, M. R., Li, W.-W., Zhao, C. and Franklin, R. J. M. (2006). Myelin impairs CNS remyelination by inhibiting oligodendrocyte precursor cell differentiation. Journal of Neuroscience 26, $328-332$. 
Kotter, M. R., Setzu, A., Sim, F. J., Van Rooijen, N. and Franklin, R. J. (2001). Macrophage depletion impairs oligodendrocyte remyelination following lysolecithin-induced demyelination. Glia 35, 204-212.

Krämer, E. M., Schardt, A. and Nave, K. A. (2001). Membrane traffic in myelinating oligodendrocytes. Microscopy Research and Technique 52, 656-671.

Krämer-Albers, E.-M., Bretz, N., Tenzer, S., Winterstein, C., Möbius, W., Berger, H., Nave, K.-A., Schild, H. and Trotter, J. (2007). Oligodendrocytes secrete exosomes containing major myelin and stress-protective proteins: Trophic support for axons? Proteomics Clinical Applications 1, $1446-1461$.

Kuhlmann-Krieg, S., Sommer, I. and Schachner, M. (1988). Ultrastructural features of cultured oligodendrocytes expressing stage-specific cell-surface antigens. Brain Research 467, 269-280.

Lai, C. P.-K. and Breakefield, X. O. (2012). Role of Exosomes/Microvesicles in the Nervous System and Use in Emerging Therapies. Frontiers in Physiology 3, 228.

Lakkaraju, A. and Rodriguez-Boulan, E. (2008). Itinerant exosomes: emerging roles in cell and tissue polarity. Trends in Cell Biology 18, 199-209.

Lappe-Siefke, C., Goebbels, S., Gravel, M., Nicksch, E., Lee, J., Braun, P. E., Griffiths, I. R. and Nave, K.-A. (2003). Disruption of Cnp1 uncouples oligodendroglial functions in axonal support and myelination. Nature Genetics 33, 366-374.

Larocca, J. N. and Norton, W. T. (2007). Isolation of myelin. Current Protocols in Cell Biology Chapter 3, Unit3.25.

Laursen, L. S., Chan, C. W. and Ffrench-Constant, C. (2009). An Integrin-Contactin Complex Regulates CNS Myelination by Differential Fyn Phosphorylation. Journal of Neuroscience 29, 9174-9185.

Li, C., Tropak, M. B., Gerlai, R., Clapoff, S., Abramow-Newerly, W., Trapp, B., Peterson, A. and Roder, J. (1994). Myelination in the absence of myelin-associated glycoprotein. Nature 369, $747-750$.

Li, S. C., Li, Y. T., Moriya, S. and Miyagi, T. (2001). Degradation of G(M1) and G(M2) by mammalian sialidases. The Biochemical Journal 360, 233-237.

Liang, X., Draghi, N. A. and Resh, M. D. (2004). Signaling from integrins to Fyn to Rho family GTPases regulates morphologic differentiation of oligodendrocytes. Journal of Neuroscience 24 , $7140-7149$. 
Linington, C. and Waehneldt, T. V. (1990). Conservation of the carboxyl terminal epitope of myelin proteolipid protein in the tetrapods and lobe-finned fish. Journal of Neurochemistry 54 , $1354-1359$.

Lopez, P. H. H. and Schnaar, R. L. (2009). Gangliosides in cell recognition and membrane protein regulation. Current Opinion in Structural Biology 19, 549-557.

Magyar, J. P., Ebensperger, C., Schaeren-Wiemers, N. and Suter, U. (1997). Myelin and lymphocyte protein (MAL/MVP17/VIP17) and plasmolipin are members of an extended gene family. Gene 189, 269-275.

Maier, O., Hoekstra, D. and Baron, W. (2008). Polarity development in oligodendrocytes: sorting and trafficking of myelin components. Journal of Molecular Neuroscience 35, 35-53.

Mathivanan, S., Ji, H. and Simpson, R. J. (2010). Exosomes: extracellular organelles important in intercellular communication. Journal of Proteomics 73, 1907-1920.

McTigue, D. M. and Tripathi, R. B. (2008). The life, death, and replacement of oligodendrocytes in the adult CNS. Journal of Neurochemistry 10\%, 1-19.

Mi, S., Miller, R. H., Lee, X., Scott, M. L., Shulag-Morskaya, S., Shao, Z., Chang, J., Thill, G., Levesque, M., Zhang, M., Hession, C., Sah, D., Trapp, B., He, Z., Jung, V., McCoy, J. M. and Pepinsky, R. B. (2005). LINGO-1 negatively regulates myelination by oligodendrocytes. Nature Neuroscience $8,745-751$.

Miller, R. H. (1999). Contact with central nervous system myelin inhibits oligodendrocyte progenitor maturation. Developmental Biology 216, 359-368.

Miller, R. H. (2002). Regulation of oligodendrocyte development in the vertebrate CNS. Progress in Neurobiology $67,451-467$.

Minami, A., Shimizu, H., Meguro, Y., Shibata, N., Kanazawa, H., Ikeda, K. and Suzuki, T. (2011). Imaging of sialidase activity in rat brain sections by a highly sensitive fluorescent histochemical method. NeuroImage $58,34-40$.

Miron, V. E., Kuhlmann, T. and Antel, J. P. (2011). Cells of the oligodendroglial lineage, myelination, and remyelination. Biochimica et Biophysica Acta 1812, 184-193.

Mittelbrunn, M. and Sánchez-Madrid, F. (2012). Intercellular communication: diverse structures for exchange of genetic information. Nature Reviews Molecular Cell Biology 13, 328-335.

Möbius, W., Cooper, B., Kaufmann, W. A., Imig, C., Ruhwedel, T., Snaidero, N., Saab, A. S. and Varoqueaux, F. (2010). Electron microscopy of the mouse central nervous system. Methods in Cell Biology 96, 475-512. 
Möbius, W., Patzig, J., Nave, K.-A. and Werner, H. B. (2008). Phylogeny of proteolipid proteins: divergence, constraints, and the evolution of novel functions in myelination and neuroprotection. Neuron Glia Biology 4, 111-127.

Narayanan, S. P., Flores, A. I., Wang, F. and Macklin, W. B. (2009). Akt signals through the mammalian target of rapamycin pathway to regulate CNS myelination. Journal of Neuroscience 29, 6860-6870.

Nave, K.-A. and Trapp, B. D. (2008). Axon-glial signaling and the glial support of axon function. Annual Review of Neuroscience 31, 535-561.

Ndubaku, U. and De Bellard, M. E. (2008). Glial cells: old cells with new twists. Acta Histochemica 110, 182-195.

Niehaus, A. and Trotter, J. (1997). Lectin-binding properties of oligodendrocyte lineage cells aid in defining functionally important surface proteins. Glia 20, 173-183.

Nishiyama, A., Komitova, M., Suzuki, R. and Zhu, X. (2009). Polydendrocytes (NG2 cells): multifunctional cells with lineage plasticity. Nature Reviews Neuroscience 10, 9-22.

Palaniyar, N., Semotok, J. L., Wood, D. D., Moscarello, M. A. and Harauz, G. (1998). Human proteolipid protein (PLP) mediates winding and adhesion of phospholipid membranes but prevents their fusion. Biochimica et Biophysica Acta 1415, 85-100.

Park, S.-K., Miller, R., Krane, I. and Vartanian, T. (2001). The erbB2 gene is required for the development of terminally differentiated spinal cord oligodendrocytes. The Journal of Cell Biology 154, 1245-1258.

Piper, R. C. and Katzmann, D. J. (2007). Biogenesis and function of multivesicular bodies. Annual Review of Cell and Developmental Biology 23, 519-547.

Poltorak, M., Sadoul, R., Keilhauer, G., Landa, C., Fahrig, T. and Schachner, M. (1987). Myelinassociated glycoprotein, a member of the L2/HNK-1 family of neural cell adhesion molecules, is involved in neuron-oligodendrocyte and oligodendrocyte-oligodendrocyte interaction. The Journal of Cell Biology 105, 1893-1899.

Prolo, L. M., Vogel, H. and Reimer, R. J. (2009). The lysosomal sialic acid transporter sialin is required for normal CNS myelination. Journal of Neuroscience 29, 15355-15365.

Quarles, R. H., Ilyas, A. A. and Willison, H. J. (1990). Antibodies to gangliosides and myelin proteins in Guillain-Barré syndrome. Annals of Neurology 27 Suppl, S48-S52.

Quarles, R. H., Macklin, W. B. and Morell, P. (2006). Myelin Formation, Structure and Biochemistry. In: Siegel GJ, Agranoff BW, Albers RW, et al., editors. Basic Neurochemistry: Molecular, Cellular and Medical Aspects. 6th edition, Lippincott-Raven, Philadelphia. 
Rabesandratana, H., Toutant, J. P., Reggio, H. and Vidal, M. (1998). Decay-accelerating factor (CD55) and membrane inhibitor of reactive lysis (CD59) are released within exosomes during In vitro maturation of reticulocytes. Blood 91, 2573-2580.

Rajasekharan, S., Baker, K. A., Horn, K. E., Jarjour, A. A., Antel, J. P. and Kennedy, T. E. (2009). Netrin 1 and Dcc regulate oligodendrocyte process branching and membrane extension via Fyn and RhoA. Development 136, 415-426.

Rajendran, L., Honsho, M., Zahn, T. R., Keller, P., Geiger, K. D., Verkade, P. and Simons, K. (2006). Alzheimer's disease $\beta$-amyloid peptides are released in association with exosomes. Proceedings of the National Academy of Sciences of the United States of America 103, 1117211177.

Readhead, C., Schneider, A., Griffiths, I. and Nave, K. A. (1994). Premature arrest of myelin formation in transgenic mice with increased proteolipid protein gene dosage. Neuron 12, 583595 .

Reitsma, S., Slaaf, D. W., Vink, H., Van Zandvoort, M. A. M. J. and Oude Egbrink, M. G. A. (2007). The endothelial glycocalyx: composition, functions, and visualization. Pflugers Archiv $454,345-359$.

Relucio, J., Tzvetanova, I. D., Ao, W., Lindquist, S. and Colognato, H. (2009). Laminin alters fyn regulatory mechanisms and promotes oligodendrocyte development. Journal of Neuroscience 29, 11794-11806.

Richardson, W. D., Kessaris, N. and Pringle, N. (2006). Oligodendrocyte wars. Nature Reviews Neuroscience 7, 11-18.

Rosenberg, S. S., Powell, B. L. and Chan, J. R. (2007). Receiving mixed signals: uncoupling oligodendrocyte differentiation and myelination. Cellular and Molecular Life Sciences 64, 30593068.

Rosenbluth, J., Nave, K.-A., Mierzwa, A. and Schiff, R. (2006). Subtle myelin defects in PLP-null mice. Glia 54, 172-182.

Rutishauser, U. (2008). Polysialic acid in the plasticity of the developing and adult vertebrate nervous system. Nature Reviews Neuroscience 9, 26-35.

Rutishauser, U., Acheson, A., Hall, A. K., Mann, D. M. and Sunshine, J. (1990). The neural cell adhesion molecule (NCAM) as a regulator of cell-cell interactions. Science 240,53-57.

Sabri, S., Pierres, A., Benoliel, A. and Bongrand, P. (1995). Influence of surface charges on cell adhesion: difference between static and dynamic conditions. Biochem Cell Biol 73, 411-20. 
Sabri, S., Soler, M., Foa, C., Pierres, A., Benoliel, A. and Bongrand, P. (2000). Glycocalyx modulation is a physiological means of regulating cell adhesion. Journal of Cell Science 113, $1589-1600$.

Sackmann, E. and Bruinsma, R. F. (2002). Cell adhesion as wetting transition? Chemphyschem 3, 262-9.

Saito, M. and Yu, R. K. (1986). Further characterization of a myelin-associated neuraminidase: properties and substrate specificity. Journal of Neurochemistry 47, 632-641.

Saito, M. and Yu, R. K. (1993). Possible role of myelin-associated neuraminidase in membrane adhesion. Journal of Neuroscience Research 36, 127-132.

Schafer, D. P., Bansal, R., Hedstrom, K. L., Pfeiffer, S. E. and Rasband, M. N. (2004). Does paranode formation and maintenance require partitioning of neurofascin 155 into lipid rafts? Journal of Neuroscience 24, 3176-3185.

Schwarzmann, G., Hofmann, P., Pütz, U. and Albrecht, B. (1995). Demonstration of direct glycosylation of nondegradable glucosylceramide analogs in cultured cells. The Journal of Biological Chemistry 270, 21271-21276.

Sherman, D. L. and Brophy, P. J. (2005). Mechanisms of axon ensheathment and myelin growth. Nature Reviews Neuroscience 6, 683-690.

Simons, M., Friedrichson, T., Schulz, J. B., Pitto, M., Masserini, M. and Kurzchalia, T. V. (1999). Exogenous administration of gangliosides displaces GPI-anchored proteins from lipid microdomains in living cells. Molecular Biology of the Cell 10, 3187-96.

Simons, M. and Raposo, G. (2009). Exosomes - vesicular carriers for intercellular communication. Current Opinion in Cell Biology 21, 575-581.

Simons, M. and Trajkovic, K. (2006). Neuron-glia communication in the control of oligodendrocyte function and myelin biogenesis. Journal of Cell Science 119, 4381-4389.

Simons, M. and Trotter, J. (2007). Wrapping it up: the cell biology of myelination. Current Opinion in Neurobiology 17, 533-540.

Skog, J., Würdinger, T., Van Rijn, S., Meijer, D. H., Gainche, L., Sena-Esteves, M., Curry, W. T., Carter, B. S., Krichevsky, A. M. and Breakefield, X. O. (2008). Glioblastoma microvesicles transport RNA and proteins that promote tumour growth and provide diagnostic biomarkers. Nature Cell Biology 10, 1470-1476.

Somasundaram, R. and Herlyn, M. (2012). Melanoma exosomes: messengers of metastasis. Nature Medicine 18, 853-854. 
Sommer, I. and Schachner, M. (1981). Monoclonal antibodies (O1 to O4) to oligodendrocyte cell surfaces: an immunocytological study in the central nervous system. Developmental Biology 83, 311-27.

Sospedra, M. and Martin, R. (2005). Immunology of multiple sclerosis. Review Literature and Arts of The Americas 23, 683-747.

Sperber, B. R., Boyle-Walsh, E. A., Engleka, M. J., Gadue, P., Peterson, A. C., Stein, P. L., Scherer, S. S. and McMorris, F. A. (2001). A unique role for Fyn in CNS myelination. Journal of Neuroscience 21, 2039-2047.

Stevens, B., Porta, S., Haak, L. L., Gallo, V. and Fields, R. D. (2002). Adenosine: a neuron-glial transmitter promoting myelination in the CNS in response to action potentials. Neuron 36, 855-868.

Stoykova, L. I., Beesley, J. S., Grinspan, J. B. and Glick, M. C. (2001). ST8Sia IV mRNA corresponds with the biosynthesis of alpha2,8sialyl polymers but not oligomers in rat oligodendrocytes. Journal of Neuroscience Research 66, 497-505.

Strilić, B., Eglinger, J., Krieg, M., Zeeb, M., Axnick, J., Babál, P., Müller, D. J. and Lammert, E. (2010). Electrostatic cell-surface repulsion initiates lumen formation in developing blood vessels. Current Biology 20, 2003-2009.

Stritt, C., Stern, S., Harting, K., Manke, T., Sinske, D., Schwarz, H., Vingron, M., Nordheim, A. and Knöll, B. (2009). Paracrine control of oligodendrocyte differentiation by SRF-directed neuronal gene expression. Nature Neuroscience 12, 418-427.

Subra, C., Laulagnier, K., Perret, B. and Record, M. (2007). Exosome lipidomics unravels lipid sorting at the level of multivesicular bodies. Biochimie 89, 205-212.

Tarbell, J. M. and Pahakis, M. Y. (2006). Mechanotransduction and the glycocalyx. Journal of Internal Medicine 259, 339-350.

Taylor, M. E. and Drickamer, K. (2007). Paradigms for glycan-binding receptors in cell adhesion. Current Opinion in Cell Biology 19, 572-577.

Temple, S. and Raff, M. C. (1986). Clonal analysis of oligodendrocyte development in culture: evidence for a developmental clock that counts cell divisions. Cell 44, 773-779.

Ter Beest, M. B., Hoekstra, K., Sein, A. and Hoekstra, D. (1994). Reconstitution of proteolipid protein: some properties and its role in interlamellar attachment. The Biochemical Journal 300 ( Pt 2, 545-552.

Théry, C. (2011). Exosomes: secreted vesicles and intercellular communications. F1000 Biology Reports 3, 15 . 
Thomas, J. L., Spassky, N., Perez Villegas, E. M., Olivier, C., Cobos, I., Goujet-Zalc, C., Martínez, S. and Zalc, B. (2000). Spatiotemporal development of oligodendrocytes in the embryonic brain. Journal of Neuroscience Research 59, 471-476.

Trajkovic, K., Dhaunchak, A. S., Goncalves, J. T., Wenzel, D., Schneider, A., Bunt, G., Nave, K.-A. and Simons, M. (2006). Neuron to glia signaling triggers myelin membrane exocytosis from endosomal storage sites. The Journal of Cell Biology 172, 937-948.

Trajkovic, K., Hsu, C., Chiantia, S., Rajendran, L., Wenzel, D., Wieland, F., Schwille, P., Brügger, B. and Simons, M. (2008). Ceramide triggers budding of exosome vesicles into multivesicular endosomes. Science 319, 1244-1247.

Trapp, B. D., Nishiyama, A., Cheng, D. and Macklin, W. (1997). Differentiation and death of premyelinating oligodendrocytes in developing rodent brain. The Journal of Cell Biology 137, $459-468$.

Trotter, J., Bitter-Suermann, D. and Schachner, M. (1989). Differentiation-regulated loss of the polysialylated embryonic form and expression of the different polypeptides of the neural cell adhesion molecule by cultured oligodendrocytes and myelin. Journal of Neuroscience Research 22, 369-383.

Tyler, W. A., Gangoli, N., Gokina, P., Kim, H. A., Covey, M., Levison, S. W. and Wood, T. L. (2009). Activation of the mammalian target of rapamycin (mTOR) is essential for oligodendrocyte differentiation. Journal of Neuroscience 29, 6367-6378.

Umemori, H., Sato, S., Yagi, T., Aizawa, S. and Yamamoto, T. (1994). Initial events of myelination involve Fyn tyrosine kinase signalling. Nature $367,572-576$.

Valadi, H., Ekström, K., Bossios, A., Sjöstrand, M., Lee, J. J. and Lötvall, J. O. (2007). Exosomemediated transfer of mRNAs and microRNAs is a novel mechanism of genetic exchange between cells. Nature Cell Biology 9,654-659.

Van Der Goot, F. G. and Gruenberg, J. (2006). Intra-endosomal membrane traffic. Trends in Cell Biology 16, 514-521.

Vella, L. J., Sharples, R. A., Lawson, V. A., Masters, C. L., Cappai, R. and Hill, A. F. (2007). Packaging of prions into exosomes is associated with a novel pathway of $\mathrm{PrP}$ processing. The Journal of Pathology 211, 582-590.

Vella, L. J., Sharples, R. A., Nisbet, R. M., Cappai, R. and Hill, A. F. (2008). The role of exosomes in the processing of proteins associated with neurodegenerative diseases. European Biophysics Journal 37, 323-332. 
Vitte, J., Benoliel, A.-M., Pierres, A. and Bongrand, P. (2005). Regulation of cell adhesion. Clinical Hemorheology and Microcirculation 33, 167-188.

Wang, S., Sdrulla, A. D., DiSibio, G., Bush, G., Nofziger, D., Hicks, C., Weinmaster, G. and Barres, B. A. (1998). Notch receptor activation inhibits oligodendrocyte differentiation. Neuron 21, 63-75.

Weimbs, T. and Stoffel, W. (1992). Proteolipid protein (PLP) of CNS myelin: positions of free, disulfide-bonded, and fatty acid thioester-linked cysteine residues and implications for the membrane topology of PLP. Biochemistry 31, 12289-12296.

Weinbaum, S., Tarbell, J. M. and Damiano, E. R. (2007). The structure and function of the endothelial glycocalyx layer. Annual Review of Biomedical Engineering 9, 121-167.

White, R., Gonsior, C., Bauer, N. M., Kraemer-Albers, E. M., Luhmann, H. J. and Trotter, J. (2011). HnRNP F is a novel component of oligodendroglial RNA transport granules contributing to the regulation of MBP protein synthesis. The Journal of Biological Chemistry 287, 1742-1754.

White, R., Gonsior, C., Krämer-Albers, E.-M., Stöhr, N., Hüttelmaier, S. and Trotter, J. (2008). Activation of oligodendroglial Fyn kinase enhances translation of mRNAs transported in hnRNP A2-dependent RNA granules. The Journal of Cell Biology 181, 579-586.

Wilkins, A., Majed, H., Layfield, R., Compston, A. and Chandran, S. (2003). Oligodendrocytes promote neuronal survival and axonal length by distinct intracellular mechanisms: a novel role for oligodendrocyte-derived glial cell line-derived neurotrophic factor. Journal of Neuroscience 23, 4967-4974.

Williams, K. A. and Deber, C. M. (1993). The structure and function of central nervous system myelin. Critical Reviews in Clinical Laboratory Sciences 30, 29-64.

Willison, H. J. (2005). The immunobiology of Guillain-Barré syndromes. Journal of the Peripheral Nervous System 10, 94-112.

Ye, F., Chen, Y., Hoang, T., Montgomery, R. L., Zhao, X.-h., Bu, H., Hu, T., Taketo, M. M., Van Es, J. H., Clevers, H., Hsieh, J., Bassel-Duby, R., Olson, E. N. and Lu, Q. R. (2009). HDAC1 and HDAC2 regulate oligodendrocyte differentiation by disrupting the beta-catenin-TCF interaction. Nature Neuroscience 12, 829-838.

Yu, R. K., Macala, L. J., Farooq, M., Sbaschnig-Agler, M., Norton, W. T. and Ledeen, R. W. (1989). Ganglioside and lipid composition of bulk-isolated rat and bovine oligodendroglia. Journal of Neuroscience Research 23, 136-141. 
Zhao, X., He, X., Han, X., Yu, Y., Ye, F., Chen, Y., Hoang, T., Xu, X., Mi, Q.-S., Xin, M., Wang, F., Appel, B. and Lu, Q. R. (2010). MicroRNA-mediated control of oligodendrocyte differentiation. Neuron 65, 612-626. 


\title{
Curriculum Vitae
}

\author{
Mostafa Bakhti \\ born on $11^{\text {th }}$ September 1982 in Dehloran, Iran \\ Hermann-Rein-Str. 3, 37075, Göttingen, Germany \\ Phone +49 (0) 5513899534 \\ E-Mail bakhti@em.mpg.de
}

\begin{abstract}
Education
2008 - present Ph.D. candidate, Max Planck Institute for Experimental Medicine, Göttingen, Germany

2007 Research assistant, Molecular Biology Unit, Pasteur Institute, Tehran, Iran

2004 - 2006 M.Sc. Degree of Science in Cellular and Molecular Biology, School of Biology, University College of Science, University of Tehran, Iran

2000 - 2004 Bachelors of Science in Biology, Department of Biology, Faculty of Science, University of Isfahan, Iran
\end{abstract}

\section{Honors \& Achievements}

2006 - Present Membership of Iranian talented youth

2004-2006 Iranian Agriculture Bank fellowship for being selected as excellent student.

2004 Ranked $2^{\text {nd }}$ and achieved silver medal in the final stage of the National Olympiad for undergraduate students, administered by the National Education Measurement Organization.

2004

Ranked $1^{\text {st }}$ in Cellular and Molecular Biology among up to 7200 participants in the national exam for entering nationwide M.Sc. programs in biological science.

\section{Publications}

Yurlova L, Kahya N, Aggarwal S, Kaiser H-S, Chiantia S, Bakhti M, Pewzner-Jung Y, Ben-David O, Futerman A H., Brügger B and Simons M. (2011) Self-Segregation of Myelin Membrane Lipids in Model Membranes. Biophys. J. 101 (11):2713-2720

Fitzner D, Schnaars M, van Rossum D, Krishnamoorthy G, Dibaj P, Bakhti M, Regen T, Hanisch UK, Simons M. (2011) Selective transfer of exosomes from oligodendrocytes to microglia by macropinocytosis. J Cell Sci. 124 (Pt 3):447-58

Bakhti M, Winter C, Simons M. (2011) Inhibition of myelin membrane sheath formation by oligodendrocyte-derived exosome-like vesicles. J Biol Chem. 286 (1):787-96 
Hsu C, Morohashi Y, Yoshimura S, Manrique-Hoyos N, Jung S, Lauterbach MA, Bakhti M, Grønborg M, Möbius W, Rhee J, Barr FA, Simons M. (2010) Regulation of exosome secretion by Rab35 and its GTPase-activating proteins TBC1D10A-C. J Cell Biol. 189:223-32

Khazaei MR, Bakhti M, Habibi-Rezaei M. (2010) Nicotine reduces the cytotoxic effect of glycated proteins on microglial cells. Neurochem. Res. 35:548-58

M.R. Khazaei, M. Habibi-Rezaei, A.A. Moosavi-Movahedi, A.A. Sarrafnejhad, F. Sabouni, M. Bakhti, (2008) Microglial cell death induced by glycated bovine serum albumin: Nitric oxide involvement. J.Biochem 144:197-206

M. Bakhti, M. Habibi-Rezaei, A.A. Moosavi Movahedi, M.R. Khazaei. (2007) Consequential Alterations in Hemoglobin Structure upon Glycation with Fructose: Prevention by Acetylsalicylic Acid. J.Biochem 141:827-833 